\title{
Formal Gevrey Theory for Singular First Order Quasi-Linear Partial Differential Equations
}

By

\author{
Masaki HiBINO*
}

\begin{abstract}
This paper is concerned with the existence, the uniqueness, convergence and divergence of formal power series solutions of singular first order quasi-linear partial differential equations. Firstly we give the condition under which the formal solution exists uniquely. However, this formal power series solution does not necessarily converge. So we characterize the rate of divergence of the formal solution via the Gevrey order of formal power series. The Gevrey orders of formal solutions are determined by the Newton polyhedrons of nonlinear partial differential operators.
\end{abstract}

\section{$\S 1 . \quad$ Introduction and Main Result}

In this paper we are concerned with formal power series solutions of the following first order quasi-linear partial differential equation:

$$
\begin{gathered}
P u(x) \equiv \sum_{i=1}^{d} a_{i}(x, u(x)) D_{i} u(x)=f(x, u(x)), \quad u(0)=0, \\
x=\left(x_{1}, \ldots, x_{d}\right) \in \mathbf{C}^{d}, u \in \mathbf{C}, \quad D_{i}=\partial_{x_{i}}=\frac{\partial}{\partial x_{i}}
\end{gathered}
$$

where coefficients $a_{i}(x, u)(i=1, \ldots, d)$ and $f(x, u)$ are holomorphic in a neighborhood of $(x, u)=(0,0)$.

Communicated by T. Kawai. Recieved October 1, 2004. Revised May 30, 2005, September 5, 2005.

2000 Mathematics Subject Classification(s): Primary 35C10; Secondary 35A10.

* Research Fellow of the Japan Society for the Promotion of Science

Department of Mathematics, Meijo University, Tempaku, Nagoya, Aichi 468-8502, Japan. Current address: Department of Intelligent Mechanical Engineering, Okayama University of Science, 1-1 Ridai-cho, Okayama, 700-0005, Japan e-mail: hibino@are.ous.ac.jp

(c) 2006 Research Institute for Mathematical Sciences, Kyoto University. All rights reserved. 
We shall study the case where

$$
a_{i}(0,0)=0 \text { for all } i=1, \ldots, d,
$$

which is called a singular or degenerate case. Moreover as a compatibility condition we assume the following:

$$
f(0,0)=0 .
$$

In the following we always assume (1.2) and (1.3). We remark that by (1.2) Cauchy-Kowalevsky's theorem is not available.

We have two purposes. The first one is to prove the existence and the uniqueness of the formal power series solution $u(x)=\sum_{|\alpha| \geq 1} u_{\alpha} x^{\alpha}(\alpha=$ $\left.\left(\alpha_{1}, \ldots, \alpha_{d}\right) \in \mathbf{N}^{d}, \mathbf{N}=\{0,1,2, \ldots\},|\alpha|=\alpha_{1}+\cdots+\alpha_{d}, x^{\alpha}=x_{1}{ }^{\alpha_{1}} \cdots x_{d}{ }^{\alpha_{d}}\right)$ centered at the origin for the singular equation (1.1). As we will see later, we can prove it under some condition on the Jacobi matrix at the origin of the vector field $\mathbf{C}^{d} \ni x \mapsto\left(a_{1}(x, 0), \ldots, a_{d}(x, 0)\right) \in \mathbf{C}^{d}$. However, this formal power series solution $u(x)$ does not necessarily converge. So we would like to obtain the rate of divergence, which is called the Gevrey order, of the formal solution (cf. Definition 1.1). This is the second purpose of this paper. Hibino [2][3] studied the same problems for linear equations and semi-linear equations. In this paper we generalize these studies to quasi-linear equations.

The content of this paper is as follows: In $\S 1.1$ we introduce the result in [3] for semi-linear equations. To state the result (Theorem 1.1), we give the definition of the Gevrey order and explain the Poincaré condition, which assures the existence and the uniqueness of the formal solution. In $\S 1.2$, using the notation provided in $\S 1.1$, we give the main result in this paper (Theorem 1.2). Under one additional condition (cf. (1.6)), we will obtain the same result as that of [3]. In $\S 1.3$ we introduce literature studying related topics. In $\S 2$ we divide equations into seven classes, and for each class we give a precise Gevrey order of the formal solution, which is determined by the Newton Polyhedron of a quasi-linear differential operator (Theorem 2.1). Theorem 1.2 is obtained as a corollaly of Theorem 2.1. The proof of Theorem 2.1 is done through $\S 4$, $\S 5$ and $\S 6$ by using the contraction mapping principle in Banach spaces which consist of formal power series. The Banach spaces employed in the proof will be introduced in $\S 3$.

\section{§1.1. Result for Semi-Linear Equations}

In this subsection we state the theorem obtained in [3] for semi-linear equations. First we give the definition of the Gevrey order, which gives the rate of divergence of formal power series. 
Definition 1.1. Let $u(x)=\sum_{\alpha \in \mathbf{N}^{d}} u_{\alpha} x^{\alpha}$ be a formal power series centered at the origin. We say that $u(x)$ is of Gevrey- $\{s\}$ class $\left(s=\left(s_{1}, \ldots, s_{d}\right) \in\right.$ $\mathbf{R}^{d}$ ) if the power series

$$
\mathcal{B}_{s}[u](\xi) \equiv \sum_{\alpha \in \mathbf{N}^{d}} u_{\alpha} \frac{\xi^{\alpha}}{(\alpha !)^{s-1^{(d)}}}
$$

converges in a neighborhood of $\xi=0$, where $1^{(d)}=(\overbrace{1, \ldots, 1}^{d}), s-1^{(d)}=\left(s_{1}-\right.$ $\left.1, \ldots, s_{d}-1\right)$ and $(\alpha !)^{s-1^{(d)}}=\left(\alpha_{1} !\right)^{s_{1}-1} \cdots\left(\alpha_{d} !\right)^{s_{d}-1} . G^{\{s\}}$ denotes the set of all formal power series being of Gevrey- $\{s\}$ class. In particular, $u(x) \in G^{\left\{1^{(d)}\right\}}$ if and only if $u(x)$ is a convergent power series near $x=0$.

Now let us consider the following singular first order semi-linear partial differential equation:

$$
\widetilde{P} u(x) \equiv \sum_{i=1}^{d} a_{i}(x) D_{i} u(x)=f(x, u(x)), \quad u(0)=0,
$$

where coefficients $a_{i}(x)(i=1, \ldots, d)$ and $f(x, u)$ are holomorphic in a neighborhood of $x=0$ and $(x, u)=(0,0)$, respectively. Moreover it is assumed that $a_{i}(0)=0$ for all $i=1, \ldots, d$ and (1.3).

Let $D_{x} a(0)=\left(D_{j} a_{i}(0)\right)_{i, j=1, \ldots, d}$ be the Jacobi matrix at the origin of the vector field $x \mapsto\left(a_{1}(x), \ldots, a_{d}(x)\right)$ and let its Jordan canonical form be

$$
\left(\begin{array}{lllll}
A & & & \\
& B_{1} & & & \\
& & \ddots & & \\
& & & B_{k} & \\
& & & & O_{p}
\end{array}\right)
$$

where

$$
A=\left(\begin{array}{cccc}
\lambda_{1} & \delta_{1} & & \\
& \lambda_{2} & \ddots & \\
& & \ddots & \delta_{m-1} \\
& & & \lambda_{m}
\end{array}\right), B_{h}=\underbrace{\left(\begin{array}{cccc}
0 & 1 & \\
& 0 & \ddots & \\
& & \ddots & 1 \\
& & & 0
\end{array}\right)}_{n_{h}}, \begin{aligned}
& \lambda_{i} \neq 0(i=1, \ldots, m), \\
& \delta_{i}=0 \text { or } 1 \\
& (i=1, \ldots, m-1), \\
& h=1, \ldots, k,
\end{aligned}
$$

and $O_{p}$ is a zero-matrix of order $p\left(m, k, p \geq 0 ; n_{h} \geq 2 ; m+n_{1}+\cdots+n_{k}+p=d\right)$. 
Let us assume the following condition (Po) according to the value of $m$ ( "Po" derives from Poincaré):

$$
\text { (Po) } \begin{cases}\left|\sum_{i=1}^{m} \lambda_{i} \alpha_{i}-f_{u}(0,0)\right|>c|\alpha| \text { for all } \alpha \in \mathbf{N}^{m} & \text { (if } m \geq 1), \\ f_{u}(0,0) \neq 0 & \text { (if } m=0),\end{cases}
$$

where $f_{u}(0,0)=(\partial f / \partial u)(0,0)$, and $c$ is a positive constant independent of $\alpha \in \mathbf{N}^{m}$.

The main result in [3] is stated as follows:

Theorem 1.1 ([3]). Under the condition (Po), the equation (1.4) has a unique formal power series solution $u(x)=\sum_{|\alpha| \geq 1} u_{\alpha} x^{\alpha}$. Furthermore $u(x) \in$ $G^{\{2 N, \ldots, 2 N\}}$, where

$$
N= \begin{cases}\max \left\{n_{1}, \ldots, n_{k}\right\} & (\text { if } k \geq 1) \\ 1 & (\text { if } k=0 \text { and } p \geq 1) \\ \frac{1}{2} & (\text { if } k=p=0)\end{cases}
$$

Therefore in the case $k=p=0$ the formal solution converges. In other cases it diverges in general.

As was mentioned before, the purpose of this paper is to generalize the above result to quasi-linear equations.

Remark 1.1. The estimates of the Gevrey order given in Theorem 1.1 could be improved if the coefficients $a_{i}(x)$ have a higher order root for $x=$ 0 . For example, in the case $m=k=0$ and $p \geq 1$, the formal solution $u(x)=\sum_{n=1}^{\infty}(2 n-3) ! ! x^{2 n-1}((-1) ! !=1)$ of the ordinary differential equation $-x^{3}(d / d x) u(x)+u(x)=x$ belongs to $G^{\{3 / 2\}}$. Such a precise Gevrey order will be given in Theorem 2.1 .

\section{§1.2. Main Result}

Let us state the main result in this paper.

Let $J$ be the Jacobi matrix at the origin of the vector field $x \mapsto\left(a_{1}(x, 0), \ldots\right.$, $\left.a_{d}(x, 0)\right)$, that is, $J=\left(D_{j} a_{i}(0,0)\right)_{i, j=1, \ldots, d}$, and let us write its Jordan canonical form as (1.5).

Similarly to Theorem 1.1, we assume the condition ( $\mathrm{Po}$ ). Moreover we assume the following additional condition:

$$
\frac{\partial a_{i}}{\partial u}(0,0)=0 \text { for all } i=1, \ldots, d .
$$


Now the main result in this paper is stated as follows:

Theorem 1.2. $\quad$ Under the conditions (Po) and (1.6), the equation (1.1) has a unique formal power series solution $u(x)=\sum_{|\alpha| \geq 1} u_{\alpha} x^{\alpha}$. Furthermore $u(x) \in G^{\{2 N, \ldots, 2 N\}}$, where $N$ is same as in Theorem 1.1 .

In order to prove Theorem 1.2, as a first step we shall transform the equation (1.1) in the next section. For that transformed equation we can obtain the precise Gevrey order in individual variables of the formal solution (Theorem 2.1). We prove the unique existence of the formal solution and its Gevrey order separately. However, the proof of the unique existence is quite similar to that for semi-linear equations (cf. $\S 6$ in [3]), so in this paper we omit it. Admitting the unique existence of the formal solution, we will prove its Gevrey order in $\S 4$ (the case $m \neq 0$ and $k=p=0$ ), $\S 5$ (the case $m=0$ ) and $\S 6$ (the case $m \neq 0$ and $(k, p) \neq(0,0))$ by adopting the contraction mapping principle in Banach spaces introduced in $\S 3$. This route of the proof is same as that in [3], but the Banach spaces employed in the proof is slightly different from those used in [3] (cf. Definition 3.1 and Remark 3.1).

\section{§1.3. Some Remarks on Related Topics}

The studies in this paper and [2][3] are inspired by the study in Ōshima [9]. He studied a characterization of the kernel and the cokernel of the linear mapping

$$
\begin{gathered}
P_{0}: \mathcal{O} \rightarrow \mathcal{O} \\
\left(P_{0} u(x)=\sum_{i=1}^{d} a_{i}(x) D_{i} u(x)+b(x) u(x)\right),
\end{gathered}
$$

where $\mathcal{O}$ is the set of holomorphic functions at the origin, and $a_{i}(x), b(x) \in \mathcal{O}$. He studied the case $m \geq 1$ and $k=0$ in our notation, and obtained the condition under which the formal solution converges. That condition is called the simple ideal condition (cf. Remark 2.2). However, as mentioned in our theorem, when $m \geq 1, k=0$ and $p \geq 1$, if we remove the simple ideal condition, the formal solution diverges in general and it belongs to $G^{\{2, \ldots, 2\}}$.

The problem of convergence and divergence for formal solutions has been studied by many mathematicians. Higher order equations are studied by Miyake [5], Miyake-Hashimoto [6] and Yamazawa [13]. Nonlinear equations are studied in Gérard-Tahara [1], Miyake-Shirai [7] and Öuchi [10]. In Ouchi [10], the existence of asymptotic solutions is also dealt with. The study of Yamazawa [12] 
for semi-linear equations gives a different viewpoint from the author's study [3]. Moreover for linear equations, Kashiwara-Kawai-Sjöstrand [4] and MiyakeYoshino [8] give different characterizations of convergence of formal solutions. We can consider these studies to be the generalizations of Ōshima's study. However, in these studies the case $k \geq 1$ has not been studied. In this sense, [2][3] and Theorem 1.2 give a different type of generalization of Öshima [9].

Recently, Sibuya [11] studied greatly general higher order nonlinear ordinary differential equations with one parameter:

$$
F\left(x, u, \frac{d u}{d x}, \ldots, \frac{d^{l} u}{d x^{l}}, \varepsilon\right)=0,
$$

where $x, \varepsilon \in \mathbf{C}$. [11] assumed the existence of formal solutions which take the forms of

$$
u(x, \varepsilon)=\sum u_{n}(x) \varepsilon^{n},
$$

where $u_{n}(x)$ are holomorphic in a common domain, and gave the Gevrey orders of $u$ with respect to the parameter $\varepsilon$. Our equation (1.1) becomes a first order ordinary differential equation depending upon several parameters if all but one coefficient is identically zero. However, in this case our result (in case of one parameter) does not follow from that of [11]. In general, formal solutions can not be written in the forms of (1.7).

\section{$\S 2 . \quad$ Reduction of Equation and Newton Polyhedron}

In order to prove Theorem 1.2 we shall transform the equation (1.1) by a linear transform of independent variables which reduces the Jacobi matrix $J$ to its Jordan canonical form. By (1.2), (1.3) and (1.6), a reduced equation is written as follows according to the values of $m, k$ and $p$ :

Case (i) $m \geq 1, k \geq 1, p \geq 1$ :

$$
\begin{aligned}
& \Lambda u(x, y, z)=\left(P^{\prime}+P^{\prime \prime}+P^{\prime \prime \prime}+P^{\prime \prime \prime \prime}\right) u(x, y, z) \\
& +g_{0}(x, y, z)+g(x, y, z, u(x, y, z)), \\
& u(0,0,0)=0, \\
& x=\left(x_{1}, \ldots, x_{m}\right) \in \mathbf{C}^{m}, y=\left(y^{1}, \ldots, y^{k}\right) \in \mathbf{C}^{n_{1}+\cdots+n_{k}}, \\
& y^{h}=\left(y_{1}^{h}, \ldots, y_{n_{h}}^{h}\right) \in \mathbf{C}^{n_{h}}(h=1, \ldots, k), \\
& z=\left(z_{1}, \ldots, z_{p}\right) \in \mathbf{C}^{p} \text {, }
\end{aligned}
$$

where

$$
\Lambda=\sum_{i=1}^{m} \lambda_{i} x_{i} \frac{\partial}{\partial x_{i}}-f_{u}(0,0),
$$


and

$$
\begin{aligned}
& P^{\prime} u=-\sum_{i=1}^{m-1} \delta_{i} x_{i+1} \frac{\partial u}{\partial x_{i}}+\sum_{i=1}^{m}\left(\sum_{\substack{|\alpha|+|\beta|+|\gamma|+r \geq 2 \\
|\alpha| \geq 1}}^{\text {finite }} c_{i \alpha \beta \gamma r}(x, y, z, u) x^{\alpha} y^{\beta} z^{\gamma} u^{r}\right) \frac{\partial u}{\partial x_{i}}, \\
& P^{\prime \prime} u=\sum_{h=1}^{k} \sum_{j_{h}=1}^{n_{h}}\left(\sum_{\substack{|\alpha|+|\beta|+|\gamma|+r \geq 2 \\
|\alpha| \geq 1}}^{\text {finite }} d_{j_{h} \alpha \beta \gamma r}^{h}(x, y, z, u) x^{\alpha} y^{\beta} z^{\gamma} u^{r}\right) \frac{\partial u}{\partial y^{h}{ }_{j_{h}}} \\
& +\sum_{q=1}^{p}\left(\sum_{\substack{|\alpha|+|\beta|+|\gamma|+r \geq 2 \\
|\alpha| \geq 1}}^{\text {finite }} e_{q \alpha \beta \gamma r}(x, y, z, u) x^{\alpha} y^{\beta} z^{\gamma} u^{r}\right) \frac{\partial u}{\partial z_{q}} \\
& P^{\prime \prime \prime} u=-\sum_{h=1}^{k} \sum_{j_{h}=1}^{n_{h}-1} y_{j_{h}+1}^{h} \frac{\partial u}{\partial y_{j_{h}}^{h}} \\
& +\sum_{h=1}^{k} \sum_{j_{h}=1}^{n_{h}}\left(\sum_{|\beta|+|\gamma|+r \geq 2}^{\text {finite }} d_{j_{h} \beta \gamma r}^{h}(x, y, z, u) y^{\beta} z^{\gamma} u^{r}\right) \frac{\partial u}{\partial y^{h}{ }_{j_{h}}} \\
& +\sum_{q=1}^{p}\left(\sum_{|\beta|+|\gamma|+r \geq 2}^{\text {finite }} e_{q \beta \gamma r}(x, y, z, u) y^{\beta} z^{\gamma} u^{r}\right) \frac{\partial u}{\partial z_{q}}, \\
& P^{\prime \prime \prime \prime} u=\sum_{i=1}^{m}\left(\sum_{|\beta|+|\gamma|+r \geq 2}^{\text {finite }} c_{i \beta \gamma r}(x, y, z, u) y^{\beta} z^{\gamma} u^{r}\right) \frac{\partial u}{\partial x_{i}} \\
& \left(x^{\alpha}=x_{1}{ }^{\alpha_{1}} \cdots x_{m}{ }^{\alpha_{m}}, y^{\beta}=\left(y^{1}\right)^{\beta^{1}} \cdots\left(y^{k}\right)^{\beta^{k}},\left(y^{h}\right)^{\beta^{h}}=\left(y^{h}{ }_{1}\right)^{\beta^{h}}{ }_{1} \ldots\left(y^{h}{ }_{n_{h}}\right)^{\beta^{h}{ }_{n_{h}}}\right. \\
& \left.(h=1, \ldots, k), z^{\gamma}=z_{1}^{\gamma_{1}} \cdots z_{p}^{\gamma_{p}}\right) . g_{0} \text { and } g \text { are holomorphic at the origin which }
\end{aligned}
$$

Case (ii) $m \geq 1, k \geq 1, p=0$ :

$$
\begin{aligned}
& \Lambda u(x, y)=\left(P^{\prime}+P^{\prime \prime}+P^{\prime \prime \prime}+P^{\prime \prime \prime \prime}\right) u(x, y)+g_{0}(x, y)+g(x, y, u(x, y)), \\
& u(0,0)=0
\end{aligned}
$$

where $g_{0}$ and $g$ are holomorphic at the origin which satisfy $g_{0}(0,0)=0$ and $g(x, y, 0) \equiv 0, g_{u}(0,0,0)=0$, respectively. The linear partial differential operator $\Lambda$ is same as (2.2), and

$$
P^{\prime} u=-\sum_{i=1}^{m-1} \delta_{i} x_{i+1} \frac{\partial u}{\partial x_{i}}+\sum_{i=1}^{m}\left(\sum_{\substack{|\alpha|+|\beta|+r \geq 2 \\|\alpha| \geq 1}}^{\text {finite }} c_{i \alpha \beta r}(x, y, u) x^{\alpha} y^{\beta} u^{r}\right) \frac{\partial u}{\partial x_{i}}
$$




$$
\begin{aligned}
P^{\prime \prime} u & =\sum_{h=1}^{k} \sum_{j_{h}=1}^{n_{h}}\left(\sum_{\substack{|\alpha|+\beta|+r \geq 2\\
| \alpha \mid \geq 1}}^{\text {finite }} d_{j_{h} \alpha \beta r}^{h}(x, y, u) x^{\alpha} y^{\beta} u^{r}\right) \frac{\partial u}{\partial y_{j_{h}}^{h}} \\
P^{\prime \prime \prime} u & =-\sum_{h=1}^{k} \sum_{j_{h}=1}^{n_{h}-1} y_{j_{h}+1}^{h} \frac{\partial u}{\partial y_{j_{h}}^{h}}+\sum_{h=1}^{k} \sum_{j_{h}=1}^{n_{h}}\left(\sum_{|\beta|+r \geq 2}^{\text {finite }} d_{j_{h} \beta r}^{h}(x, y, u) y^{\beta} u^{r}\right) \frac{\partial u}{\partial y_{j_{h}}{ }_{j_{h}}} \\
P^{\prime \prime \prime \prime} u & =\sum_{i=1}^{m}\left(\sum_{|\beta|+r \geq 2}^{\text {finite }} c_{i \beta r}(x, y, u) y^{\beta} u^{r}\right) \frac{\partial u}{\partial x_{i}}
\end{aligned}
$$

Case (iii) $m \geq 1, k=0, p \geq 1$ :

$$
\begin{aligned}
& \Lambda u(x, z)=\left(P^{\prime}+P^{\prime \prime}+P^{\prime \prime \prime}+P^{\prime \prime \prime \prime}\right) u(x, z)+g_{0}(x, z)+g(x, z, u(x, z)), \\
& u(0,0)=0
\end{aligned}
$$

where $g_{0}$ and $g$ are holomorphic at the origin with $g_{0}(0,0)=0$ and $g(x, z, 0) \equiv 0$, $g_{u}(0,0,0)=0$, respectively. The linear partial differential operator $\Lambda$ is same as $(2.2)$, and

$$
\begin{aligned}
& P^{\prime} u=-\sum_{i=1}^{m-1} \delta_{i} x_{i+1} \frac{\partial u}{\partial x_{i}}+\sum_{i=1}^{m}\left(\sum_{\substack{|\alpha|+|\gamma|+r \geq 2 \\
|\alpha| \geq 1}}^{\text {finite }} c_{i \alpha \gamma r}(x, z, u) x^{\alpha} z^{\gamma} u^{r}\right) \frac{\partial u}{\partial x_{i}}, \\
& P^{\prime \prime} u=\sum_{q=1}^{p}\left(\sum_{\substack{|\alpha|+|\gamma|+r \geq 2 \\
|\alpha| \geq 1}}^{\text {finite }} e_{q \alpha \gamma r}(x, z, u) x^{\alpha} z^{\gamma} u^{r}\right) \frac{\partial u}{\partial z_{q}} \\
& P^{\prime \prime \prime} u=\sum_{q=1}^{p}\left(\sum_{|\gamma|+r \geq 2}^{\text {finite }} e_{q \gamma r}(x, z, u) z^{\gamma} u^{r}\right) \frac{\partial u}{\partial z_{q}} \\
& P^{\prime \prime \prime \prime} u=\sum_{i=1}^{m}\left(\sum_{|\gamma|+r \geq 2}^{\text {finite }} c_{i \gamma r}(x, z, u) z^{\gamma} u^{r}\right) \frac{\partial u}{\partial x_{i}} .
\end{aligned}
$$

Case (iv) $m \geq 1, k=p=0$ :

$$
\begin{aligned}
\Lambda u(x)= & -\sum_{i=1}^{m-1} \delta_{i} x_{i+1} \frac{\partial u}{\partial x_{i}}(x)+\sum_{i=1}^{m}\left(\sum_{|\alpha|+r \geq 2}^{\text {finite }} c_{i \alpha r}(x, u(x)) x^{\alpha} u(x)^{r}\right) \frac{\partial u}{\partial x_{i}}(x) \\
& +g_{0}(x)+g(x, u(x)), \\
u(0)= & 0
\end{aligned}
$$

where $g_{0}$ and $g$ are holomorphic at the origin with $g_{0}(0)=0$ and $g(x, 0) \equiv 0$, $g_{u}(0,0)=0$, respectively. $\Lambda$ is same as $(2.2)$. 
Case (v) $m=0, k \geq 1, p \geq 1$ :

$$
f_{u}(0,0) \cdot u(y, z)=P^{\prime \prime \prime} u(y, z)+g_{0}(y, z)+g(y, z, u(y, z)), \quad u(0,0)=0,
$$

where $g_{0}$ and $g$ are holomorphic at the origin which satisfy $g_{0}(0,0)=0$ and $g(y, z, 0) \equiv 0, g_{u}(0,0,0)=0$, respectively, and

$$
\begin{aligned}
P^{\prime \prime \prime} u= & \sum_{h=1}^{k} \sum_{j_{h}=1}^{n_{h}-1} y_{j_{h}+1}^{h} \frac{\partial u}{\partial y^{h} j_{h}} \\
& +\sum_{h=1}^{k} \sum_{j_{h}=1}^{n_{h}}\left(\sum_{|\beta|+|\gamma|+r \geq 2}^{\text {finite }} d_{j_{h} \beta \gamma r}^{h}(y, z, u) y^{\beta} z^{\gamma} u^{r}\right) \frac{\partial u}{\partial y^{h}{ }_{j_{h}}} \\
& +\sum_{q=1}^{p}\left(\sum_{|\beta|+|\gamma|+r \geq 2}^{\text {finite }} e_{q \beta \gamma r}(y, z, u) y^{\beta} z^{\gamma} u^{r}\right) \frac{\partial u}{\partial z_{q}} .
\end{aligned}
$$

Case (vi) $m=0, k \geq 1, p=0$ :

$$
f_{u}(0,0) \cdot u(y)=P^{\prime \prime \prime} u(y)+g_{0}(y)+g(y, u(y)), \quad u(0)=0,
$$

where $g_{0}$ and $g$ are holomorphic at the origin which satisfy $g_{0}(0)=0$ and $g(y, 0) \equiv 0, g_{u}(0,0)=0$, respectively, and

$$
P^{\prime \prime \prime} u=\sum_{h=1}^{k} \sum_{j_{h}=1}^{n_{h}-1} y_{j_{h}+1}^{h} \frac{\partial u}{\partial y^{h}{ }_{j_{h}}}+\sum_{h=1}^{k} \sum_{j_{h}=1}^{n_{h}}\left(\sum_{|\beta|+r \geq 2}^{\text {finite }} d_{j_{h} \beta r}^{h}(y, u) y^{\beta} u^{r}\right) \frac{\partial u}{\partial y_{j_{h}}^{h}} .
$$

Case (vii) $m=k=0, p \geq 1$ :

$$
f_{u}(0,0) \cdot u(z)=P^{\prime \prime \prime} u(z)+g_{0}(z)+g(z, u(z)), \quad u(0)=0,
$$

where $g_{0}$ and $g$ are holomorphic at the origin with $g_{0}(0)=0$ and $g(z, 0) \equiv 0$, $g_{u}(0,0)=0$, respectively, and

$$
P^{\prime \prime \prime} u=\sum_{q=1}^{p}\left(\sum_{|\gamma|+r \geq 2}^{\text {finite }} e_{q \gamma r}(z, u) z^{\gamma} u^{r}\right) \frac{\partial u}{\partial z_{q}} .
$$

Now we shall study the equations (2.1), (2.3), (2.4), (2.5), (2.6), (2.7) and (2.8).

In order to give the Gevrey orders in an individual variable for formal solutions of the above equations, we study the Newton polyhedrons of nonlinear partial differential operators. 
Newton Polyhedron. Let

$$
L u(\xi)=\sum_{\alpha, \beta \in \mathbf{N}^{d},|\beta| \geq 1}^{\text {finite }} a_{\alpha \beta r}(\xi, u(\xi)) \xi^{\alpha} u(\xi)^{r} D_{\xi}^{\beta} u(\xi)
$$

$\left(\xi=\left(\xi_{1}, \ldots, \xi_{d}\right), D_{\xi}^{\beta}=\left(\partial / \partial \xi_{1}\right)^{\beta_{1}} \cdots\left(\partial / \partial \xi_{d}\right)^{\beta_{d}}\right)$ be a nonlinear partial differential operator which is linear with respect to derivatives, where all coefficients $a_{\alpha \beta r}(\xi, u)$ are holomorphic at the origin and do not vanish at the origin unless they vanish identically.

We define $Q(\alpha, \beta)\left(\subset \mathbf{R}^{d+1}\right)$ by

$$
\begin{aligned}
Q(\alpha, \beta)=\left\{(\mathcal{X}, \mathcal{W})=\left(\mathcal{X}_{1}, \ldots, \mathcal{X}_{d}, \mathcal{W}\right) \in\right. & \mathbf{R}^{d+1} ; \\
& \left.\mathcal{X}_{i} \geq \alpha_{i}-\beta_{i}(i=1, \ldots, d), \mathcal{W} \leq|\beta|\right\}
\end{aligned}
$$

and define $Q\left(\xi^{\alpha} u(\xi)^{r} D_{\xi}^{\beta} u(\xi)\right)$ by

$$
Q\left(\xi^{\alpha} u(\xi)^{r} D_{\xi}^{\beta} u(\xi)\right)=\bigcup_{i=1}^{d} Q\left(\alpha+r \mathrm{e}_{i}, \beta\right),
$$

where $\mathrm{e}_{i}=\left(\delta_{i 1}, \delta_{i 2}, \ldots, \delta_{i d}\right)\left(\delta_{i i^{\prime}}\right.$ : Kronecker's delta $)$. We remark that $Q\left(\xi^{\alpha} D_{\xi}^{\beta} u(\xi)\right)=Q(\alpha, \beta)$. Let us define the Newton polyhedron $N(L)$ of the operator $L$ by

$$
N(L)= \begin{cases}\operatorname{Ch}\left\{\bigcup_{(\alpha, \beta, r) \text { with } a_{\alpha \beta r} \neq 0} Q\left(\xi^{\alpha} u(\xi)^{r} D_{\xi}^{\beta} u(\xi)\right)\right\} & (\text { if } L \neq 0), \\ Q(0,0) & (\text { if } L=0),\end{cases}
$$

where Ch $A$ denotes the convex hull of a set $A \subset \mathbf{R}^{d+1}$.

Now we shall apply the above general definition to our differential operators $P^{\prime}+P^{\prime \prime}+P^{\prime \prime \prime}+P^{\prime \prime \prime \prime}$ (Cases (i), (ii) and (iii)) and $P^{\prime \prime \prime}$ (Cases (v), (vi) and (vii)). We remark that the correspondence of variables between $(x, y, z)$ and $\xi$ is given by

\begin{tabular}{|l|c|}
\hline & $\xi$ \\
\hline Case (i) & $(x, y, z)$ \\
Case (ii) & $(x, y)$ \\
Case (iii) & $(x, z)$ \\
Case (iv) & - \\
Case (v) & $(y, z)$ \\
Case (vi) & $y$ \\
Case (vii) & $z$ \\
\hline
\end{tabular}


In order to state the main theorem in this section, we shall define the sets $S_{i}$ $(i=1,2,3,5,6,7), \widetilde{S}_{j}, \widetilde{S}_{j}^{\prime}, \widetilde{S}_{j}^{\prime \prime}, S_{j}^{\prime}, S_{j}^{\prime \prime}(j=1,2,3)$ whose elements give the Gevrey orders of formal solutions.

Case (i) We define $\widetilde{\Pi}_{1}(\rho, \sigma, \tau)$ and $\Pi_{1}(\rho, \sigma, \tau)\left((\rho, \sigma, \tau) \in[1,+\infty)^{d}\right.$, $\rho=\left(\rho_{1}, \ldots, \rho_{m}\right) \in[1,+\infty)^{m}, \sigma=\left(\sigma^{1}, \ldots, \sigma^{k}\right) \in[1,+\infty)^{n_{1}+\cdots+n_{k}}, \sigma^{h}=$ $\left.\left(\sigma^{h}{ }_{1}, \ldots, \sigma^{h}{ }_{n_{h}}\right) \in[1,+\infty)^{n_{h}}, \tau=\left(\tau_{1}, \ldots, \tau_{p}\right) \in[1,+\infty)^{p}\right)$ by

$\widetilde{\Pi}_{1}(\rho, \sigma, \tau)=\left\{(\mathcal{X}, \mathcal{Y}, \mathcal{Z}, \mathcal{W}) \in \mathbf{R}^{d+1}\right.$

$$
\left.\left(\rho-1^{(m)}\right) \cdot \mathcal{X}+\left(\sigma-1^{\left(n_{1}+\cdots+n_{k}\right)}\right) \cdot \mathcal{Y}+\left(\tau-1^{(p)}\right) \cdot \mathcal{Z}-\mathcal{W} \geq-1\right\}
$$

$\left(\mathcal{X}=\left(\mathcal{X}_{1}, \ldots, \mathcal{X}_{m}\right), \mathcal{Y}=\left(\mathcal{Y}^{1}, \ldots, \mathcal{Y}^{k}\right), \mathcal{Y}^{h}=\left(\mathcal{Y}^{h}{ }_{1}, \ldots, \mathcal{Y}^{h}{ }_{n_{h}}\right)(h=1, \ldots, k)\right.$, $\mathcal{Z}=\left(\mathcal{Z}_{1}, \ldots, \mathcal{Z}_{p}\right) ; A \cdot B$ means the scalar product of $A$ and $\left.B\right)$ and

$$
\begin{aligned}
\Pi_{1}(\rho, \sigma, \tau)= & \left\{(\mathcal{X}, \mathcal{Y}, \mathcal{Z}, \mathcal{W}) \in \mathbf{R}^{d+1}\right. \\
& \left.\left(\rho-1^{(m)}\right) \cdot \mathcal{X}+\left(\sigma-1^{\left(n_{1}+\cdots+n_{k}\right)}\right) \cdot \mathcal{Y}+\left(\tau-1^{(p)}\right) \cdot \mathcal{Z}-\mathcal{W} \geq 0\right\},
\end{aligned}
$$

respectively, and define $\widetilde{S}_{1}, \widetilde{S}_{1}^{\prime}, \widetilde{S}_{1}^{\prime \prime}, S_{1}, S_{1}^{\prime}$, and $S_{1}^{\prime \prime}$ as follows:

$$
\begin{aligned}
& \widetilde{S}_{1}=\left\{(\rho, \sigma, \tau) \in[1,+\infty)^{d} ; N\left(P^{\prime}\right) \subset \widetilde{\Pi}_{1}(\rho, \sigma, \tau)\right\}, \\
& \widetilde{S}_{1}^{\prime}=\left\{(\rho, \sigma, \tau) \in[1,+\infty)^{d} ; N\left(P^{\prime \prime}\right) \subset \widetilde{\Pi}_{1}(\rho, \sigma, \tau)\right\}, \\
& \widetilde{S}_{1}^{\prime \prime}=\left\{(\rho, \sigma, \tau) \in[1,+\infty)^{d} ; N\left(P^{\prime \prime \prime \prime}\right) \subset \widetilde{\Pi}_{1}(\rho, \sigma, \tau)\right\}, \\
& S_{1}=\left\{(\rho, \sigma, \tau) \in[1,+\infty)^{d} ; N\left(P^{\prime \prime \prime}\right) \subset \Pi_{1}(\rho, \sigma, \tau)\right\}, \\
& S_{1}^{\prime}=\left\{(\rho, \sigma, \tau) \in[1,+\infty)^{d} ; N\left(P^{\prime \prime}\right) \subset \Pi_{1}(\rho, \sigma, \tau)\right\}, \\
& S_{1}^{\prime \prime}=\left\{(\rho, \sigma, \tau) \in[1,+\infty)^{d} ; N\left(P^{\prime \prime \prime \prime}\right) \subset \Pi_{1}(\rho, \sigma, \tau)\right\} .
\end{aligned}
$$

Case (ii) We define $\widetilde{\Pi}_{2}(\rho, \sigma)$ and $\Pi_{2}(\rho, \sigma)\left((\rho, \sigma) \in[1,+\infty)^{d}\right)$ by

$\widetilde{\Pi}_{2}(\rho, \sigma)=\left\{(\mathcal{X}, \mathcal{Y}, \mathcal{W}) \in \mathbf{R}^{d+1} ;\left(\rho-1^{(m)}\right) \cdot \mathcal{X}+\left(\sigma-1^{\left(n_{1}+\cdots+n_{k}\right)}\right) \cdot \mathcal{Y}-\mathcal{W} \geq-1\right\}$

and

$\Pi_{2}(\rho, \sigma)=\left\{(\mathcal{X}, \mathcal{Y}, \mathcal{W}) \in \mathbf{R}^{d+1} ;\left(\rho-1^{(m)}\right) \cdot \mathcal{X}+\left(\sigma-1^{\left(n_{1}+\cdots+n_{k}\right)}\right) \cdot \mathcal{Y}-\mathcal{W} \geq 0\right\}$,

respectively, and define $\widetilde{S}_{2}, \widetilde{S}_{2}^{\prime}, \widetilde{S}_{2}^{\prime \prime}, S_{2}, S_{2}^{\prime}$ and $S_{2}^{\prime \prime}$ as follows:

$$
\begin{aligned}
& \widetilde{S}_{2}=\left\{(\rho, \sigma) \in[1,+\infty)^{d} ; N\left(P^{\prime}\right) \subset \widetilde{\Pi}_{2}(\rho, \sigma)\right\}, \\
& \widetilde{S}_{2}^{\prime}=\left\{(\rho, \sigma) \in[1,+\infty)^{d} ; N\left(P^{\prime \prime}\right) \subset \widetilde{\Pi}_{2}(\rho, \sigma)\right\}, \\
& \widetilde{S}_{2}^{\prime \prime}=\left\{(\rho, \sigma) \in[1,+\infty)^{d} ; N\left(P^{\prime \prime \prime \prime}\right) \subset \widetilde{\Pi}_{2}(\rho, \sigma)\right\},
\end{aligned}
$$




$$
\begin{aligned}
& S_{2}=\left\{(\rho, \sigma) \in[1,+\infty)^{d} ; N\left(P^{\prime \prime \prime}\right) \subset \Pi_{2}(\rho, \sigma)\right\}, \\
& S_{2}^{\prime}=\left\{(\rho, \sigma) \in[1,+\infty)^{d} ; N\left(P^{\prime \prime}\right) \subset \Pi_{2}(\rho, \sigma)\right\}, \\
& S_{2}^{\prime \prime}=\left\{(\rho, \sigma) \in[1,+\infty)^{d} ; N\left(P^{\prime \prime \prime \prime}\right) \subset \Pi_{2}(\rho, \sigma)\right\} .
\end{aligned}
$$

Case (iii) We define $\widetilde{\Pi}_{3}(\rho, \tau)$ and $\Pi_{3}(\rho, \tau)\left((\rho, \tau) \in[1,+\infty)^{d}\right)$ by $\widetilde{\Pi}_{3}(\rho, \tau)=\left\{(\mathcal{X}, \mathcal{Z}, \mathcal{W}) \in \mathbf{R}^{d+1} ;\left(\rho-1^{(m)}\right) \cdot \mathcal{X}+\left(\tau-1^{(p)}\right) \cdot \mathcal{Z}-\mathcal{W} \geq-1\right\}$ and

$$
\Pi_{3}(\rho, \tau)=\left\{(\mathcal{X}, \mathcal{Z}, \mathcal{W}) \in \mathbf{R}^{d+1} ;\left(\rho-1^{(m)}\right) \cdot \mathcal{X}+\left(\tau-1^{(p)}\right) \cdot \mathcal{Z}-\mathcal{W} \geq 0\right\}
$$

respectively, and define $\widetilde{S}_{3}, \widetilde{S}_{3}^{\prime}, \widetilde{S}_{3}^{\prime \prime}, S_{3}, S_{3}^{\prime}, S_{3}^{\prime \prime}$ as follows:

$$
\begin{aligned}
& \widetilde{S}_{3}=\left\{(\rho, \tau) \in[1,+\infty)^{d} ; N\left(P^{\prime}\right) \subset \widetilde{\Pi}_{3}(\rho, \tau)\right\}, \\
& \widetilde{S}_{3}^{\prime}=\left\{(\rho, \tau) \in[1,+\infty)^{d} ; N\left(P^{\prime \prime}\right) \subset \widetilde{\Pi}_{3}(\rho, \tau)\right\}, \\
& \widetilde{S}_{3}^{\prime \prime}=\left\{(\rho, \tau) \in[1,+\infty)^{d} ; N\left(P^{\prime \prime \prime \prime}\right) \subset \widetilde{\Pi}_{3}(\rho, \tau)\right\}, \\
& S_{3}=\left\{(\rho, \tau) \in[1,+\infty)^{d} ; N\left(P^{\prime \prime \prime}\right) \subset \Pi_{3}(\rho, \tau)\right\}, \\
& S_{3}^{\prime}=\left\{(\rho, \tau) \in[1,+\infty)^{d} ; N\left(P^{\prime \prime}\right) \subset \Pi_{3}(\rho, \tau)\right\}, \\
& S_{3}^{\prime \prime}=\left\{(\rho, \tau) \in[1,+\infty)^{d} ; N\left(P^{\prime \prime \prime \prime}\right) \subset \Pi_{3}(\rho, \tau)\right\} .
\end{aligned}
$$

Case $(\mathbf{v})$ We define $\Pi_{5}(\sigma, \tau)\left((\sigma, \tau) \in[1,+\infty)^{d}\right)$ by $\Pi_{5}(\sigma, \tau)=\left\{(\mathcal{Y}, \mathcal{Z}, \mathcal{W}) \in \mathbf{R}^{d+1} ;\left(\sigma-1^{\left(n_{1}+\cdots+n_{k}\right)}\right) \cdot \mathcal{Y}+\left(\tau-1^{(p)}\right) \cdot \mathcal{Z}-\mathcal{W} \geq 0\right\}$ and define $S_{5}$ by

$$
S_{5}=\left\{(\sigma, \tau) \in[1,+\infty)^{d} ; N\left(P^{\prime \prime \prime}\right) \subset \Pi_{5}(\sigma, \tau)\right\} .
$$

Case (vi) We define $\Pi_{6}(\sigma)\left(\sigma \in[1,+\infty)^{d}\right)$ by

$$
\Pi_{6}(\sigma)=\left\{(\mathcal{Y}, \mathcal{W}) \in \mathbf{R}^{d+1} ;\left(\sigma-1^{\left(n_{1}+\cdots+n_{k}\right)}\right) \cdot \mathcal{Y}-\mathcal{W} \geq 0\right\}
$$

and define $S_{6}$ by

$$
S_{6}=\left\{\sigma \in[1,+\infty)^{d} ; N\left(P^{\prime \prime \prime}\right) \subset \Pi_{6}(\sigma, \tau)\right\} .
$$

Case (vii) We define $\Pi_{7}(\tau)\left(\tau \in[1,+\infty)^{d}\right)$ by

$$
\Pi_{7}(\tau)=\left\{(\mathcal{Z}, \mathcal{W}) \in \mathbf{R}^{d+1} ;\left(\tau-1^{(p)}\right) \cdot \mathcal{Z}-\mathcal{W} \geq 0\right\}
$$

and define $S_{7}$ by

$$
S_{7}=\left\{\tau \in[1,+\infty)^{d} ; N\left(P^{\prime \prime \prime}\right) \subset \Pi_{7}(\tau)\right\} .
$$

Then we obtain the following theorem. 
Theorem 2.1. In Case (i) (resp. (ii), (iii), (iv), (v), (vi) and (vii)), under the condition ( $\mathrm{Po}$ ) the equation (2.1) (resp. (2.3), (2.4), (2.5), (2.6), (2.7) and (2.8)) has a unique formal power series solution. Furthermore the formal solution belongs to $G^{\{s\}}$ if s satisfies the following condition:

Case (i) $P^{\prime \prime \prime \prime}=0 \Rightarrow s=(\rho, \sigma, \tau) \in \widetilde{S}_{1} \cap S_{1} \cap \widetilde{S}_{1}^{\prime}$,

$P^{\prime \prime}=0 \Rightarrow s=(\rho, \sigma, \tau) \in \widetilde{S}_{1} \cap S_{1} \cap \widetilde{S}_{1}^{\prime \prime}$,

$P^{\prime \prime}, P^{\prime \prime \prime \prime} \neq 0 \Rightarrow s=(\rho, \sigma, \tau) \in \widetilde{S}_{1} \cap S_{1} \cap\left\{\left(\widetilde{S}_{1}^{\prime} \cap S_{1}^{\prime \prime}\right) \cup\left(S_{1}^{\prime} \cap \widetilde{S}_{1}^{\prime \prime}\right)\right\} ;$

Case (ii) $P^{\prime \prime \prime \prime}=0 \Rightarrow s=(\rho, \sigma) \in \widetilde{S}_{2} \cap S_{2} \cap \widetilde{S}_{2}^{\prime}$,

$P^{\prime \prime}=0 \Rightarrow s=(\rho, \sigma) \in \widetilde{S}_{2} \cap S_{2} \cap \widetilde{S}_{2}^{\prime \prime}$,

$P^{\prime \prime}, P^{\prime \prime \prime \prime} \neq 0 \Rightarrow s=(\rho, \sigma) \in \widetilde{S}_{2} \cap S_{2} \cap\left\{\left(\widetilde{S}_{2}^{\prime} \cap S_{2}^{\prime \prime}\right) \cup\left(S_{2}^{\prime} \cap \widetilde{S}_{2}^{\prime \prime}\right)\right\} ;$

Case (iii) $\quad P^{\prime \prime \prime \prime}=0 \Rightarrow s=(\rho, \tau) \in \widetilde{S}_{3} \cap S_{3} \cap \widetilde{S}_{3}^{\prime}$,

$P^{\prime \prime}=0 \Rightarrow s=(\rho, \tau) \in \widetilde{S}_{3} \cap S_{3} \cap \widetilde{S}_{3}^{\prime \prime}$,

$P^{\prime \prime}, P^{\prime \prime \prime \prime} \neq 0 \Rightarrow s=(\rho, \tau) \in \widetilde{S}_{3} \cap S_{3} \cap\left\{\left(\widetilde{S}_{3}^{\prime} \cap S_{3}^{\prime \prime}\right) \cup\left(S_{3}^{\prime} \cap \widetilde{S}_{3}^{\prime \prime}\right)\right\} ;$

Case (iv) $s=1^{(d)}$;

Case (v) $s=(\sigma, \tau) \in S_{5}$;

Case (vi) $s=\sigma \in S_{6}$;

Case (vii) $s=\tau \in S_{7}$.

On the concrete method of determining Gevrey orders, for example, see [2] (see also Lemmas 5.1, 6.1 and Remarks 5.2, 6.2).

Remark 2.1. We can easily see that the following $s_{0}$ always satisfies the condition in Theorem 2.1 for each case:

Case (i) $s_{0}=\left(\rho_{0}, \sigma_{0}, \tau_{0}\right)$,

Case (ii) $s_{0}=\left(\rho_{0}, \sigma_{0}\right)$,

Case (iii) $s_{0}=\left(\rho_{0}, \tau_{0}\right)$,

Case (iv) $s_{0}=1^{(d)}$,

Case (v) $s_{0}=\left(\sigma_{0}, \tau_{0}\right)$,

Case (vi) $s_{0}=\sigma_{0}$,

Case (vii) $s_{0}=\tau_{0}$,

where $\rho_{0}=(\overbrace{N+1, \ldots, N+1}^{m}), \sigma_{0}=\left(\sigma_{0}^{1}, \ldots, \sigma_{0}^{k}\right), \sigma_{0}^{h}=(N+1, N+$ $\left.2, \ldots, N+n_{h}\right)(h=1, \ldots, k), \tau_{0}=(\overbrace{N+1, \ldots, N+1}^{p})$. Therefore by a linear transform of independent variables again we obtain Theorem 1.2 from Theorem 2.1 and the next Lemma 2.1. Thus the proof of Theorem 1.2 is reduced to that of Theorem 2.1.

Lemma $2.1([2]) . \quad$ Let $u(x)=\sum_{\alpha \in \mathbf{N}^{d}} u_{\alpha} x^{\alpha} \in G^{\{s, s, \ldots, s\}}(s \geq 1)$. Then for any linear transform $L: \mathbf{C}^{d} \rightarrow \mathbf{C}^{d}$, it holds that $v(y):=u(L y) \in G^{\{s, s, \ldots, s\}}$. 
From Theorem 2.1, on the existence of holomorphic solutions we obtain the following corollary.

Corollary 2.1. Let us consider Case (iii), and let us assume the following condition:

(I) $P^{\prime \prime}=P^{\prime \prime \prime}=0$

or

(II) $P^{\prime \prime \prime}=P^{\prime \prime \prime \prime}=0$.

Then under the condition (Po) the equation (2.4) has a unique holomorphic solution at the origin.

Let us consider Case (iv) and let us assume the condition (Po). Then the equation (2.5) has a unique holomorphic solution at the origin.

Remark 2.2. In Corollay 2.1, the condition (II) corresponds to the simple ideal condition in Ōshima [9].

From $\S 4$ we shall start the proof of Theorem 2.1. In $\S 4$ we shall deal with Case (iv), and in this case the proof is very simple. In $\S 5$ we shall deal with Cases (v), (vi) and (vii). However, these three cases are proved by an essentially same method, so the proof will be done only in Case (v). Finally, in $\S 6$ we prove the theorem for Cases (i), (ii) and (iii). In these cases also, the theorem is proved by an essentially same method, so we will deal with only Case (i). We remark that methods of proof employed in $\S 5$ and $\S 6$ are different.

\section{§3. Banach Spaces $G_{0, k}^{\{s\}}(R)$ and $\widetilde{G}_{0, k}^{\left\{s^{1}, s^{2}\right\}}\left(R^{1}, R^{2}\right)$}

Theorem 2.1 is proved by a contraction mapping principle in Banach spaces which consist of formal power series. For this purpose in this section we shall define two types of Banach spaces necessary in the proof, and we shall prove some fundamental facts needed later.

Definition 3.1. (1) Let $s=\left(s_{1}, \ldots, s_{d}\right) \in \mathbf{R}_{+}{ }^{d}\left(\mathbf{R}_{+}=\{r \in \mathbf{R} ; r \geq\right.$ $0\})$ and $R=\left(R_{1}, \ldots, R_{d}\right) \in\left(\mathbf{R}_{+} \backslash\{0\}\right)^{d}$, and let $k$ be a real number which satisfies $k \leq \min \left\{s_{1}, \ldots, s_{d}\right\}$. The space of formal power series $G_{0, k}^{\{s\}}(R)$ is defined as follows:

We say that $u(x)=\sum_{\alpha \in \mathbf{N}^{d}} u_{\alpha} x^{\alpha}$ belongs to $G_{0, k}^{\{s\}}(R)$ if $u(0)=0$ and

$$
\|u\|_{k, R}^{\{s\}} \equiv \sum_{|\alpha| \geq 1}\left|u_{\alpha}\right| \frac{|\alpha| !}{(s \cdot \alpha-k) !} R^{\alpha}<+\infty,
$$

where $l !=\Gamma(l+1)$ for $l \geq 0$. We remark that $k \leq \min \left\{s_{1}, \ldots, s_{d}\right\}$ implies $s \cdot \alpha-k \geq 0$ for all $\alpha$ such that $|\alpha| \geq 1$. 
(2) Let $\left(s^{1}, s^{2}\right)=\left(s^{1}{ }_{1}, \ldots, s^{1}{ }_{d_{1}}, s^{2}{ }_{1}, \ldots, s^{2}{ }_{d_{2}}\right) \in \mathbf{R}_{+}{ }^{d_{1}+d_{2}}$ and $\left(R^{1}, R^{2}\right) \in$ $\left(\mathbf{R}_{+} \backslash\{0\}\right)^{d_{1}+d_{2}}\left(R^{1}=\left(R_{1}^{1}, \ldots, R_{d_{1}}^{1}\right), R^{2}=\left(R^{2}{ }_{1}, \ldots, R_{d_{2}}^{2}\right)\right)$, and let $k$ be a real number which satisfies $k \leq \min \left\{s^{1}{ }_{1}, \ldots, s^{1}{ }_{d_{1}}, s^{2}{ }_{1}, \ldots, s^{2}{ }_{d_{2}}\right\}$. The space of formal power series $\widetilde{G}_{0, k}^{\left\{s^{1}, s^{2}\right\}}\left(R^{1}, R^{2}\right)$ is defined as follows:

We say that $u(x, y)=\sum_{(\alpha, \beta) \in \mathbf{N}^{d_{1}+d_{2}}} u_{\alpha \beta} x^{\alpha} y^{\beta}$ belongs to $\widetilde{G}_{0, k}^{\left\{s^{1}, s^{2}\right\}}\left(R^{1}, R^{2}\right)$ if $u(0,0)=0$ and

$$
\left\||| u\left|\|_{k, R^{1}, R^{2}}^{\left\{s^{1}, s^{2}\right\}} \equiv \sum_{|\alpha|+|\beta| \geq 1}\right| u_{\alpha \beta} \mid \frac{|\alpha| !|\beta| !}{\left(s^{1} \cdot \alpha+s^{2} \cdot \beta-k\right) !}\left(R^{1}\right)^{\alpha}\left(R^{2}\right)^{\beta}<+\infty .\right.
$$

Then $G_{0, k}^{\{s\}}(R)$ and $\widetilde{G}_{0, k}^{\left\{s^{1}, s^{2}\right\}}\left(R^{1}, R^{2}\right)$ are Banach spaces equipped with the norms $\|\cdot\|_{k, R}^{\{s\}}$ and $\|\mid \cdot\| \|_{k, R^{1}, R^{2}}^{\left\{s^{1}, s^{2}\right\}}$, respectively.

Remark 3.1. In [3], we introduced the spaces $G_{0,0}^{\{s\}}(R)$ and $\widetilde{G}_{0,0}^{\left\{s^{1}, s^{2}\right\}}\left(R^{1}\right.$, $R^{2}$ ). In the proof of the theorem for quasi-linear equations (in particular, when we prove divergence of the formal solution), the spaces $G_{0, k}^{\{s\}}(R)$ and $\widetilde{G}_{0, k}^{\left\{s^{1}, s^{2}\right\}}\left(R^{1}, R^{2}\right)$ for a positive $k$ play significant roles (cf. $\S 5$ and $\left.\S 6\right)$.

Lemma 3.1. (1) Let $s \in \mathbf{R}_{+}{ }^{d}$ and let $k \leq \min \left\{s_{1}, \ldots, s_{d}\right\}$. Then for all $R \in\left(\mathbf{R}_{+} \backslash\{0\}\right)^{d}$ it holds that

$$
G_{0, k}^{\{s\}}(R) \subset G^{\{s\}} .
$$

(2) Let $\left(s^{1}, s^{2}\right) \in \mathbf{R}_{+}{ }^{d_{1}+d_{2}}$ and let $k \leq \min \left\{s^{1}{ }_{1}, \ldots, s^{1}{ }_{d_{1}}, s^{2}{ }_{1}, \ldots, s^{2}{ }_{d_{2}}\right\}$. Then for all $\left(R^{1}, R^{2}\right) \in\left(\mathbf{R}_{+} \backslash\{0\}\right)^{d_{1}+d_{2}}$ it holds that

$$
\widetilde{G}_{0, k}^{\left\{s^{1}, s^{2}\right\}}\left(R^{1}, R^{2}\right) \subset G^{\left\{s^{1}, s^{2}\right\}} .
$$

Proof. (1): In general it follows from Cauchy's integral formula that a formal power series $u(x)=\sum_{\alpha \in \mathbf{N}^{d}} u_{\alpha} x^{\alpha}$ belongs to $G^{\{s\}}$ if and only if there exist some positive constants $A$ and $B$ such that

$$
\left|u_{\alpha}\right| \leq A B^{|\alpha|}(\alpha !)^{s-1^{(d)}} \text { for all } \alpha \in \mathbf{N}^{d} .
$$

Now let $u(x)=\sum_{|\alpha| \geq 1} u_{\alpha} x^{\alpha} \in G_{0, k}^{\{s\}}(R)$. Then we have

$$
\left|u_{\alpha}\right| \leq\|u\|_{k, R}^{\{s\}} \frac{(s \cdot \alpha-k) !}{|\alpha| !} \cdot \frac{1}{R^{\alpha}} \leq\|u\|_{k, R}^{\{s\}} \frac{(s \cdot \alpha-k) !}{(\alpha !)^{s}} \cdot \frac{1}{R^{\alpha}} \cdot(\alpha !)^{s-1^{(d)}} .
$$

By Stirling's formula there exist some positive constants $A$ and $B$ such that

$$
\frac{(s \cdot \alpha-k) !}{(\alpha !)^{s}} \leq A \cdot B^{|\alpha|} \text { for all } \alpha(|\alpha| \geq 1) .
$$


This completes the proof.

(2) is also proved similarly to (1).

Lemma 3.2. Let $a(x)=\sum_{\alpha \in \mathbf{N}^{d}} a_{\alpha} x^{\alpha}$ and $a(x, y)=$ $\sum_{(\alpha, \beta) \in \mathbf{N}^{d_{1}+d_{2}}} a_{\alpha \beta} x^{\alpha} y^{\beta}$ be holomorphic at $x=0$ and $(x, y)=(0,0)$, respectively.

(1) Let $s \in[1,+\infty)^{d}$ and $k \leq s_{i}-1$ for all $i=1, \ldots, d$. If $a(R)$ converges, then for all $u(x) \in G_{0, k}^{\{s\}}(R)$ it holds that $a(x) \cdot u(x) \in G_{0, k}^{\{s\}}(R)$ and

$$
\|a \cdot u\|_{k, R}^{\{s\}} \leq|a|(R) \cdot\|u\|_{k, R}^{\{s\}},
$$

where $|a|(R):=\sum_{\alpha \in \mathbf{N}^{d}}\left|a_{\alpha}\right| R^{\alpha}$.

$(1)^{\prime}$ Let $s$ and $k$ be same as in $(1)$, and let us assume that $a(R)$ converges. If $a(0)=0$, then it holds that $a(x) \in G_{0, k}^{\{s\}}(R)$ and

$$
\|a\|_{k, R}^{\{s\}} \leq|a|(R) .
$$

(2) Let $\left(s^{1}, s^{2}\right) \in[1,+\infty)^{d_{1}+d_{2}}$ and $k \leq s^{1}{ }_{i}-1, s^{2}{ }_{j}-1$ for all $i=$ $1, \ldots, d_{1}$ and $j=1, \ldots, d_{2}$. If $a\left(R^{1}, R^{2}\right)$ converges, then for all $u(x, y) \in$ $\widetilde{G}_{0, k}^{\left\{s^{1}, s^{2}\right\}}\left(R^{1}, R^{2}\right)$ it holds that $a(x, y) \cdot u(x, y) \in \widetilde{G}_{0, k}^{\left\{s^{1}, s^{2}\right\}}\left(R^{1}, R^{2}\right)$ and

$$
\left\|| a \cdot u \| \| _ { k , R ^ { 1 } , R ^ { 2 } } ^ { \{ s ^ { 1 } , s ^ { 2 } \} } \leq | a \left|\left(R^{1}, R^{2}\right) \cdot\||u|\|_{k, R^{1}, R^{2}}^{\left\{s^{1}, s^{2}\right\}},\right.\right.
$$

where $|a|\left(R^{1}, R^{2}\right):=\sum_{(\alpha, \beta) \in \mathbf{N}^{d_{1}+d_{2}}}\left|a_{\alpha \beta}\right|\left(R^{1}\right)^{\alpha}\left(R^{2}\right)^{\beta}$.

$(2)^{\prime}$ Let $\left(s^{1}, s^{2}\right)$ and $k$ be same as in $(2)$, and let us assume that $a\left(R^{1}, R^{2}\right)$ converges. If $a(0,0)=0$, then it holds that $a(x, y) \in \widetilde{G}_{0, k}^{\left\{s^{1}, s^{2}\right\}}\left(R^{1}, R^{2}\right)$ and

$$
\||a|\|_{k, R^{1}, R^{2}}^{\left\{s^{1}, s^{2}\right\}} \leq|a|\left(R^{1}, R^{2}\right) .
$$

Proof. (1): Since

$$
a(x) \cdot u(x)=\sum_{\alpha, \beta \in \mathbf{N}^{d},|\beta| \geq 1} a_{\alpha} u_{\beta} x^{\alpha+\beta},
$$

it holds that

$$
\|a \cdot u\|_{k, R}^{\{s\}}=\sum_{\alpha, \beta \in \mathbf{N}^{d},|\beta| \geq 1}\left|a_{\alpha}\right| \cdot\left|u_{\beta}\right| \frac{|\alpha+\beta| !}{\{s \cdot(\alpha+\beta)-k\} !} R^{\alpha+\beta} .
$$

Hence it is sufficient to prove that

$$
\frac{|\alpha+\beta| !}{|\beta| !} \leq \frac{(s \cdot \alpha+s \cdot \beta-k) !}{(s \cdot \beta-k) !}, \text { for all } \alpha, \beta \in \mathbf{N}^{d}(|\beta| \geq 1) \text {. }
$$

This is proved as follows: $s \in[1,+\infty)^{d}$ and $k \leq s_{i}-1(i=1, \ldots, d)$ imply that 
$s \cdot \beta-k \geq|\beta|$ for all $\beta$ such that $|\beta| \geq 1$. Moreover it is clear that $|\alpha| \leq s \cdot \alpha$ for all $\alpha \in \mathbf{N}^{d}$. Therefore we obtain

$$
\begin{aligned}
\frac{|\alpha+\beta| !}{|\beta| !} & =\prod_{j=1}^{|\alpha|}(j+|\beta|) \leq \prod_{j=1}^{|\alpha|}(j+s \cdot \beta-k) \\
& =\frac{(|\alpha|+s \cdot \beta-k) !}{(s \cdot \beta-k) !} \leq \frac{(s \cdot \alpha+s \cdot \beta-k) !}{(s \cdot \beta-k) !} .
\end{aligned}
$$

(2) is also proved by noting that

$$
\begin{aligned}
\frac{\left|\alpha^{1}+\alpha^{2}\right| !}{\left|\alpha^{2}\right| !} \frac{\left|\beta^{1}+\beta^{2}\right| !}{\left|\beta^{2}\right| !} & \leq \frac{\left(\left|\alpha^{1}+\alpha^{2}\right|+\left|\beta^{1}+\beta^{2}\right|\right) !}{\left(\left|\alpha^{2}\right|+\left|\beta^{2}\right|\right) !}=\prod_{j=1}^{\left|\alpha^{1}\right|+\left|\beta^{1}\right|}\left(j+\left|\alpha^{2}\right|+\left|\beta^{2}\right|\right) \\
& \leq \prod_{j=1}^{\left|\alpha^{1}\right|+\left|\beta^{1}\right|}\left(j+s^{1} \cdot \alpha^{2}+s^{2} \cdot \beta^{2}-k\right) \\
& =\frac{\left(\left|\alpha^{1}\right|+\left|\beta^{1}\right|+s^{1} \cdot \alpha^{2}+s^{2} \cdot \beta^{2}-k\right) !}{\left(s^{1} \cdot \alpha^{2}+s^{2} \cdot \beta^{2}-k\right) !} \\
& \leq \frac{\left\{s^{1} \cdot\left(\alpha^{1}+\alpha^{2}\right)+s^{2} \cdot\left(\beta^{1}+\beta^{2}\right)-k\right\} !}{\left(s^{1} \cdot \alpha^{2}+s^{2} \cdot \beta^{2}-k\right) !}
\end{aligned}
$$

for all $\left(\alpha^{1}, \beta^{1}\right),\left(\alpha^{2}, \beta^{2}\right) \in \mathbf{N}^{d_{1}+d_{2}}\left(\left|\alpha^{2}\right|+\left|\beta^{2}\right| \geq 1\right)$.

$(1)^{\prime}$ is clear from the inequality $s \cdot \alpha-k \geq|\alpha|$ for all $\alpha$ such that $|\alpha| \geq 1$. $(2)^{\prime}$ is also obvious.

Lemma 3.3. (1) Let $s \in[1,+\infty)^{d}, 0 \leq k \leq s_{i}-1(i=1, \ldots, d)$ and let us assume that $u(x), v(x) \in G_{0, k}^{\{s\}}(R)$. Then it holds that $u(x) \cdot v(x) \in G_{0, k}^{\{s\}}(R)$ and

$$
\|u \cdot v\|_{k, R}^{\{s\}} \leq \mathrm{S}\|u\|_{k, R}^{\{s\}} \cdot\|v\|_{k, R}^{\{s\}}
$$

where $\mathbf{S}=\max \left\{s_{1}, \ldots, s_{d}\right\}$.

(2) Let $\left(s^{1}, s^{2}\right) \in[1,+\infty)^{d_{1}+d_{2}}, 0 \leq k \leq s^{1}{ }_{i}-1, s^{2}{ }_{j}-1\left(i=1, \ldots, d_{1}\right.$; $\left.j=1, \ldots, d_{2}\right)$ and let us assume that $u(x, y), v(x, y) \in \widetilde{G}_{0, k}^{\left\{s^{1}, s^{2}\right\}}\left(R^{1}, R^{2}\right)$. Then it holds that $u(x, y) \cdot v(x, y) \in \widetilde{G}_{0, k}^{\left\{s^{1}, s^{2}\right\}}\left(R^{1}, R^{2}\right)$ and

$$
\left.\|u \cdot v\|\right|_{k, R^{1}, R^{2}} ^{\left\{s^{1}, s^{2}\right\}} \leq \widetilde{\mathrm{S}}\|u\|\left\|_{k, R^{1}, R^{2}}^{\left\{s^{1}, s^{2}\right\}} \cdot\right\| v \|_{k, R^{1}, R^{2}}^{\left\{s^{1}, s^{2}\right\}}
$$

where $\widetilde{\mathrm{S}}=\max \left\{s^{1}{ }_{1}, \ldots, s^{1}{ }_{d_{1}}, s^{2}{ }_{1}, \ldots, s^{2}{ }_{d_{2}}\right\}$.

Proof. First of all, we remark that in general the Beta function

$$
B(m, n)=\int_{0}^{1} t^{m-1}(1-t)^{n-1} d t
$$


has the following property:

$$
0<m_{1}<m_{2}, 0<n_{1}<n_{2} \Rightarrow B\left(m_{1}, n_{1}\right)>B\left(m_{2}, n_{2}\right) .
$$

Moreover we remark that the following equality holds: For $m, n>0$,

$$
\frac{m ! n !}{(m+n) !}=B(m+1, n+1) \cdot(m+n+1) .
$$
have

(1): Let $u(x)=\sum_{|\alpha| \geq 1} u_{\alpha} x^{\alpha}, v(x)=\sum_{|\beta| \geq 1} v_{\beta} x^{\beta} \in G_{0, k}^{\{s\}}(R)$. Then we

$$
\|u \cdot v\|_{k, R}^{\{s\}}=\sum_{|\alpha|,|\beta| \geq 1}\left|u_{\alpha}\right| \cdot\left|v_{\beta}\right| \frac{|\alpha+\beta| !}{\{s \cdot(\alpha+\beta)-k\} !} R^{\alpha+\beta} .
$$

Here it follows from the above remark that

$$
\begin{aligned}
\frac{(s \cdot \alpha-k) !(s \cdot \beta-k) !}{\{s \cdot(\alpha+\beta)-k\} !} \leq & \frac{(s \cdot \alpha-k) !(s \cdot \beta-k) !}{\{s \cdot(\alpha+\beta)-2 k\} !} \quad(\text { since } k \geq 0) \\
= & B(s \cdot \alpha-k+1, s \cdot \beta-k+1) \cdot\{s \cdot(\alpha+\beta)-2 k+1\} \\
\leq & B(|\alpha|+1,|\beta|+1) \cdot\{s \cdot(\alpha+\beta)-2 k+1\} \\
& \left(\text { since } k \leq\left(s-1^{(d)}\right) \cdot \alpha,\left(s-1^{(d)}\right) \cdot \beta \text { for }|\alpha|,|\beta| \geq 1\right) \\
= & \frac{|\alpha| !|\beta| !}{|\alpha+\beta| !} \cdot \frac{s \cdot(\alpha+\beta)-2 k+1}{|\alpha|+|\beta|+1} \\
\leq & \mathrm{S} \cdot \frac{|\alpha| !|\beta| !}{|\alpha+\beta| !} .
\end{aligned}
$$

Therefore we have obtained (3.1).

(2): Let $u(x, y)=\sum_{|\alpha|+|\beta| \geq 1} u_{\alpha \beta} x^{\alpha} y^{\beta}, v(x, y)=\sum_{|\gamma|+|\delta| \geq 1} v_{\gamma \delta} x^{\gamma} y^{\delta} \in$ $\widetilde{G}_{0, k}^{\left\{s^{1}, s^{2}\right\}}\left(R^{1}, R^{2}\right)$. Then we have

$\|u \cdot v\| \|_{k, R^{1}, R^{2}}^{\left\{s^{1}, s^{2}\right\}}$

$$
=\sum_{\substack{|\alpha|+|\beta| \geq 1 \\|\gamma|+|\delta| \geq 1}}\left|u_{\alpha \beta}\right| \cdot\left|v_{\gamma \delta}\right| \frac{|\alpha+\gamma| !|\beta+\delta| !}{\left\{s^{1} \cdot(\alpha+\gamma)+s^{2} \cdot(\beta+\delta)-k\right\} !}\left(R^{1}\right)^{\alpha+\gamma}\left(R^{2}\right)^{\beta+\delta} .
$$

Here similarly to (1), it holds that

$$
\frac{\left(s^{1} \cdot \alpha+s^{2} \cdot \beta-k\right) !\left(s^{1} \cdot \gamma+s^{2} \cdot \delta-k\right) !}{\left\{s^{1} \cdot(\alpha+\gamma)+s^{2} \cdot(\beta+\delta)-k\right\} !} \leq \widetilde{\mathrm{S}} \cdot \frac{(|\alpha|+|\beta|) !(|\gamma|+|\delta|) !}{(|\alpha+\gamma|+|\beta+\delta|) !} .
$$

Moreover it holds that

$$
\frac{(|\alpha|+|\beta|) !(|\gamma|+|\delta|) !}{(|\alpha+\gamma|+|\beta+\delta|) !} \leq \frac{|\alpha| !|\beta| !}{|\alpha+\gamma| !} \cdot \frac{|\gamma| !|\delta| !}{|\beta+\delta| !}
$$


(see the proof of Lemma 3.3, (2) in [3]), which implies that

$$
\begin{aligned}
& \frac{|\alpha+\gamma| !|\beta+\delta| !}{\left\{s^{1} \cdot(\alpha+\gamma)+s^{2} \cdot(\beta+\delta)-k\right\} !} \\
& \leq \widetilde{\mathrm{S}} \cdot \frac{|\alpha| !|\beta| !}{\left\{s^{1} \cdot \alpha+s^{2} \cdot \beta-k\right\} !} \cdot \frac{|\gamma| !|\delta| !}{\left\{s^{1} \cdot \gamma+s^{2} \cdot \delta-k\right\} !} .
\end{aligned}
$$

Therefore (3.2) is proved.

Lemma 3.4. (1) Let $s \in[1,+\infty)^{d}$ and $0 \leq k \leq s_{i}-1(i=1, \ldots, d)$, and let $\mathrm{S}$ be same as in Lemma 3.3, (1). Let $F(x, u)$ be a holomorphic function at $(x, u)=(0,0)$, which satisfies $F(0,0)=0$. Let

$$
F(x, u)=\sum_{|\alpha|+r \geq 1} F_{\alpha r} x^{\alpha} u^{r}
$$

be the Taylor expansion of $F(x, u)$. Moreover let us define the power series $|F|(x, u)$ by

$$
|F|(x, u)=\sum_{|\alpha|+r \geq 1}\left|F_{\alpha r}\right| x^{\alpha} u^{r}
$$

If $u(x) \in G_{0, k}^{\{s\}}(R)$, then it holds that $F(x, u(x)) \in G_{0, k}^{\{s\}}(R)$ and

$$
\|F(x, u(x))\|_{k, R}^{\{s\}} \leq \frac{1}{\mathrm{~S}}|F|\left(R, \mathrm{~S}\|u\|_{k, R}^{\{s\}}\right)
$$

as far as both sides are well-defined.

(2) Let $\left(s^{1}, s^{2}\right) \in[1,+\infty)^{d_{1}+d_{2}}$ and $0 \leq k \leq s^{1}{ }_{i}-1, s^{2}{ }_{j}-1\left(i=1, \ldots, d_{1}\right.$; $\left.j=1, \ldots, d_{2}\right)$, and let $\widetilde{\mathrm{S}}$ be same as in Lemma 3.3, (2). Let $F(x, y, u)$ be a holomorphic function at $(x, y, u)=(0,0,0)$, which satisfies $F(0,0,0)=0$. Let $F(x, y, u)=\sum_{|\alpha|+|\beta|+r \geq 1} F_{\alpha \beta r} x^{\alpha} y^{\beta} u^{r}$ be the Taylor expansion of $F(x, y, u)$. Moreover let us define the power series $|F|(x, y, u)$ by $|F|(x, y, u)=$ $\sum_{|\alpha|+|\beta|+r \geq 1}\left|F_{\alpha \beta r}\right| x^{\alpha} y^{\beta} u^{r}$.

If $u(x, y) \in \widetilde{G}_{0, k}^{\left\{s^{1}, s^{2}\right\}}\left(R^{1}, R^{2}\right)$, then it holds that $F(x, y, u(x, y)) \in$ $\widetilde{G}_{0, k}^{\left\{s^{1}, s^{2}\right\}}\left(R^{1}, R^{2}\right)$ and

$$
\left\|\left|F(x, y, u(x, y)) \|_{k, R^{1}, R^{2}}^{\left\{s^{1}, s^{2}\right\}} \leq \frac{1}{\widetilde{\mathrm{S}}}\right| F \mid\left(R^{1}, R^{2}, \widetilde{\mathrm{S}}\||u|\|_{k, R^{1}, R^{2}}^{\left\{s^{1}, s^{2}\right\}}\right)\right.
$$

as far as both sides are well-defined.

Proof. (1): It follows from Lemma 3.2, (1), (1)' and Lemma 3.3, (1) that

$$
\|F(x, u(x))\|_{k, R}^{\{s\}} \leq \sum_{|\alpha|+r \geq 1}\left|F_{\alpha r}\right| R^{\alpha}\left\|u^{r}\right\|_{k, R}^{\{s\}}
$$




$$
\begin{aligned}
& \leq \frac{1}{\mathrm{~S}} \sum_{|\alpha|+r \geq 1}\left|F_{\alpha r}\right| R^{\alpha}\left(\mathrm{S}\|u\|_{k, R}^{\{s\}}\right)^{r} \\
& =\frac{1}{\mathrm{~S}}|F|\left(R, \mathrm{~S}\|u\|_{k, R}^{\{s\}}\right) .
\end{aligned}
$$

(2) is also proved similarly to (1) by using Lemma 3.2, (2), (2)' and Lemma $3.3,(2)$.

Similarly to the proof of Lemma 3.4 we have the following.

Lemma 3.5. (1) Let $s, k$ and $\mathrm{S}$ be same as in Lemma 3.4, (1). Let $\mathcal{F}(x, u)$ be holomorphic at $(x, u)=(0,0)$.

If $u(x), v(x) \in G_{0, k}^{\{s\}}(R)$, then it holds that $\mathcal{F}(x, u(x)) v(x) \in G_{0, k}^{\{s\}}(R)$ and

$$
\|\mathcal{F}(x, u(x)) v(x)\|_{k, R}^{\{s\}} \leq|\mathcal{F}|\left(R, \mathrm{~S}\|u\|_{k, R}^{\{s\}}\right) \cdot\|v\|_{k, R}^{\{s\}} .
$$

(2) Let $\left(s^{1}, s^{2}\right), k$ and $\widetilde{\mathrm{S}}$ be same as in Lemma $3.4,(2)$. Let $\mathcal{F}(x, y, u)$ be holomorphic at $(x, y, u)=(0,0,0)$.

If $u(x, y), v(x, y) \in \widetilde{G}_{0, k}^{\left\{s^{1}, s^{2}\right\}}\left(R^{1}, R^{2}\right)$, then $\mathcal{F}(x, y, u(x, y)) v(x, y) \in$ $\widetilde{G}_{0, k}^{\left\{s^{1}, s^{2}\right\}}\left(R^{1}, R^{2}\right)$ and

$$
\| \mathcal{F}(x, y, u(x, y)) v(x, y)||_{k, R^{1}, R^{2}}^{\left\{s^{1}, s^{2}\right\}} \leq|\mathcal{F}|\left(R^{1}, R^{2}, \widetilde{S}|\|u\||_{k, R^{1}, R^{2}}^{\left\{s^{1}, s^{2}\right\}} \cdot\|\| v\|\|_{k, R^{1}, R^{2}}^{\left\{s^{1}, s^{2}\right\}}\right.
$$

Lemma 3.6. (1) Let $s, k, \mathrm{~S}$ and $F(x, u)$ be same as in Lemma 3.4, (1). Moreover let us assume that $F(x, u)$ has the following Taylor expansion:

$$
F(x, u)=\sum_{|\alpha|+r \geq 2} F_{\alpha r} x^{\alpha} u^{r} .
$$

If $u(x), v(x) \in G_{0, k}^{\{s\}}(R)$, then we have

$$
\|F(x, u(x))-F(x, v(x))\|_{k, R}^{\{s\}} \leq\|u-v\|_{k, R}^{\{s\}} \times\left|F_{u}\right|\left(R, \mathrm{~S}\left(\|u\|_{k, R}^{\{s\}}+\|v\|_{k, R}^{\{s\}}\right)\right),
$$

where $F_{u}(x, u)=(\partial / \partial u) F(x, u)=\sum_{|\alpha|+r \geq 2, r \geq 1} r F_{\alpha r} x^{\alpha} u^{r-1}$ and $\left|F_{u}\right|(x, u)$ is the power series defined by $\left|F_{u}\right|(x, u)=\sum_{|\alpha|+r \geq 2, r \geq 1} r\left|F_{\alpha r}\right| x^{\alpha} u^{r-1}$.

(2) Let $\left(s^{1}, s^{2}\right), k, \widetilde{\mathrm{S}}$ and $F(x, y, u)$ be same as in Lemma 3.4, (2). Moreover let us assume that $F(x, y, u)$ has the following Taylor expansion:

$$
F(x, y, u)=\sum_{|\alpha|+|\beta|+r \geq 2} F_{\alpha \beta r} x^{\alpha} y^{\beta} u^{r} .
$$


If $u(x, y), v(x, y) \in \widetilde{G}_{0, k}^{\left\{s^{1}, s^{2}\right\}}\left(R^{1}, R^{2}\right)$, then we have

$$
\begin{aligned}
& \|\| F(x, y, u(x, y))-F(x, y, v(x, y)) \mid \|_{k, R^{1}, R^{2}}^{\left\{s^{1}, s^{2}\right\}} \\
& \quad \leq\left\||| u-v\left|\|_{k, R^{1}, R^{2}}^{\left\{s^{1}, s^{2}\right\}} \times\right| F_{u} \mid\left(R^{1}, R^{2}, \widetilde{\mathrm{S}}\left(\left\||| u\left|\left\|_{k, R^{1}, R^{2}}^{\left\{s^{1}, s^{2}\right\}}+\right\|\right|\right\| \|_{k, R^{1}, R^{2}}^{\left\{s^{1}, s^{2}\right\}}\right),\right.\right.
\end{aligned}
$$

where $F_{u}(x, y, u)=(\partial / \partial u) F(x, y, u)=\sum_{|\alpha|+|\beta|+r \geq 2, r \geq 1} r F_{\alpha \beta r} x^{\alpha} y^{\beta} u^{r-1}$ and $\left|F_{u}\right|(x, y, u)$ is the power series defined by $\left|F_{u}\right|(x, y, u)=$ $\sum_{|\alpha|+|\beta|+r \geq 2, r \geq 1} r\left|F_{\alpha \beta r}\right| x^{\alpha} y^{\beta} u^{r-1}$.

Proof. (1): Since

$$
F(x, u)-F(x, v)=(u-v) \int_{0}^{1} F_{u}(x, \theta u+(1-\theta) v) d \theta,
$$

it follows from Lemma 3.3, (1) and Lemma 3.4, (1) that

$$
\begin{aligned}
\|F(x, u(x))-F(x, v(x))\|_{k, R}^{\{s\}} & \leq \mathrm{S}\|u-v\|_{k, R}^{\{s\}} \times\left\|\int_{0}^{1} F_{u}(x, \theta u(x)+(1-\theta) v(x)) d \theta\right\|_{k, R}^{\{s\}} \\
& \leq\|u-v\|_{k, R}^{\{s\}} \times \int_{0}^{1}\left|F_{u}\right|\left(R, \mathrm{~S}\|\theta u+(1-\theta) v\|_{k, R}^{\{s\}}\right) d \theta \\
& \leq\|u-v\|_{k, R}^{\{s\}} \times\left|F_{u}\right|\left(R, \mathrm{~S}\left(\|u\|_{k, R}^{\{s\}}+\|v\|_{k, R}^{\{s\}}\right)\right) .
\end{aligned}
$$

(2) is also proved similarly to (1) by using Lemma 3.3, (2) and Lemma 3.4, (2).

Lemma 3.7. (1) Let $s, k, \mathrm{~S}$ and $\mathcal{F}(x, u)$ be same as in Lemma 3.5, (1).

If $u_{1}(x), u_{2}(x), v_{1}(x), v_{2}(x) \in G_{0, k}^{\{s\}}(R)$, then it holds that

$$
\begin{aligned}
&\left\|\mathcal{F}\left(x, u_{1}(x)\right) u_{2}(x)-\mathcal{F}\left(x, v_{1}(x)\right) v_{2}(x)\right\|_{k, R}^{\{s\}} \\
& \leq \mathrm{S}\left\|u_{1}-v_{1}\right\|_{k, R}^{\{s\}} \times\left|\mathcal{F}_{u}\right|\left(R, \mathrm{~S}\left(\left\|u_{1}\right\|_{k, R}^{\{s\}}+\left\|v_{1}\right\|_{k, R}^{\{s\}}\right)\right) \cdot\left\|u_{2}\right\|_{k, R}^{\{s\}} \\
& \quad+\left\|u_{2}-v_{2}\right\|_{k, R}^{\{s\}} \times|\mathcal{F}|\left(R, \mathrm{~S}\left\|v_{1}\right\|_{k, R}^{\{s\}}\right) .
\end{aligned}
$$

(2) Let $\left(s^{1}, s^{2}\right), k, \widetilde{\mathrm{S}}$ and $\mathcal{F}(x, y, u)$ be same as in Lemma 3.5, (2).

If $u_{1}(x, y), u_{2}(x, y), v_{1}(x, y), v_{2}(x, y) \in \widetilde{G}_{0, k}^{\left\{s^{1}, s^{2}\right\}}\left(R^{1}, R^{2}\right)$, then it holds that

$$
\left\|\mid \mathcal{F}\left(x, y, u_{1}(x, y)\right) u_{2}(x, y)-\mathcal{F}\left(x, y, v_{1}(x, y)\right) v_{2}(x, y)\right\| \|_{k, R^{1}, R^{2}}^{\left\{s^{1}, s^{2}\right\}}
$$




$$
\begin{gathered}
\leq\left.\widetilde{\mathrm{S}}||\left|u_{1}-v_{1}\right|\right|_{k, R^{1}, R^{2}} ^{\left\{s^{1}, s^{2}\right\}} \times\left|\mathcal{F}_{u}\right|\left(R^{1}, R^{2}, \widetilde{\mathrm{S}}\left(||\left|u_{1}\right|\left\|_{k, R^{1}, R^{2}}^{\left\{s^{1}, s^{2}\right\}}+\right\|\left|v_{1}\right| \|_{k, R^{1}, R^{2}}^{\left\{s^{1}, s^{2}\right\}}\right)\right) \\
\times\left\|\left.|| u_{2}||\right|_{k, R^{1}, R^{2}} ^{\left\{s^{1}, s^{2}\right\}}+\right\||| u_{2}-v_{2}||_{k, R^{1}, R^{2}}^{\left\{s^{1}, s^{2}\right\}} \times|\mathcal{F}|\left(R^{1}, R^{2}, \widetilde{\mathrm{S}}||\left|v_{1}\right|||_{k, R^{1}, R^{2}}^{\left\{s^{1}, s^{2}\right\}}\right) .
\end{gathered}
$$

Proof. (1): It is proved similarly to the previous lemmas by using the following equality:

$$
\begin{aligned}
\mathcal{F} & \left(x, u_{1}\right) u_{2}-\mathcal{F}\left(x, v_{1}\right) v_{2} \\
& =\left\{\mathcal{F}\left(x, u_{1}\right)-\mathcal{F}\left(x, v_{1}\right)\right\} u_{2}+\left(u_{2}-v_{2}\right) \mathcal{F}\left(x, v_{1}\right) \\
& =\left(u_{1}-v_{1}\right) \int_{0}^{1} \mathcal{F}_{u}\left(x, \theta u_{1}+(1-\theta) v_{1}\right) d \theta \cdot u_{2}+\left(u_{2}-v_{2}\right) \mathcal{F}\left(x, v_{1}\right) .
\end{aligned}
$$

(2): Similar to (1).

\section{$\S 4$. Proof of Theorem 2.1 (Case (iv))}

Let us start the proof of Theorem 2.1. As mentioned in $\S 1$, we omit the proof of the unique existence of the formal solution. From $\S 4$ to $\S 6$, admitting the unique existence of the formal solution, we will prove its Gevrey order. In this section we consider Case (iv), and we shall prove that the formal solution $u(x)=\sum_{|\alpha| \geq 1} u_{\alpha} x^{\alpha}$ of the equation (2.5) converges near $x=0$, that is, $u \in$ $G^{\left\{1^{(d)}\right\}}$. For the proof we use the Banach space $G_{0,0}^{\left\{1^{(d)}\right\}}(X)\left(X=\left(X_{1}, \ldots, X_{d}\right) \in\right.$ $\left.\left(\mathbf{R}_{+} \backslash\{0\}\right)^{d}\right)$. For simplicity we write this space as $\mathcal{O}_{0}(X)$ and write the norm $\|\cdot\|_{0, X}^{\left\{1^{(d)}\right\}}$ as $\|\cdot\|_{X}$. Therefore $u(x) \in \mathcal{O}_{0}(X)$ means

$$
\|u\|_{X} \equiv \sum_{|\alpha| \geq 1}\left|u_{\alpha}\right| X^{\alpha}<+\infty
$$

Proof of Case (iv) of Theorem 2.1. First we remark that the operator $\Lambda$ defined in (2.2) is well-defined as the mapping from $G^{\left\{1^{(d)}\right\}}$ to itself. Moreover since the condition (Po) implies that $\lambda \cdot \alpha-f_{u}(0,0) \neq 0$ for all $\alpha \in \mathbf{N}^{d}$, where $\lambda \cdot \alpha=\sum_{i=1}^{d} \lambda_{i} \alpha_{i}$, the operator $\Lambda: G^{\left\{1^{(d)}\right\}} \rightarrow G^{\left\{1^{(d)}\right\}}$ is bijective and $\Lambda^{-1}$ is given by

$$
\Lambda^{-1}\left(\sum_{\alpha \in \mathbf{N}^{d}} U_{\alpha} x^{\alpha}\right)=\sum_{\alpha \in \mathbf{N}^{d}} \frac{U_{\alpha}}{\lambda \cdot \alpha-f_{u}(0,0)} x^{\alpha} .
$$

Now we introduce a new unknown function $U(x)$ by

$$
U(x)=\Lambda u(x), \text { that is, } u(x)=\Lambda^{-1} U(x) .
$$


Then the equation (2.5) is equivalent to the following one:

$$
U(x)=T U(x), \quad U(0)=0,
$$

where

$$
\begin{aligned}
T U(x)= & -\sum_{i=1}^{m-1} \delta_{i} x_{i+1} \frac{\partial \Lambda^{-1} U}{\partial x_{i}}(x) \\
& +\sum_{i=1}^{m}\left(\sum_{|\alpha|+r \geq 2}^{\text {finite }} c_{i \alpha r}\left(x, \Lambda^{-1} U(x)\right) x^{\alpha}\left(\Lambda^{-1} U(x)\right)^{r}\right) \frac{\partial \Lambda^{-1} U}{\partial x_{i}}(x) \\
& +g_{0}(x)+g\left(x, \Lambda^{-1} U(x)\right) .
\end{aligned}
$$

Let us write the $\varepsilon$-closed ball in $\mathcal{O}_{0}(X)$ as

$$
\mathcal{O}_{0}(X ; \varepsilon)=\left\{U(x)=\sum_{|\alpha| \geq 1} U_{\alpha} x^{\alpha} \in \mathcal{O}_{0}(X) ;\|U\|_{X} \leq \varepsilon\right\} .
$$

We shall prove that the operator $T$ is well-defined as the mapping from $\mathcal{O}_{0}(X ; \varepsilon)$ to itself by choosing $X$ and $\varepsilon$ suitably and that it becomes a contraction mapping there.

By the condition $(\mathrm{Po})$ there exists a positive constant $C$ such that

$$
\frac{1}{\left|\lambda \cdot \alpha-f_{u}(0,0)\right|} \leq C, \quad \frac{|\alpha|}{\left|\lambda \cdot \alpha-f_{u}(0,0)\right|} \leq C \text { for all } \alpha \in \mathbf{N}^{d}
$$

By the first inequality the linear operator $\Lambda^{-1}: \mathcal{O}_{0}(X) \rightarrow \mathcal{O}_{0}(X)$ is bounded and we have

$$
\left\|\Lambda^{-1} U\right\|_{X} \leq C\|U\|_{X} \text { for } U(x) \in \mathcal{O}_{0}(X)
$$

Here let us give some lemmas.

Lemma 4.1. Let us assume that $|\alpha|+r \geq 1$, and let $U_{1}, \ldots, U_{r}$ and $V$ belong to $\mathcal{O}_{0}(X)$. Then $x^{\alpha}\left(\Lambda^{-1} U_{1}\right) \cdots\left(\Lambda^{-1} U_{r}\right) \partial_{x_{i}} \Lambda^{-1} V$ belongs to $\mathcal{O}_{0}(X)$ and it holds that

$$
\left\|x^{\alpha}\left(\Lambda^{-1} U_{1}\right) \cdots\left(\Lambda^{-1} U_{r}\right) \partial_{x_{i}} \Lambda^{-1} V\right\|_{X} \leq C^{r+1} \frac{X^{\alpha}}{X_{i}}\left\|U_{1}\right\|_{X} \cdots\left\|U_{r}\right\|_{X}\|V\|_{X},
$$

where $C$ is the same constant as in (4.3). 
Proof. Let $U_{\mu}(x)=\sum_{\left|\alpha^{\mu}\right| \geq 1} U_{\alpha^{\mu}}^{\mu} x^{\alpha^{\mu}} \in \mathcal{O}_{0}(X)(\mu=1, \ldots, r)$ and $V(x)=$ $\sum_{|\beta| \geq 1} V_{\beta} x^{\beta} \in \mathcal{O}_{0}(X)$. Then is holds that

$$
\begin{aligned}
x^{\alpha}\left(\Lambda^{-1} U_{1}\right) \cdots\left(\Lambda^{-1} U_{r}\right) \partial_{x_{i}} \Lambda^{-1} V & \\
=\sum_{\left|\alpha^{1}\right| \geq 1, \ldots,\left|\alpha^{r}\right| \geq 1,|\beta| \geq 1} \frac{1}{\lambda \cdot \alpha^{1}-f_{u}(0,0)} & \cdots \frac{1}{\lambda \cdot \alpha^{r}-f_{u}(0,0)} \frac{\beta_{i}}{\lambda \cdot \beta-f_{u}(0,0)} \\
& \times U_{\alpha^{1}} \cdots U_{\alpha^{r}} V_{\beta} x^{\alpha+\alpha^{1}+\cdots+\alpha^{r}+\beta-\mathrm{e}_{i}}
\end{aligned}
$$

which implies

$$
\begin{aligned}
& \left\|x^{\alpha}\left(\Lambda^{-1} U_{1}\right) \cdots\left(\Lambda^{-1} U_{r}\right) \partial_{x_{i}} \Lambda^{-1} V\right\|_{X} \\
& =\sum_{\left|\alpha^{1}\right| \geq 1, \ldots,\left|\alpha^{r}\right| \geq 1,|\beta| \geq 1} \frac{1}{\left|\lambda \cdot \alpha^{1}-f_{u}(0,0)\right|} \cdots \frac{1}{\left|\lambda \cdot \alpha^{r}-f_{u}(0,0)\right|} \\
& \quad \times \frac{\left|\beta_{i}\right|}{\left|\lambda \cdot \beta-f_{u}(0,0)\right|}\left|U_{\alpha^{1}}\right| \cdots\left|U_{\alpha^{r}}\right|\left|V_{\beta}\right| X^{\alpha+\alpha^{1}+\cdots+\alpha^{r}+\beta-\mathrm{e}_{i}} .
\end{aligned}
$$

By (4.3) and (4.6) we obtain (4.5).

Lemma 4.2. Let us assume that $|\alpha|+r \geq 1$, and let $U$ and $V$ belong to $\mathcal{O}_{0}(X)$. Then it holds that

$$
\begin{gathered}
\left\|x^{\alpha}\left(\Lambda^{-1} U\right)^{r} \partial_{x_{i}} \Lambda^{-1} U-x^{\alpha}\left(\Lambda^{-1} V\right)^{r} \partial_{x_{i}} \Lambda^{-1} V\right\|_{X} \\
\leq C^{r+1} \frac{X^{\alpha}}{X_{i}}\|U-V\|_{X} \times \sum_{\nu=0}^{r}\|U\|_{X}^{r-\nu}\|V\|_{X}{ }^{\nu} .
\end{gathered}
$$

Proof. It holds that

$$
\begin{aligned}
& x^{\alpha}\left(\Lambda^{-1} U\right)^{r} \partial_{x_{i}} \Lambda^{-1} U-x^{\alpha}\left(\Lambda^{-1} V\right)^{r} \partial_{x_{i}} \Lambda^{-1} V \\
&=x^{\alpha}\left[\left\{\left(\Lambda^{-1} U\right)^{r}-\left(\Lambda^{-1} V\right)^{r}\right\} \partial_{x_{i}} \Lambda^{-1} U+\left(\Lambda^{-1} V\right)^{r} \partial_{x_{i}} \Lambda^{-1}(U-V)\right] \\
&=x^{\alpha}\left[\left\{\Lambda^{-1}(U-V)\right\} \sum_{\nu=0}^{r-1}\left(\Lambda^{-1} U\right)^{r-1-\nu}\left(\Lambda^{-1} V\right)^{\nu} \partial_{x_{i}} \Lambda^{-1} U\right. \\
&\left.+\left(\Lambda^{-1} V\right)^{r} \partial_{x_{i}} \Lambda^{-1}(U-V)\right] .
\end{aligned}
$$

By Lemma 4.1 and (4.8) we obtain (4.7).

By Lemmas 3.7, (1), 4.1, 4.2 and (4.4) we have the following. 
Lemma 4.3. Let us assume that $|\alpha|+r \geq 1$, and let $U$ and $V$ belong to $\mathcal{O}_{0}(X)$. Let $\mathcal{F}(x, u)$ be holomorphic at $(x, u)=(0,0)$. Then it holds that

$$
\begin{aligned}
&\left\|\mathcal{F}\left(x, \Lambda^{-1} U\right) x^{\alpha}\left(\Lambda^{-1} U\right)^{r} \partial_{x_{i}} \Lambda^{-1} U-\mathcal{F}\left(x, \Lambda^{-1} V\right) x^{\alpha}\left(\Lambda^{-1} V\right)^{r} \partial_{x_{i}} \Lambda^{-1} V\right\|_{X} \\
& \leq C^{r+1} \frac{X^{\alpha}}{X_{i}}\|U-V\|_{X} \times\left\{C\left|\mathcal{F}_{u}\right|\left(X, C\left(\|U\|_{X}+\|V\|_{X}\right)\right) \cdot\|U\|^{r+1}\right. \\
&\left.+|\mathcal{F}|\left(X, C\|V\|_{X}\right) \cdot \sum_{\nu=0}^{r}\|U\|_{X}{ }^{r-\nu}\|V\|_{X}{ }^{\nu}\right\} .
\end{aligned}
$$

Proof of Case (iv) of Theorem 2.1 (continued). If $U(x) \in \mathcal{O}_{0}(X)$, then it follows from Lemmas 3.2, $(1)^{\prime}, 3.4,(1), 3.5,(1), 4.1$ and $(4.4)$ that $T U(x) \in$ $\mathcal{O}_{0}(X)$ and that

$$
\begin{aligned}
\|T U\|_{X} \leq & C \sum_{i=1}^{m-1} \frac{X_{i+1}}{X_{i}}\|U\|_{X} \\
& +\sum_{i=1}^{m} \frac{1}{X_{i}}\left(\sum_{|\alpha|+r \geq 2}^{\text {finite }}\left|c_{i \alpha r}\right|\left(X, C\|U\|_{X}\right) X^{\alpha}\left(C\|U\|_{X}\right)^{r+1}\right) \\
& +\left|g_{0}\right|(X)+|g|\left(X, C\|U\|_{X}\right) \\
\equiv & A\left(X,\|U\|_{X}\right) .
\end{aligned}
$$

By the expression of $A\left(X,\|U\|_{X}\right)$ we can take $X$ and $\varepsilon$ such that $A(X, \varepsilon) \leq \varepsilon$. This shows that the operator $T: \mathcal{O}_{0}(X ; \varepsilon) \rightarrow \mathcal{O}_{0}(X ; \varepsilon)$ is well-defined.

Furthermore it follows from Lemmas 3.6, (1), 4.3 and (4.4) that

$$
\begin{aligned}
& \|T U-T V\|_{X} \\
& \leq\|U-V\|_{X} \\
& \times C\left[\sum_{i=1}^{m-1} \frac{X_{i+1}}{X_{i}}+\sum_{i=1}^{m} \frac{1}{X_{i}}\left(\sum_{|\alpha|+r \geq 2}^{\text {finite }}\left|c_{i \alpha r, u}\right|\left(X, C\left(\|U\|_{X}+\|V\|_{X}\right)\right)\right.\right. \\
& \left.\times X^{\alpha}\left(C\|U\|_{X}\right)^{r+1}\right) \\
& +\sum_{i=1}^{m} \frac{1}{X_{i}}\left(\sum_{|\alpha|+r \geq 2}^{\text {finite }}\left|c_{i \alpha r}\right|\left(X, C\|V\|_{X}\right) X^{\alpha} C^{r} \cdot \sum_{\nu=0}^{r}\|U\|_{X}{ }^{r-\nu}\|V\|_{X}{ }^{\nu}\right) \\
& \left.+\left|g_{u}\right|\left(X, C\left(\|U\|_{X}+\|V\|_{X}\right)\right)\right]
\end{aligned}
$$




$$
\equiv\|U-V\|_{X} \times \widetilde{A}\left(X,\|U\|_{X},\|V\|_{X}\right)
$$

By the expression of $\widetilde{A}\left(X,\|U\|_{X},\|V\|_{X}\right)$ we can take $X$ and $\varepsilon$ such that $\widetilde{A}(X, \varepsilon$, $\varepsilon)<1$. This shows that $T: \mathcal{O}_{0}(X ; \varepsilon) \rightarrow \mathcal{O}_{0}(X ; \varepsilon)$ is a contraction mapping. Therefore there exists a unique $U(x) \in \mathcal{O}_{0}(X ; \varepsilon)$ which satisfies (4.2). Lemma 3.1 implies $U(x) \in G^{\left\{1^{(d)}\right\}}$. Hence $u(x)=\Lambda^{-1} U(x)$ also belongs to $G^{\left\{1^{(d)}\right\}}$ and it is a solution of (2.5). Since we admit the unique existence of the formal solution, the proof is completed.

\section{§5. Proof of Theorem 2.1 (Cases (v), (vi), (vii))}

In this section we study Cases (v), (vi) and (vii). We admit the unique existence of the formal solution, and prove its Gevrey order. As mentioned in $§ 2$, we only consider Case (v), that is, we only consider the equation (2.6). Furthermore, for simplicity we assume $k=1$. We write a formal power series solution as $u(y, z)=\sum_{(\beta, \gamma) \in \mathbf{N}^{n+p},|\beta|+|\gamma| \geq 1} u_{\beta \gamma} y^{\beta} z^{\gamma}(n+p=d)$ and use the Banach space $G_{0, k}^{\{\sigma, \tau\}}(Y, Z)\left(Y=\left(Y_{1}, \ldots, Y_{n}\right) \in\left(\mathbf{R}_{+} \backslash\{0\}\right)^{n}, Z=\left(Z_{1}, \ldots, Z_{p}\right) \in\left(\mathbf{R}_{+} \backslash\{0\}\right)^{p}\right)$. Therefore $u(y, z) \in G_{0, k}^{\{\sigma, \tau\}}(Y, Z)$ means

$$
\|u\|_{k, Y, Z}^{\{\sigma, \tau\}} \equiv \sum_{|\beta|+|\gamma| \geq 1}\left|u_{\beta \gamma}\right| \frac{(|\beta|+|\gamma|) !}{(\sigma \cdot \beta+\tau \cdot \gamma-k) !} Y^{\beta} Z^{\gamma}<+\infty .
$$

In the equation (2.6) we may assume that $f_{u}(0,0)=1$ since $f_{u}(0,0) \neq 0$. Hence (2.6) is written as follows when $k=1$ :

$$
u(y, z)=T u(y, z) \equiv P^{\prime \prime \prime} u(y, z)+g_{0}(y, z)+g(y, z, u(y, z)), \quad u(0,0)=0
$$

where $g_{0}$ and $g$ are holomorphic at the origin which satisfy $g_{0}(0,0)=0$ and $g(y, z, 0) \equiv 0, g_{u}(0,0,0)=0$, respectively, and

$$
\begin{aligned}
P^{\prime \prime \prime} u= & \sum_{j=1}^{n-1} y_{j+1} \frac{\partial u}{\partial y_{j}}+\sum_{j=1}^{n}\left(\sum_{|\beta|+|\gamma|+r \geq 2}^{\text {finite }} d_{j \beta \gamma r}(y, z, u) y^{\beta} z^{\gamma} u^{r}\right) \frac{\partial u}{\partial y_{j}} \\
& +\sum_{q=1}^{p}\left(\sum_{|\beta|+|\gamma|+r \geq 2}^{\text {finite }} e_{q \beta \gamma r}(y, z, u) y^{\beta} z^{\gamma} u^{r}\right) \frac{\partial u}{\partial z_{q}}
\end{aligned}
$$

Here all coefficients $d_{j \beta \gamma r}$ and $e_{q \beta \gamma r}$ are holomorphic at the origin, and none of them vanish at the origin unless they vanish identically.

We assume that $s=(\sigma, \tau)$ satisfies the condition in Theorem 2.1, and prove that the formal solution of (5.1) belongs to $G^{\{\sigma, \tau\}}$. 
Proof of Case (v) of Theorem 2.1. Let us write the $\varepsilon$-closed ball in $G_{0, k}^{\{\sigma, \tau\}}(Y, Z)$ as

$$
\begin{aligned}
G_{0, k}^{\{\sigma, \tau\}} & (Y, Z ; \varepsilon) \\
& =\left\{u(y, z)=\sum_{|\beta|+|\gamma| \geq 1} u_{\beta \gamma} y^{\beta} z^{\gamma} \in G_{0, k}^{\{\sigma, \tau\}}(Y, Z) ;\|u\|_{k, Y, Z}^{\{\sigma, \tau\}} \leq \varepsilon\right\} .
\end{aligned}
$$

We shall prove that the operator $T$ is well-defined as the mapping from $G_{0, k}^{\{\sigma, \tau\}}(Y, Z ; \varepsilon)$ to itself by choosing $k, X, Y$ and $\varepsilon$ suitably and that it becomes a contraction mapping there.

Here we give some lemmas.

Lemma 5.1. Let $|\beta|+|\gamma|+r \geq 1$ and $\left|\beta^{\prime}\right|+\left|\gamma^{\prime}\right| \geq 1$. Let us assume that $(\sigma, \tau) \in[1,+\infty)^{d}$ satisfies

$$
\begin{gathered}
\sigma \cdot\left(\beta+r \mathrm{e}_{j}^{(n)}-\beta^{\prime}\right)+\tau \cdot\left(\gamma-\gamma^{\prime}\right) \geq|\beta|+|\gamma|+r \quad(j=1, \ldots, n), \\
\sigma \cdot\left(\beta-\beta^{\prime}\right)+\tau \cdot\left(\gamma+r \mathrm{e}_{q}^{(p)}-\gamma^{\prime}\right) \geq|\beta|+|\gamma|+r \quad(q=1, \ldots, p),
\end{gathered}
$$

where $\mathrm{e}_{j}^{(n)}=\left(\delta_{j 1}, \ldots, \delta_{j n}\right)(j=1, \ldots, n)$ and $\mathrm{e}_{q}^{(p)}=\left(\delta_{q 1}, \ldots, \delta_{q p}\right)(q=1, \ldots, p)$. If $k$ satisfies

$$
\begin{aligned}
& \frac{(1-\sigma) \cdot \beta+(1-\tau) \cdot \gamma+\sigma \cdot \beta^{\prime}+\tau \cdot \gamma^{\prime}}{r} \\
& \quad \leq k \leq \min \left\{\sigma_{1}-1, \ldots, \sigma_{n}-1, \tau_{1}-1, \ldots, \tau_{p}-1\right\},
\end{aligned}
$$

and $u_{1}, \ldots, u_{r}, v \in G_{0, k}^{\{\sigma, \tau\}}(Y, Z)$, then $y^{\beta} z^{\gamma} u_{1} \cdots u_{r} D_{y}^{\beta^{\prime}} D_{z}^{\gamma^{\prime}} v$ belongs to $G_{0, k}^{\{\sigma, \tau\}}(Y, Z)$ and it holds that

$$
\begin{aligned}
& \left\|y^{\beta} z^{\gamma} u_{1} \cdots u_{r} D_{y}^{\beta^{\prime}} D_{z}^{\gamma^{\prime}} v\right\|_{k, Y, Z}^{\{\sigma, \tau\}} \\
& \quad \leq \mathcal{C}^{r-|\beta|-|\gamma|} \frac{Y^{\beta}}{Y^{\beta^{\prime}}} \frac{Z^{\gamma}}{Z^{\gamma^{\prime}}}\left\|u_{1}\right\|_{k, Y, Z}^{\{\sigma, \tau\}} \cdots\left\|u_{r}\right\|_{k, Y, Z}^{\{\sigma, \tau\}}\|v\|_{k, Y, Z}^{\{\sigma, \tau\}},
\end{aligned}
$$

where

$$
\mathcal{C}= \begin{cases}1 & (\text { when }|\beta|+|\gamma| \geq r) \\ \max \left\{\sigma_{1}, \ldots, \sigma_{n}, \tau_{1}, \ldots, \tau_{p}\right\} & (\text { when }|\beta|+|\gamma| \leq r-1)\end{cases}
$$

Remark 5.1. The condition (5.2) implies

$$
\begin{aligned}
& \frac{(1-\sigma) \cdot \beta+(1-\tau) \cdot \gamma+\sigma \cdot \beta^{\prime}+\tau \cdot \gamma^{\prime}}{r} \\
& \quad \leq \min \left\{\sigma_{1}-1, \ldots, \sigma_{n}-1, \tau_{1}-1, \ldots, \tau_{p}-1\right\}
\end{aligned}
$$


Remark 5.2. Let us write the Newton polyhedron of $y^{\beta} z^{\gamma} u(y, z)^{r}$ $\times D_{y}{ }^{\beta^{\prime}} D_{z}{ }^{\gamma^{\prime}} u(y, z)$ as

$$
\begin{aligned}
& N\left(y^{\beta} z^{\gamma} u(y, z)^{r} D_{y}{ }^{\beta^{\prime}} D_{z}^{\gamma^{\prime}} u(y, z)\right) \\
& \quad=\operatorname{Ch}\left\{\bigcup_{j=1}^{n} Q\left(\beta+r \mathrm{e}_{j}^{(n)}, \gamma, \beta^{\prime}, \gamma^{\prime}\right) \cup \bigcup_{q=1}^{p} Q\left(\beta, \gamma+r \mathrm{e}_{q}^{(p)}, \beta^{\prime}, \gamma^{\prime}\right)\right\},
\end{aligned}
$$

where

$$
Q\left(\beta, \gamma, \beta^{\prime}, \gamma^{\prime}\right)=\left\{\begin{array}{ll} 
& \mathcal{Y}_{j} \geq \beta_{j}-\beta^{\prime}{ }_{j} \quad(j=1, \ldots, n) \\
(\mathcal{Y}, \mathcal{Z}, \mathcal{W}) \in \mathbf{R}^{d+1} ; & \mathcal{Z}_{q} \geq \gamma_{q}-\gamma^{\prime}{ }_{q} \\
& \mathcal{W} \leq\left|\beta^{\prime}\right|+\left|\gamma^{\prime}\right|
\end{array}\right\}
$$

Furthermore we define $\Pi(\sigma, \tau)$ by

$$
\Pi(\sigma, \tau)=\left\{(\mathcal{Y}, \mathcal{Z}, \mathcal{W}) \in \mathbf{R}^{d+1} ;\left(\sigma-1^{(n)}\right) \cdot \mathcal{Y}+\left(\tau-1^{(p)}\right) \cdot \mathcal{Z}-\mathcal{W} \geq 0\right\}
$$

and define $S$ by

$$
S=\left\{(\sigma, \tau) \in[1,+\infty)^{d} ; N\left(y^{\beta} z^{\gamma} u(y, z)^{r} D_{y}{ }^{\beta^{\prime}} D_{z}{ }^{\gamma^{\prime}} u(y, z)\right) \subset \Pi(\sigma, \tau)\right\} .
$$

Then the condition $(\sigma, \tau) \in S$ is equivalent to (5.2).

Proof of Lemma 5.1. Let $u_{\mu}(y, z)=\sum_{\left|\beta^{\mu}\right|+\left|\gamma^{\mu}\right| \geq 1} u_{\beta^{\mu} \gamma^{\mu}}^{\mu} y^{\beta^{\mu}} z^{\gamma^{\mu}} \in$ $G_{0, k}^{\{\sigma, \tau\}}(Y, Z)(\mu=1, \ldots, n)$ and $v(y, z)=\sum_{|\widetilde{\beta}|+|\widetilde{\gamma}| \geq 1} v_{\widetilde{\beta} \widetilde{\gamma}} y^{\widetilde{\beta}} z^{\widetilde{\gamma}} \in G_{0, k}^{\{\sigma, \tau\}}(Y, Z)$. Then it holds that

$$
\begin{aligned}
& y^{\beta} z^{\gamma} u_{1}(y, z) \cdots u_{r}(y, z) D_{y}^{\beta^{\prime}} D_{z}^{\gamma^{\prime}} v(y, z) \\
& =\sum_{\substack{\left|\beta^{1}\right|+\left|\gamma^{1}\right| \geq 1, \ldots,\left|\beta^{r}\right|+\left|\gamma^{r}\right| \geq 1 \\
\tilde{\beta} \geq \beta^{\prime}, \tilde{\gamma} \geq \gamma^{\prime}}} u_{\beta^{1} \gamma^{1}}^{1} \cdots u_{\beta^{r} \gamma^{r}}^{r} v_{\widetilde{\beta} \widetilde{\gamma}} \\
& \\
& \quad \times \frac{\widetilde{\beta} !}{\left(\widetilde{\beta}-\beta^{\prime}\right) !} \frac{\widetilde{\gamma} !}{\left(\widetilde{\gamma}-\gamma^{\prime}\right) !} y^{\beta+\beta^{1}+\cdots+\beta^{r}+\widetilde{\beta}-\beta^{\prime}} z^{\gamma+\gamma^{1}+\cdots+\gamma^{r}+\widetilde{\gamma}-\gamma^{\prime}},
\end{aligned}
$$

which implies that

$$
\begin{aligned}
& \left\|y^{\beta} z^{\gamma} u_{1} \cdots u_{r} D_{y}{ }^{\beta^{\prime}} D_{z^{\gamma^{\prime}}}^{\gamma^{\prime}} v\right\|_{k, Y, Z}^{\{\sigma, \tau\}} \\
& =\sum_{\substack{\left|\beta^{1}\right|+\left|\gamma^{1}\right| \geq 1, \ldots,\left|\beta^{r}\right|+\left|\gamma^{r}\right| \geq 1 \\
\widetilde{\beta} \geq \beta^{\prime}, \tilde{\gamma} \geq \gamma^{\prime}}}\left|u_{\beta^{1} \gamma^{1}}^{1}\right| \cdots\left|u_{\beta^{r} \gamma^{r}}^{r}\right|\left|v_{\widetilde{\beta} \widetilde{\gamma}}\right| \frac{\widetilde{\beta} !}{\left(\widetilde{\beta}-\beta^{\prime}\right) !} \frac{\widetilde{\gamma} !}{\left(\widetilde{\gamma}-\gamma^{\prime}\right) !} \\
& \quad \times \frac{\left(\left|\beta+\bar{\beta}+\widetilde{\beta}-\beta^{\prime}\right|+\left|\gamma+\bar{\gamma}+\widetilde{\gamma}-\gamma^{\prime}\right|\right) !}{\left\{\sigma \cdot\left(\beta+\bar{\beta}+\widetilde{\beta}-\beta^{\prime}\right)+\tau \cdot\left(\gamma+\bar{\gamma}+\widetilde{\gamma}-\gamma^{\prime}\right)-k\right\} !}
\end{aligned}
$$




$$
\times Y^{\beta+\bar{\beta}+\widetilde{\beta}-\beta^{\prime}} Z^{\gamma+\bar{\gamma}+\widetilde{\gamma}-\gamma^{\prime}}
$$

where $\bar{\beta}=\beta^{1}+\cdots+\beta^{r}, \bar{\gamma}=\gamma^{1}+\cdots+\gamma^{r}$.

Therefore it is sufficient to prove the following inequality:

$$
\begin{gathered}
\frac{\widetilde{\beta} !}{\left(\widetilde{\beta}-\beta^{\prime}\right) !} \frac{\widetilde{\gamma} !}{\left(\widetilde{\gamma}-\gamma^{\prime}\right) !} \frac{\left\{\prod_{\mu=1}^{r}\left(\sigma \cdot \beta^{\mu}+\tau \cdot \gamma^{\mu}-k\right) !\right\}(\sigma \cdot \widetilde{\beta}+\tau \cdot \widetilde{\gamma}-k) !}{\left\{\sigma \cdot\left(\beta+\bar{\beta}+\widetilde{\beta}-\beta^{\prime}\right)+\tau \cdot\left(\gamma+\bar{\gamma}+\widetilde{\gamma}-\gamma^{\prime}\right)-k\right\} !} \\
\leq \mathcal{C}^{r-|\beta|-|\gamma|} \frac{\left\{\prod_{\mu=1}^{r}\left(\left|\beta^{\mu}\right|+\left|\gamma^{\mu}\right|\right) !\right\}(|\widetilde{\beta}|+|\widetilde{\gamma}|) !}{\left(\left|\beta+\bar{\beta}+\widetilde{\beta}-\beta^{\prime}\right|+\left|\gamma+\bar{\gamma}+\widetilde{\gamma}-\gamma^{\prime}\right|\right) !} \\
\left(\left|\beta^{1}\right|+\left|\gamma^{1}\right| \geq 1, \ldots,\left|\beta^{r}\right|+\left|\gamma^{r}\right| \geq 1, \widetilde{\beta} \geq \beta^{\prime}, \widetilde{\gamma} \geq \gamma^{\prime}\right) .
\end{gathered}
$$

Let us prove $(5.6) . r k \geq(1-\sigma) \cdot \beta+(1-\tau) \cdot \gamma+\sigma \cdot \beta^{\prime}+\tau \cdot \gamma^{\prime}$ implies

$$
\begin{aligned}
& \quad \frac{\left\{\prod_{\mu=1}^{r}\left(\sigma \cdot \beta^{\mu}+\tau \cdot \gamma^{\mu}-k\right) !\right\}(\sigma \cdot \widetilde{\beta}+\tau \cdot \widetilde{\gamma}-k) !}{\left\{\sigma \cdot\left(\beta+\bar{\beta}+\widetilde{\beta}-\beta^{\prime}\right)+\tau \cdot\left(\gamma+\bar{\gamma}+\widetilde{\gamma}-\gamma^{\prime}\right)-k\right\} !} \\
& \quad=\frac{\left\{\prod_{\mu=1}^{r} \Gamma\left(\sigma \cdot \beta^{\mu}+\tau \cdot \gamma^{\mu}-k+1\right)\right\} \Gamma(\sigma \cdot \widetilde{\beta}+\tau \cdot \widetilde{\gamma}-k+1)}{\Gamma\left(\sigma \cdot(\bar{\beta}+\widetilde{\beta})+\tau \cdot(\bar{\gamma}+\widetilde{\gamma})+\sigma \cdot\left(\beta-\beta^{\prime}\right)+\tau \cdot\left(\gamma-\gamma^{\prime}\right)-k+1\right)} \\
& \quad \leq \frac{\left\{\prod_{\mu=1}^{r} \Gamma\left(\sigma \cdot \beta^{\mu}+\tau \cdot \gamma^{\nu}-k+1\right)\right\} \Gamma(\sigma \cdot \widetilde{\beta}+\tau \cdot \widetilde{\gamma}-k+1)}{\Gamma(K+|\beta|+|\gamma|+1)} \equiv \boldsymbol{\Gamma}
\end{aligned}
$$

where

$$
K=\sigma \cdot(\bar{\beta}+\widetilde{\beta})+\tau \cdot(\bar{\gamma}+\widetilde{\gamma})-(r+1) k
$$

Here we remark the following fact: Dirichlet's integral

$$
\begin{aligned}
\mathcal{D} & \left.\eta_{0}, \eta_{1}, \ldots, \eta_{r}\right) \\
& =\frac{\Gamma\left(\eta_{0}\right) \Gamma\left(\eta_{1}\right) \cdots \Gamma\left(\eta_{r}\right)}{\Gamma\left(\eta_{0}+\eta_{1}+\cdots+\eta_{r}\right)} \\
& =\int \cdots \int_{\substack{t_{1} \geq 0, \ldots, t_{r} \geq 0 \\
t_{1}+\cdots+t_{r} \leq 1}}\left(1-\left(t_{1}+\cdots+t_{r}\right)\right)^{\eta_{0}-1} t_{1} \eta_{1}-1 \cdots t_{r} \eta_{r}-1 d t_{1} \cdots d t_{r}
\end{aligned}
$$

satisfies

$$
0<\eta_{i}<\zeta_{i}(i=0,1, \ldots, r) \Longrightarrow \mathcal{D}\left(\eta_{0}, \eta_{1}, \ldots, \eta_{r}\right)>\mathcal{D}\left(\zeta_{0}, \zeta_{1}, \ldots, \zeta_{r}\right)
$$

Case (I): $|\beta|+|\gamma| \geq r+1$ : In this case it holds that

$$
\Gamma(K+|\beta|+|\gamma|+1)=\Gamma(K+r+1) \cdot \prod_{l=r+1}^{|\beta|+|\gamma|}(K+l),
$$


which implies

$$
\begin{aligned}
\boldsymbol{\Gamma}= & \frac{\left\{\prod_{\mu=1}^{r} \Gamma\left(\sigma \cdot \beta^{\mu}+\tau \cdot \gamma^{\mu}-k+1\right)\right\} \Gamma(\sigma \cdot \widetilde{\beta}+\tau \cdot \widetilde{\gamma}-k+1)}{\Gamma(K+r+1)} \frac{1}{\prod_{l=r+1}^{|\beta|+|\gamma|}(K+l)} \\
= & \mathcal{D}\left(\sigma \cdot \beta^{1}+\tau \cdot \gamma^{1}-k+1, \ldots, \sigma \cdot \beta^{r}+\tau \cdot \gamma^{r}-k+1, \sigma \cdot \widetilde{\beta}+\tau \cdot \widetilde{\gamma}-k+1\right) \\
& \times \frac{1}{\prod_{l=r+1}^{|\beta|+|\gamma|}(K+l)} \\
\leq & \mathcal{D}\left(\left|\beta^{1}\right|+\left|\gamma^{1}\right|+1, \ldots,\left|\beta^{r}\right|+\left|\gamma^{r}\right|+1,|\widetilde{\beta}|+|\widetilde{\gamma}|+1\right) \frac{1}{\prod_{l=r+1}^{|\beta|+|\gamma|}(K+l)} \\
& \left(\operatorname{since} k \leq \min \left\{\sigma_{1}-1, \ldots, \sigma_{n}-1, \tau_{1}-1, \ldots, \tau_{p}-1\right\}\right) \\
= & \frac{\left\{\prod_{\mu=1}^{r}\left(\left|\beta^{\mu}\right|+\left|\gamma^{\mu}\right|\right) !\right\}(|\widetilde{\beta}|+|\widetilde{\gamma}|) !}{(|\bar{\beta}+\widetilde{\beta}|+|\bar{\gamma}+\widetilde{\gamma}|+r) !} \frac{1}{\prod_{l=r+1}^{|\beta|+|\gamma|}(K+l)} \\
= & \frac{\left\{\prod_{\mu=1}^{r}\left(\left|\beta^{\mu}\right|+\left|\gamma^{\mu}\right|\right) !\right\}(|\widetilde{\beta}|+|\widetilde{\gamma}|) !}{\widetilde{K} !} \frac{1}{\prod_{l=1}^{r}(\widetilde{K}+l)} \frac{1}{\prod_{l=r+1}^{|\beta|+|\gamma|}(K+l)}
\end{aligned}
$$

where

$$
\widetilde{K}=|\bar{\beta}+\widetilde{\beta}|+|\bar{\gamma}+\widetilde{\gamma}| .
$$

Moreover it follows from $K \geq \widetilde{K}$ that

$$
\begin{aligned}
& \frac{\widetilde{\beta} !}{\left(\widetilde{\beta}-\beta^{\prime}\right) !} \frac{\widetilde{\gamma} !}{\left(\widetilde{\gamma}-\gamma^{\prime}\right) !} \frac{\left(\widetilde{K}+\left|\beta-\beta^{\prime}\right|+\left|\gamma-\gamma^{\prime}\right|\right) !}{\widetilde{K} !} \frac{1}{\prod_{l=1}^{r}(\widetilde{K}+l)} \frac{1}{\prod_{l=r+1}^{|\beta|+|\gamma|}(K+l)} \\
& \quad \leq \frac{\widetilde{\beta} !}{\left(\widetilde{\beta}-\beta^{\prime}\right) !} \frac{\widetilde{\gamma} !}{\left(\widetilde{\gamma}-\gamma^{\prime}\right) !} \frac{\left(\widetilde{K}-\left|\beta^{\prime}\right|-\left|\gamma^{\prime}\right|\right) !}{\widetilde{K} !} \prod_{l=1}^{|\beta|+|\gamma|} \frac{\widetilde{K}-\left|\beta^{\prime}\right|-\left|\gamma^{\prime}\right|+l}{\widetilde{K}+l} \\
& \quad \leq 1 .
\end{aligned}
$$

By the above inequalities we obtain (5.6).

Case (II): $|\beta|+|\gamma|=r$ : In this case it holds that

$$
\begin{aligned}
\boldsymbol{\Gamma} & =\frac{\left\{\prod_{\mu=1}^{r} \Gamma\left(\sigma \cdot \beta^{\mu}+\tau \cdot \gamma^{\mu}-k+1\right)\right\} \Gamma(\sigma \cdot \widetilde{\beta}+\tau \cdot \widetilde{\gamma}-k+1)}{\Gamma(K+r+1)} \\
& =\mathcal{D}\left(\sigma \cdot \beta^{1}+\tau \cdot \gamma^{1}-k+1, \ldots, \sigma \cdot \beta^{r}+\tau \cdot \gamma^{r}-k+1, \sigma \cdot \widetilde{\beta}+\tau \cdot \widetilde{\gamma}-k+1\right) \\
& \leq \mathcal{D}\left(\left|\beta^{1}\right|+\left|\gamma^{1}\right|+1, \ldots,\left|\beta^{r}\right|+\left|\gamma^{r}\right|+1,|\widetilde{\beta}|+|\widetilde{\gamma}|+1\right) \\
& =\frac{\left\{\prod_{\mu=1}^{r}\left(\left|\beta^{\mu}\right|+\left|\gamma^{\mu}\right|\right) !\right\}(|\widetilde{\beta}|+|\widetilde{\gamma}|) !}{\widetilde{K} !} \frac{1}{\prod_{l=1}^{|\beta|+|\gamma|}(\widetilde{K}+l)} .
\end{aligned}
$$


From this inequality and (5.7) we obtain (5.6).

Case (III): $|\beta|+|\gamma| \leq r-1$ : In this case it holds that

$$
\Gamma(K+r+1)=\Gamma(K+|\beta|+|\gamma|+1) \prod_{l=|\beta|+|\gamma|+1}^{r}(K+l)
$$

which implies

$$
\begin{aligned}
\boldsymbol{\Gamma} & =\frac{\left\{\prod_{\mu=1}^{r} \Gamma\left(\sigma \cdot \beta^{\mu}+\tau \cdot \gamma^{\mu}-k+1\right)\right\} \Gamma(\sigma \cdot \widetilde{\beta}+\tau \cdot \widetilde{\gamma}-k+1)}{\Gamma(K+r+1)} \prod_{l=|\beta|+|\gamma|+1}^{r}(K+l) \\
& \leq \frac{\left\{\prod_{\mu=1}^{r}\left(\left|\beta^{\mu}\right|+\left|\gamma^{\mu}\right|\right) !\right\}(|\widetilde{\beta}|+|\widetilde{\gamma}|) !}{(\widetilde{K}+r) !} \prod_{l=|\beta|+|\gamma|+1}^{r}(K+l) \\
& =\frac{\left\{\prod_{\mu=1}^{r}\left(\left|\beta^{\mu}\right|+\left|\gamma^{\mu}\right|\right) !\right\}(|\widetilde{\beta}|+|\widetilde{\gamma}|) !}{\widetilde{K} !} \frac{1}{\prod_{l=1}^{|\beta|+|\gamma|}(\widetilde{K}+l)} \prod_{l=|\beta|+|\gamma|+1}^{r} \frac{K+l}{\widetilde{K}+l} \\
& \leq \mathcal{C}^{r-|\beta|-|\gamma|} \frac{\left\{\prod_{\mu=1}^{r}\left(\left|\beta^{\mu}\right|+\left|\gamma^{\mu}\right|\right) !\right\}(|\widetilde{\beta}|+|\widetilde{\gamma}|) !}{\widetilde{K} !} \frac{1}{\prod_{l=1}^{|\beta|+|\gamma|}(\widetilde{K}+l)} .
\end{aligned}
$$

From this inequality and (5.7) we obtain (5.6).

The proof is completed.

From Lemma 5.1, similarly to the proof of Lemma 4.3 we can prove the following lemma.

Lemma 5.2. Let $|\beta|+|\gamma|+r \geq 1,\left|\beta^{\prime}\right|+\left|\gamma^{\prime}\right| \geq 1$, and let us assume that $(\sigma, \tau) \in[1,+\infty)^{d}$ and $k$ satisfy (5.2) and (5.3), respectively. Let $u(y, z)$ and $v(y, z)$ belong to $G_{0, k}^{\{\sigma, \tau\}}(Y, Z)$, and let $\mathcal{F}(x, y, u)$ be holomorphic at $(x, y, u)=$ $(0,0,0)$. Then it holds that

$$
\begin{aligned}
& \left\|\mathcal{F}(x, y, u) y^{\beta} z^{\gamma} u^{r} D_{y}^{\beta^{\prime}} D_{z}^{\gamma^{\prime}} u-\mathcal{F}(x, y, v) y^{\beta} z^{\gamma} v^{r} D_{y}^{\beta^{\prime}} D_{z}^{\gamma^{\prime}} v\right\|_{k, Y, Z}^{\{\sigma, \tau\}} \\
& \leq \mathcal{C}^{r-|\beta|-|\gamma|} \frac{Y^{\beta}}{Y^{\beta^{\prime}}} \frac{Z^{\gamma}}{Z^{\gamma^{\prime}}}\|u-v\|_{k, Y, Z}^{\{\sigma, \tau\}} \\
& \quad \times\left\{\mathrm{S}\left|\mathcal{F}_{u}\right|\left(Y, Z, \mathrm{~S}\left(\|u\|_{k, Y, Z}^{\{\sigma, \tau\}}+\|v\|_{k, Y, Z}^{\{\sigma, \tau\}}\right)\right) \cdot\left(\|u\|_{k, Y, Z}^{\{\sigma, \tau\}}\right)^{r+1}\right. \\
& \left.\quad+|\mathcal{F}|\left(Y, Z, \mathrm{~S}\|v\|_{k, Y, Z}^{\{\sigma, \tau\}}\right) \cdot \sum_{\nu=0}^{r}\left(\|u\|_{k, Y, Z}^{\{\sigma, \tau\}}\right)^{r-\nu}\left(\|v\|_{k, Y, Z}^{\{\sigma, \tau\}}\right)^{\nu}\right\}
\end{aligned}
$$

where $\mathcal{C}$ is same as in $(5.5)$ and $\mathbf{S}=\max \left\{\sigma_{1}, \ldots, \sigma_{n}, \tau_{1}, \ldots, \tau_{p}\right\}$ 
Proof of Case (v) of Theorem 2.1 (continued). Put $k_{0}=\min \left\{\sigma_{1}-1, \ldots\right.$, $\left.\sigma_{n}-1, \tau_{1}-1, \ldots, \tau_{p}-1\right\}$, and let $u(y, z) \in G_{0, k_{0}}^{\{\sigma, \tau\}}(Y, Z)$. Then it follows from the assumption $(\sigma, \tau) \in S_{5}$ and Lemmas 3.2, (1)', 3.4, (1), 3.5, (1), 5.1 that $T u(y, z) \in G_{0, k_{0}}^{\{\sigma, \tau\}}(Y, Z)$ and that

$$
\begin{aligned}
\|T u\| \leq & \sum_{j=1}^{n-1} \frac{Y_{j+1}}{Y_{j}}\|u\| \\
& +\sum_{j=1}^{n} \frac{1}{Y_{j}}\left(\sum_{|\beta|+|\gamma|+r \geq 2}^{\text {finite }}\left|d_{j \beta \gamma r}\right|(Y, Z, \mathrm{~S}\|u\|) \mathcal{C}^{r-|\beta|-|\gamma|} Y^{\beta} Z^{\gamma}\|u\|^{r+1}\right) \\
& +\sum_{q=1}^{p} \frac{1}{Z_{q}}\left(\sum_{|\beta|+|\gamma|+r \geq 2}^{\text {finite }}\left|e_{q \beta \gamma r}\right|(Y, Z, \mathrm{~S}\|u\|) \mathcal{C}^{r-|\beta|-|\gamma|} Y^{\beta} Z^{\gamma}\|u\|^{r+1}\right) \\
& +\left|g_{0}\right|(Y, Z)+\frac{1}{\mathrm{~S}}|g|(Y, Z, \mathrm{~S}\|u\|) \\
\equiv & A(Y, Z,\|u\|) .
\end{aligned}
$$

Here for simplicity we write $\|\cdot\|_{k_{0}, Y, Z}^{\{\sigma, \tau\}}$ as $\|\cdot\|$. By the expression of $A(Y, Z,\|u\|)$ we can take $Y, Z$ and $\varepsilon$ such that $A(Y, Z, \varepsilon) \leq \varepsilon$. This shows that the operator $T: G_{0, k_{0}}^{\{\sigma, \tau\}}(Y, Z ; \varepsilon) \rightarrow G_{0, k_{0}}^{\{\sigma, \tau\}}(Y, Z ; \varepsilon)$ is well-defined.

Furthermore it follows from Lemmas 3.6, (1) and 5.2 that

$$
\begin{aligned}
\| T u & -T v \| \\
\leq & \|u-v\| \\
& \times\left[\sum_{j=1}^{n-1} \frac{Y_{j+1}}{Y_{j}}\right. \\
& +\mathrm{S} \sum_{j=1}^{n} \frac{1}{Y_{j}}\left(\sum_{|\beta|+|\gamma|+r \geq 2}^{\text {finite }}\left|d_{j \beta \gamma r, u}\right|(Y, Z, \mathrm{~S}(\|u\|+\|v\|)) \mathcal{C}^{r-|\beta|-|\gamma|} Y^{\beta} Z^{\gamma}\|u\|^{r+1}\right) \\
& +\sum_{j=1}^{n} \frac{1}{Y_{j}}\left(\sum_{|\beta|+|\gamma|+r \geq 2}^{\text {finite }}\left|d_{j \beta \gamma r}\right|(Y, Z, \mathrm{~S}\|v\|) \mathcal{C}^{r-|\beta|-|\gamma|} Y^{\beta} Z^{\gamma} \cdot \sum_{\nu=0}^{r}\|u\|^{r-\nu}\|v\|^{\nu}\right) \\
& +\mathrm{S} \sum_{q=1}^{p} \frac{1}{Z_{q}}\left(\sum_{|\beta|+|\gamma|+r \geq 2}^{\text {finite }}\left|e_{q \beta \gamma r, u}\right|(Y, Z, \mathrm{~S}(\|u\|+\|v\|)) \mathcal{C}^{r-|\beta|-|\gamma|} Y^{\beta} Z^{\gamma}\|u\|^{r+1}\right) \\
& +\sum_{q=1}^{p} \frac{1}{Z_{q}}\left(\sum_{|\beta|+|\gamma|+r \geq 2}^{\text {finite }}\left|e_{q \beta \gamma r}\right|(Y, Z, \mathrm{~S}\|v\|) \mathcal{C}^{r-|\beta|-|\gamma|} Y^{\beta} Z^{\gamma} \cdot \sum_{\nu=0}^{r}\|u\|^{r-\nu}\|v\|^{\nu}\right)
\end{aligned}
$$




$$
\begin{aligned}
& \left.+\left|g_{u}\right|(Y, Z, \mathrm{~S}(\|u\|+\|v\|))\right] \\
& \equiv\|u-v\| \times \widetilde{A}(Y, Z,\|u\|,\|v\|) .
\end{aligned}
$$

By the expression of $\widetilde{A}(Y, Z,\|u\|,\|v\|)$ we can take $Y, Z$ and $\varepsilon$ such that $\widetilde{A}(Y, Z, \varepsilon, \varepsilon)<1$. This shows that $T: G_{0, k_{0}}^{\{\sigma, \tau\}}(Y, Z ; \varepsilon) \rightarrow G_{0, k_{0}}^{\{\sigma, \tau\}}(Y, Z ; \varepsilon)$ is a contraction mapping. Therefore the proof is completed.

\section{§6. Proof of Theorem 2.1 (Cases (i), (ii), (iii))}

In this section we study Cases (i), (ii) and (iii). We only consider Case (i), and by the same reason as in the previous section we consider the case $k=1$. We write a formal power series solution as $u(x, y, z)=$ $\sum_{(\alpha, \beta, \gamma) \in \mathbf{N}^{m+n+p},|\alpha|+|\beta|+|\gamma| \geq 1} u_{\alpha \beta \gamma} x^{\alpha} y^{\beta} z^{\gamma} \quad(m+n+p=d)$ and use the Banach space $\widetilde{G}_{k, 0}^{\{\rho,(\sigma, \tau)\}}(X,(Y, Z))\left(\right.$ resp. $\left.G_{0, k}^{\{\rho, \sigma, \tau\}}(X, Y, Z)\right)\left(X=\left(X_{1}, \ldots, X_{m}\right)\right.$ $\in\left(\mathbf{R}_{+} \backslash\{0\}\right)^{m}, Y=\left(Y_{1}, \ldots, Y_{n}\right) \in\left(\mathbf{R}_{+} \backslash\{0\}\right)^{n}, Z=\left(Z_{1}, \ldots, Z_{p}\right) \in\left(\mathbf{R}_{+} \backslash\right.$ $\left.\{0\})^{p}\right)$. Therefore $u(x, y, z) \in \widetilde{G}_{k, 0}^{\{\rho,(\sigma, \tau)\}}(X,(Y, Z))\left(\right.$ resp. $\left.\in G_{0, k}^{\{\rho, \sigma, \tau\}}(X, Y, Z)\right)$ means

$$
\begin{aligned}
& \|\| u \|_{\substack{\{\rho, X,(Y, \tau)\} \\
k, Z)}} \\
& \quad \equiv \sum_{|\alpha|+|\beta|+|\gamma| \geq 1}\left|u_{\alpha \beta \gamma}\right| \frac{|\alpha| !(|\beta|+|\gamma|) !}{(\rho \cdot \alpha+\sigma \cdot \beta+\tau \cdot \gamma-k) !} X^{\alpha} Y^{\beta} Z^{\gamma}<+\infty \\
& \left(\text { resp. }\|u\|_{k, X, Y, Z}^{\{\rho, \sigma, \tau\}}\right. \\
& \left.\equiv \sum_{|\alpha|+|\beta|+|\gamma| \geq 1}\left|u_{\alpha \beta \gamma}\right| \frac{(|\alpha|+|\beta|+|\gamma|) !}{(\rho \cdot \alpha+\sigma \cdot \beta+\tau \cdot \gamma-k) !} X^{\alpha} Y^{\beta} Z^{\gamma}<+\infty\right) .
\end{aligned}
$$

We recall that the equation (2.1) is written as follows when $k=1$ :

$$
\begin{aligned}
\Lambda u(x, y, z)= & \left(P^{\prime}+P^{\prime \prime}+P^{\prime \prime \prime}+P^{\prime \prime \prime \prime}\right) u(x, y, z)+g_{0}(x, y, z) \\
& +g(x, y, z, u(x, y, z)), \\
u(0,0,0)= & 0,
\end{aligned}
$$

where $g_{0}$ and $g$ are holomorphic at the origin which satisfy $g_{0}(0,0,0)=0$ and $g(x, y, z, 0) \equiv 0, g_{u}(0,0,0,0)=0$, respectively, and

$$
P^{\prime} u=-\sum_{i=1}^{m-1} \delta_{i} x_{i+1} \frac{\partial u}{\partial x_{i}}+\sum_{i=1}^{m}\left(\sum_{\substack{|\alpha|+|\beta|+|\gamma|+r \geq 2 \\|\alpha| \geq 1}}^{\text {finite }} c_{i \alpha \beta \gamma r}(x, y, z, u) x^{\alpha} y^{\beta} z^{\gamma} u^{r}\right) \frac{\partial u}{\partial x_{i}},
$$




$$
\begin{aligned}
P^{\prime \prime} u= & \sum_{j=1}^{n}\left(\sum_{\substack{|\alpha|+|\beta|+|\gamma|+r \geq 2 \\
|\alpha| \geq 1}}^{\text {finite }} d_{j \alpha \beta \gamma r}(x, y, z, u) x^{\alpha} y^{\beta} z^{\gamma} u^{r}\right) \frac{\partial u}{\partial y_{j}} \\
& +\sum_{q=1}^{p}\left(\sum_{\substack{|\alpha|+|\beta|+|\gamma|+r \geq 2 \\
|\alpha| \geq 1}}^{\text {finite }} e_{q \alpha \beta \gamma r}(x, y, z, u) x^{\alpha} y^{\beta} z^{\gamma} u^{r}\right) \frac{\partial u}{\partial z_{q}}, \\
P^{\prime \prime \prime} u= & -\sum_{j=1}^{n-1} y_{j+1} \frac{\partial u}{\partial y_{j}}+\sum_{j=1}^{n}\left(\sum_{|\beta|+|\gamma|+r \geq 2}^{\text {finite }} d_{j \beta \gamma r}(x, y, z, u) y^{\beta} z^{\gamma} u^{r}\right) \frac{\partial u}{\partial y_{j}} \\
& +\sum_{q=1}^{p}\left(\sum_{|\beta|+|\gamma|+r \geq 2}^{\text {finite }} e_{q \beta \gamma r}(x, y, z, u) y^{\beta} z^{\gamma} u^{r}\right) \frac{\partial u}{\partial z_{q}}, \\
P^{\prime \prime \prime \prime} u= & \sum_{i=1}^{m}\left(\sum_{|\beta|+|\gamma|+r \geq 2}^{\text {finite }} c_{i \beta \gamma r}(x, y, z, u) y^{\beta} z^{\gamma} u^{r}\right) \frac{\partial u}{\partial x_{i}} .
\end{aligned}
$$

Here all coefficients $c_{i \alpha \beta \gamma r}, c_{i \beta \gamma r}, d_{j \alpha \beta \gamma r}, d_{j \beta \gamma r}, e_{q \alpha \beta \gamma r}$ and $e_{q \beta \gamma r}$ are holomorphic at the origin, and none of them vanish at the origin unless they vanish identically.

We assume that $s=(\rho, \sigma, \tau)$ satisfies the condition in Theorem 2.1, and prove that the formal solution of (6.1) belongs to $G^{\{\rho, \sigma, \tau\}}$. We remark that we admit the unique existence of the formal solution.

Proof of Case (i) of Theorem 2.1. First we remark that the operator $\Lambda$ is well-defined as the mapping from $G^{\{\rho, \sigma, \tau\}}$ to itself. Moreover it follows from the condition (Po) that $\Lambda: G^{\{\rho, \sigma, \tau\}} \rightarrow G^{\{\rho, \sigma, \tau\}}$ is bijective and $\Lambda^{-1}$ is given by

$$
\Lambda^{-1}\left(\sum_{(\alpha, \beta, \gamma) \in \mathbf{N}^{m+n+p}} U_{\alpha \beta \gamma} x^{\alpha} y^{\beta} z^{\gamma}\right)=\sum_{(\alpha, \beta, \gamma) \in \mathbf{N}^{m+n+p}} \frac{U_{\alpha \beta \gamma}}{\lambda \cdot \alpha-f_{u}(0,0)} x^{\alpha} y^{\beta} z^{\gamma} .
$$

Now we introduce a new unknown function $U(x, y, z)$ by

$$
U(x, y, z)=\Lambda u(x, y, z), \text { that is, } u(x, y, z)=\Lambda^{-1} U(x, y, z) .
$$

Then the equation (6.1) is equivalent to the following one:

$$
U(x, y, z)=T U(x, y, z), \quad U(0,0,0)=0,
$$

where 


$$
\begin{aligned}
T U(x, y, z)= & \left(P^{\prime}+P^{\prime \prime}+P^{\prime \prime \prime}+P^{\prime \prime \prime \prime}\right) \Lambda^{-1} U(x, y, z) \\
& +g_{0}(x, y, z)+g\left(x, y, z, \Lambda^{-1} U(x, y, z)\right) .
\end{aligned}
$$

Let us write the $\varepsilon$-closed ball in $\widetilde{G}_{0, k}^{\{\rho,(\sigma, \tau)\}}(X,(Y, Z))$ and $G_{0, k}^{\{\rho, \sigma, \tau\}}(X, Y, Z)$ as

$$
\begin{aligned}
& \widetilde{G}_{0, k}^{\{\rho,(\sigma, \tau)\}}(X,(Y, Z) ; \varepsilon)=\{U(x, y, z) \\
& \left.\quad=\sum_{|\alpha|+|\beta|+|\gamma| \geq 1} U_{\alpha \beta \gamma} x^{\alpha} y^{\beta} z^{\gamma} \in \widetilde{G}_{0, k}^{\{\rho,(\sigma, \tau)\}}(X,(Y, Z)) ; \||| U||_{k, X,(Y, Z)}^{\{\rho,(\sigma, \tau)\}} \leq \varepsilon\right\}
\end{aligned}
$$

and

$$
\begin{aligned}
& G_{0, k}^{\{\rho, \sigma, \tau\}}(X, Y, Z ; \varepsilon)=\{U(x, y, z) \\
& \left.\quad=\sum_{|\alpha|+|\beta|+|\gamma| \geq 1} U_{\alpha \beta \gamma} x^{\alpha} y^{\beta} z^{\gamma} \in G_{0, k}^{\{\rho, \sigma, \tau\}}(X, Y, Z) ;\|U\|_{k, X, Y, Z}^{\{\rho, \sigma\}} \leq \varepsilon\right\},
\end{aligned}
$$

respectively.

We shall prove that $T$ is well-defined as a mapping from $G$ to itself by choosing $k, X, Y, Z$ and $\varepsilon$ suitably and that it becomes a contraction mapping there, where

$$
G= \begin{cases}\widetilde{G}_{0, k}^{\{\rho,(\sigma, \tau)\}}(X,(Y, Z) ; \varepsilon) & \left(\begin{array}{l}
\text { when } P^{\prime \prime \prime \prime}=0 \text { or " } P^{\prime \prime}, P^{\prime \prime \prime \prime} \neq 0 \text { and } \\
s=(\rho, \sigma, \tau) \in \widetilde{S}_{1} \cap S_{1} \cap \widetilde{S}_{1}^{\prime} \cap S_{1}^{\prime \prime} "
\end{array}\right), \\
G_{0, k}^{\{\rho, \sigma, \tau\}}(X, Y, Z ; \varepsilon) & \left(\begin{array}{l}
\text { when } P^{\prime \prime}=0 \text { or } " P^{\prime \prime}, P^{\prime \prime \prime \prime} \neq 0 \text { and } \\
s=(\rho, \sigma, \tau) \in \widetilde{S}_{1} \cap S_{1} \cap S_{1}^{\prime} \cap \widetilde{S}_{1}^{\prime \prime} "
\end{array}\right) .\end{cases}
$$

The condition (Po) implies that there exists a positive constant $C$ such that

$$
\frac{1}{\left|\lambda \cdot \alpha-f_{u}(0,0)\right|} \leq C, \quad \frac{|\alpha|}{\left|\lambda \cdot \alpha-f_{u}(0,0)\right|} \leq C \text { for all } \alpha \in \mathbf{N}^{m}
$$

By the first inequality the linear operator $\Lambda^{-1}: \widetilde{G}_{0, k}^{\{\rho,(\sigma, \tau)\}}(X,(Y, Z)) \rightarrow$ $\widetilde{G}_{0, k}^{\{\rho,(\sigma, \tau)\}}(X,(Y, Z))\left(\operatorname{resp} . G_{0, k}^{\{\rho, \sigma, \tau\}}(X, Y, Z) \rightarrow G_{0, k}^{\{\rho, \sigma, \tau\}}(X, Y, Z)\right)$ is bounded and we have 


$$
\begin{aligned}
& \left\|\left|\Lambda^{-1} U\right|\right\|_{k, X,(Y, Z)}^{\{\rho,(\sigma, \tau)\}} \leq C \mid\|U\|_{k, X,(Y, Z)}^{\{\rho,(\sigma, \tau)\}} \text { for } U(x, y, z) \in \widetilde{G}_{0, k}^{\{\rho,(\sigma, \tau)\}}(X,(Y, Z)) \\
& \text { (resp. } \left.\left\|\Lambda^{-1} U\right\|_{k, X, Y, Z}^{\{\rho, \sigma, \tau\}} \leq C\|U\|_{k, X, Y, Z}^{\{\rho, \sigma, \tau\}} \text { for } U(x, y, z) \in G_{0, k}^{\{\rho, \sigma, \tau\}}(X, Y, Z)\right) .
\end{aligned}
$$

Here let us give some lemmas.

Lemma 6.1. (1) Let $|\alpha|+|\beta|+|\gamma|+r \geq 1$ and $\left|\alpha^{\prime}\right|+\left|\beta^{\prime}\right|+\left|\gamma^{\prime}\right| \geq 1$. Let us assume that $(\rho, \sigma, \tau) \in[1,+\infty)^{d}$ satisfies

$$
\begin{gathered}
\rho \cdot\left(\alpha+r \mathrm{e}_{i}^{(m)}-\alpha^{\prime}\right)+\sigma \cdot\left(\beta-\beta^{\prime}\right)+\tau \cdot\left(\gamma-\gamma^{\prime}\right) \\
\geq|\alpha|+|\beta|+|\gamma|+r \quad(i=1, \ldots, m), \\
\rho \cdot\left(\alpha-\alpha^{\prime}\right)+\sigma \cdot\left(\beta+r \mathrm{e}_{j}^{(n)}-\beta^{\prime}\right)+\tau \cdot\left(\gamma-\gamma^{\prime}\right) \\
\geq|\alpha|+|\beta|+|\gamma|+r \quad(j=1, \ldots, n), \\
\rho \cdot\left(\alpha-\alpha^{\prime}\right)+\sigma \cdot\left(\beta-\beta^{\prime}\right)+\tau \cdot\left(\gamma+r \mathrm{e}_{q}^{(p)}-\gamma^{\prime}\right) \\
\geq|\alpha|+|\beta|+|\gamma|+r \quad(q=1, \ldots, p),
\end{gathered}
$$

where $\mathrm{e}_{i}^{(m)}=\left(\delta_{i 1}, \ldots, \delta_{i m}\right)(i=1, \ldots, m), \mathrm{e}_{j}^{(n)}=\left(\delta_{j 1}, \ldots, \delta_{j n}\right)(j=1, \ldots, n)$ and $\mathrm{e}_{q}^{(p)}=\left(\delta_{q 1}, \ldots, \delta_{q p}\right)(q=1, \ldots, p)$. If $k$ satisfies

$$
\begin{aligned}
& \frac{(1-\rho) \cdot \alpha+(1-\sigma) \cdot \beta+(1-\tau) \cdot \gamma+\rho \cdot \alpha^{\prime}+\sigma \cdot \beta^{\prime}+\tau \cdot \gamma^{\prime}}{r} \\
& \quad \leq k \leq \min \left\{\rho_{1}-1, \ldots, \rho_{m}-1, \sigma_{1}-1, \ldots, \sigma_{n}-1, \tau_{1}-1, \ldots, \tau_{p}-1\right\}
\end{aligned}
$$

and $U_{1}, \ldots, U_{r}, V \in \widetilde{G}_{0, k}^{\{\rho,(\sigma, \tau)\}}(X,(Y, Z))$ (resp. $\left.G_{0, k}^{\{\rho, \sigma, \tau\}}(X, Y, Z)\right)$, then it holds that $x^{\alpha} y^{\beta} z^{\gamma}\left\{\prod_{\mu=1}^{r}\left(\Lambda^{-1} U_{\mu}\right)\right\} D_{x}{ }^{\alpha^{\prime}} D_{y}{ }^{\beta^{\prime}} D_{z}{ }^{\gamma^{\prime}} \Lambda^{-1} V \in \widetilde{G}_{0, k}^{\{\rho,(\sigma, \tau)\}}(X,(Y, Z))$ (resp. $\left.G_{0, k}^{\{\rho, \sigma, \tau\}}(X, Y, Z)\right)$ and that

$$
\begin{gathered}
\left\|\left|x^{\alpha} y^{\beta} z^{\gamma}\left(\Lambda^{-1} U_{1}\right) \cdots\left(\Lambda^{-1} U_{r}\right) D_{x}^{\alpha^{\prime}} D_{y}^{\beta^{\prime}} D_{z}^{\gamma^{\prime}} \Lambda^{-1} V\right|\right\|_{k, X,(Y, Z)}^{\{\rho,(\sigma, \tau)\}} \\
\leq C^{r+1} \mathcal{C}^{r-|\alpha|-|\beta|-|\gamma|} \frac{X^{\alpha}}{X^{\alpha^{\prime}}} \frac{Y^{\beta}}{Y^{\beta^{\prime}}} \frac{Z^{\gamma}}{Z^{\gamma^{\prime}}} \\
\times\left\|| | U _ { 1 } \left|\left\|_ { k , X , ( Y , Z ) } ^ { \{ \rho , ( \sigma , \tau ) \} } \cdots \left|\left\|U _ { r } \left|\left\|_{k, X,(Y, Z)}^{\{\rho,(\sigma, \tau)\}} \mid\right\| V \|_{k, X,(Y, Z)}^{\{\rho,(\sigma, \tau)\}}\right.\right.\right.\right.\right.\right. \\
\left(\begin{array}{c}
\left\|x^{\alpha} y^{\beta} z^{\gamma}\left(\Lambda^{-1} U_{1}\right) \cdots\left(\Lambda^{-1} U_{r}\right) D_{x}^{\alpha^{\prime}} D_{y}^{\beta^{\prime}} D_{z}^{\gamma^{\prime}} \Lambda^{-1} V\right\|_{k, X, Y, Z}^{\{\rho, \sigma, \tau\}} \\
\leq e s p . \quad C^{r+1} \mathcal{C}^{r-|\alpha|-|\beta|-|\gamma|} \frac{X^{\alpha}}{X^{\alpha^{\prime}}} \frac{Y^{\beta}}{Y^{\beta^{\prime}}} \frac{Z^{\gamma}}{Z^{\gamma^{\prime}}} \\
\times\left\|U_{1}\right\|_{k, X, Y, Z}^{\{\rho, \sigma, \tau\}} \cdots\left\|U_{r}\right\|_{k, X, Y, Z}^{\{\rho, \sigma, \tau\}}\|V\|_{k, X, Y, Z}^{\{\rho, \sigma, \tau\}}
\end{array}\right),
\end{gathered}
$$

where $C$ is the same constant as in (6.4), and 


$$
\mathcal{C}= \begin{cases}1 & (\text { when }|\alpha|+|\beta|+|\gamma| \geq r), \\ \max \left\{\rho_{1}, \ldots, \rho_{m}, \sigma_{1}, \ldots, \sigma_{n}, \tau_{1}, \ldots, \tau_{p}\right\} & (\text { when }|\alpha|+|\beta|+|\gamma| \leq r-1) .\end{cases}
$$

(2) Let $|\alpha|+|\beta|+|\gamma|+r \geq 1$ and $\left|\alpha^{\prime}\right| \geq 1$. Let us assume that $(\rho, \sigma, \tau)$ $\in[1,+\infty)^{d}$ satisfies

$$
\begin{aligned}
& \rho \cdot\left(\alpha+r \mathrm{e}_{i}^{(m)}-\alpha^{\prime}\right)+\sigma \cdot\left(\beta-\beta^{\prime}\right)+\tau \cdot\left(\gamma-\gamma^{\prime}\right) \geq|\alpha|+|\beta|+|\gamma|+r-1, \\
& \rho \cdot\left(\alpha-\alpha^{\prime}\right)+\sigma \cdot\left(\beta+r \mathrm{e}_{j}^{(n)}-\beta^{\prime}\right)+\tau \cdot\left(\gamma-\gamma^{\prime}\right) \geq|\alpha|+|\beta|+|\gamma|+r-1 \\
& \rho \cdot\left(\alpha-\alpha^{\prime}\right)+\sigma \cdot\left(\beta-\beta^{\prime}\right)+\tau \cdot\left(\gamma+r \mathrm{e}_{q}^{(p)}-\gamma^{\prime}\right) \geq|\alpha|+|\beta|+|\gamma|+r-1
\end{aligned}
$$

$(i=1, \ldots, m ; j=1, \ldots, n ; q=1, \ldots, p)$. If $k$ satisfies

$$
\begin{aligned}
& \frac{(1-\rho) \cdot \alpha+(1-\sigma) \cdot \beta+(1-\tau) \cdot \gamma+\rho \cdot \alpha^{\prime}+\sigma \cdot \beta^{\prime}+\tau \cdot \gamma^{\prime}-1}{r} \\
& \quad \leq k \leq \min \left\{\rho_{1}-1, \ldots, \rho_{m}-1, \sigma_{1}-1, \ldots, \sigma_{n}-1, \tau_{1}-1, \ldots, \tau_{p}-1\right\},
\end{aligned}
$$

and $U_{1}, \ldots, U_{r}, \quad V \in G_{0, k}^{\{\rho, \sigma, \tau\}}(X, Y, Z)$, then $x^{\alpha} y^{\beta} z^{\gamma}\left\{\prod_{\mu=1}^{r}\left(\Lambda^{-1} U_{\mu}\right)\right\}$ $\times D_{x}{ }^{\alpha^{\prime}} D_{y}{ }^{\beta^{\prime}} D_{z}{ }^{\gamma^{\prime}} \Lambda^{-1} V \in G_{0, k}^{\{\rho, \sigma, \tau\}}(X, Y, Z)$ and

$$
\begin{aligned}
& \left\|x^{\alpha} y^{\beta} z^{\gamma}\left(\Lambda^{-1} U_{1}\right) \cdots\left(\Lambda^{-1} U_{r}\right) D_{x}{ }^{\alpha^{\prime}} D_{y}^{\beta^{\prime}} D_{z}^{\gamma^{\prime}} \Lambda^{-1} V\right\|_{k, X, Y, Z}^{\{\rho, \sigma\}} \\
& \quad \leq C^{r+1} \widetilde{\mathcal{C}}^{r-|\alpha|-|\beta|-|\gamma|+1} \frac{X^{\alpha}}{X^{\alpha^{\prime}}} \frac{Y^{\beta}}{Y^{\beta^{\prime}}} \frac{Z^{\gamma}}{Z^{\gamma^{\prime}}}\left\|U_{1}\right\|_{k, X, Y, Z}^{\{\rho, \sigma, \tau\}} \cdots\left\|U_{r}\right\|_{k, X, Y, Z}^{\{\rho, \sigma, \tau\}}\|V\|_{k, X, Y, Z}^{\{\rho, \sigma, \tau\}},
\end{aligned}
$$

where

$$
\widetilde{\mathcal{C}}= \begin{cases}1 & (\text { when }|\alpha|+|\beta|+|\gamma| \geq r+1), \\ \max \left\{\rho_{1}, \ldots, \rho_{m}, \sigma_{1}, \ldots, \sigma_{n}, \tau_{1}, \ldots, \tau_{p}\right\} & (\text { when }|\alpha|+|\beta|+|\gamma| \leq r) .\end{cases}
$$

(3) Let $|\alpha| \geq 1$ and $\left|\alpha^{\prime}\right|+\left|\beta^{\prime}\right|+\left|\gamma^{\prime}\right| \geq 1$. Let us assume that $(\rho, \sigma, \tau) \in$ $[1,+\infty)^{d}$ satisfies (6.10). If $k$ satisfies $(6.11)$, and $U_{1}, \ldots, U_{r}, V \in \widetilde{G}_{0, k}^{\{\rho,(\sigma, \tau)\}}(X$, $(Y, Z))$, then $x^{\alpha} y^{\beta} z^{\gamma}\left\{\prod_{\mu=1}^{r}\left(\Lambda^{-1} U_{\mu}\right)\right\} D_{x}{ }^{\alpha^{\prime}} D_{y}{ }^{\beta^{\prime}} D_{z}{ }^{\gamma^{\prime}} \Lambda^{-1} V \in \widetilde{G}_{0, k}^{\{\rho,(\sigma, \tau)\}}(X$, $(Y, Z))$ and

$$
\begin{aligned}
\left\|\left|x^{\alpha} y^{\beta} z^{\gamma}\left(\Lambda^{-1} U_{1}\right) \cdots\left(\Lambda^{-1} U_{r}\right) D_{x}{ }^{\alpha^{\prime}} D_{y}{ }^{\beta^{\prime}} D_{z} \gamma^{\prime} \Lambda^{-1} V\right|\right\|_{k, X,(Y, Z)}^{\{\rho,(\sigma, \tau)\}} \\
\leq C_{r \alpha \alpha^{\prime}} \widetilde{\mathcal{C}}^{r-|\alpha|-|\beta|-|\gamma|+1} \frac{X^{\alpha}}{X^{\alpha^{\prime}}} \frac{Y^{\beta}}{Y^{\beta^{\prime}}} \frac{Z^{\gamma}}{Z^{\gamma^{\prime}}} \\
\quad \times\left\|\left|U_{1}\right|\right\|_{k, X,(Y, Z)}^{\{\rho,(\sigma, \tau)\}} \cdots\left|\left\|U_{r}\right\|\right|_{k, X,(Y, Z)}^{\{\rho,(\sigma, \tau)\}} \mid\|V\|_{k, X,(Y, Z)}^{\{\rho,(\sigma, \tau)\}},
\end{aligned}
$$


where $C_{r \alpha \alpha^{\prime}}$ is the constant dependent of $r, \alpha$ and $\alpha^{\prime}$.

Remark 6.1. The conditions (6.6) and (6.10) imply

$$
\begin{aligned}
& \frac{(1-\rho) \cdot \alpha+(1-\sigma) \cdot \beta+(1-\tau) \cdot \gamma+\rho \cdot \alpha^{\prime}+\sigma \cdot \beta^{\prime}+\tau \cdot \gamma^{\prime}}{r} \\
& \quad \leq \min \left\{\rho_{1}-1, \ldots, \rho_{m}-1, \sigma_{1}-1, \ldots, \sigma_{n}-1, \tau_{1}-1, \ldots, \tau_{p}-1\right\}
\end{aligned}
$$

and

$$
\begin{aligned}
& \frac{(1-\rho) \cdot \alpha+(1-\sigma) \cdot \beta+(1-\tau) \cdot \gamma+\rho \cdot \alpha^{\prime}+\sigma \cdot \beta^{\prime}+\tau \cdot \gamma^{\prime}-1}{r} \\
& \quad \leq \min \left\{\rho_{1}-1, \ldots, \rho_{m}-1, \sigma_{1}-1, \ldots, \sigma_{n}-1, \tau_{1}-1, \ldots, \tau_{p}-1\right\}
\end{aligned}
$$

respectively.

Remark 6.2. Let us write the Newton polyhedron of $x^{\alpha} y^{\beta} z^{\gamma} u^{r}$ $\times D_{x}{ }^{\alpha^{\prime}} D_{y}{ }^{\beta^{\prime}} D_{z}{ }^{\gamma^{\prime}} u$ as

$$
\begin{aligned}
N & \left(x^{\alpha} y^{\beta} z^{\gamma} u^{r} D_{x}{ }^{\alpha^{\prime}} D_{y}{ }^{\beta^{\prime}} D_{z} \gamma^{\prime} u\right) \\
= & \operatorname{Ch}\left\{\bigcup_{i=1}^{m} Q\left(\alpha+r \mathrm{e}_{i}^{(m)}, \beta, \gamma, \alpha^{\prime}, \beta^{\prime}, \gamma^{\prime}\right) \cup \bigcup_{j=1}^{n} Q\left(\alpha, \beta+r \mathrm{e}_{j}^{(n)}, \gamma, \alpha^{\prime}, \beta^{\prime}, \gamma^{\prime}\right)\right. \\
& \left.\cup \bigcup_{q=1}^{p} Q\left(\alpha, \beta, \gamma+r \mathrm{e}_{q}^{(p)}, \alpha^{\prime}, \beta^{\prime}, \gamma^{\prime}\right)\right\}
\end{aligned}
$$

where

$$
\begin{aligned}
& Q\left(\alpha, \beta, \gamma, \alpha^{\prime}, \beta^{\prime}, \gamma^{\prime}\right)
\end{aligned}
$$

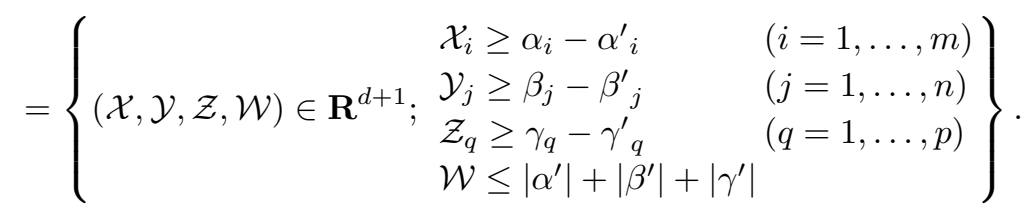

Furthermore we define $\Pi(\rho, \sigma, \tau)$ and $\widetilde{\Pi}(\rho, \sigma, \tau)$ by

$$
\begin{aligned}
\Pi(\rho, \sigma, \tau)=\left\{(\mathcal{X}, \mathcal{Y}, \mathcal{Z}, \mathcal{W}) \in \mathbf{R}^{d+1} ;\right. & \left(\rho-1^{(m)}\right) \cdot \mathcal{X} \\
& \left.+\left(\sigma-1^{(n)}\right) \cdot \mathcal{Y}+\left(\tau-1^{(p)}\right) \cdot \mathcal{Z}-\mathcal{W} \geq 0\right\}
\end{aligned}
$$

and 


$$
\begin{aligned}
\widetilde{\Pi}(\rho, \sigma, \tau)=\left\{(\mathcal{X}, \mathcal{Y}, \mathcal{Z}, \mathcal{W}) \in \mathbf{R}^{d+1}\right. & ;\left(\rho-1^{(m)}\right) \cdot \mathcal{X} \\
& \left.+\left(\sigma-1^{(n)}\right) \cdot \mathcal{Y}+\left(\tau-1^{(p)}\right) \cdot \mathcal{Z}-\mathcal{W} \geq-1\right\},
\end{aligned}
$$

respectively, and define $S$ and $\widetilde{S}$ as follows:

$$
\begin{aligned}
& S=\left\{(\rho, \sigma, \tau) \in[1,+\infty)^{d} ; N\left(x^{\alpha} y^{\beta} z^{\gamma} u^{r} D_{x}^{\alpha^{\prime}} D_{y}^{\beta^{\prime}} D_{z}^{\gamma^{\prime}} u\right) \subset \Pi(\rho, \sigma, \tau)\right\}, \\
& \widetilde{S}=\left\{(\rho, \sigma, \tau) \in[1,+\infty)^{d} ; N\left(x^{\alpha} y^{\beta} z^{\gamma} u^{r} D_{x}^{\alpha^{\prime}} D_{y}^{\beta^{\prime}} D_{z}^{\gamma^{\prime}} u\right) \subset \widetilde{\Pi}(\rho, \sigma, \tau)\right\} .
\end{aligned}
$$

Then the conditions $(\rho, \sigma, \tau) \in S$ and $(\rho, \sigma, \tau) \in \widetilde{S}$ are equivalent to (6.6) and (6.10), respectively.

Proof of Lemma 6.1. For $U_{\mu}(x, y, z)=\sum_{\left|\alpha^{\mu}\right|+\left|\beta^{\mu}\right|+\left|\gamma^{\mu}\right| \geq 1} U_{\alpha^{\mu} \beta^{\mu} \gamma^{\mu}}$ $\times x^{\alpha^{\mu}} y^{\beta^{\mu}} z^{\gamma^{\mu}}(\mu=1,2, \ldots, r)$ and $V(x, y, z)=\sum_{|\widetilde{\alpha}|+|\widetilde{\beta}|+|\widetilde{\gamma}| \geq 1} V_{\widetilde{\alpha} \widetilde{\beta} \widetilde{\gamma}} x^{\widetilde{\alpha}} y^{\widetilde{\beta}} z^{\widetilde{\gamma}}$ it holds that

$$
\begin{aligned}
& x^{\alpha} y^{\beta} z^{\gamma}\left(\Lambda^{-1} U_{1}\right) \cdots\left(\Lambda^{-1} U_{r}\right) D_{x}{ }^{\alpha^{\prime}} D_{y}^{\beta^{\prime}} D_{z^{\gamma^{\prime}}} \Lambda^{-1} V(x, y, z) \\
& =\sum_{\substack{\left|\alpha^{1}\right|+\left|\beta^{1}\right|+\left|1^{1}\right| \geq 1, \ldots,\left|\alpha^{r}\right|+\left|\beta^{r}\right|+\left|\gamma^{r}\right| \geq 1 \\
\widetilde{\alpha} \geq \alpha^{\prime}, \widetilde{\beta} \geq \beta^{\prime}, \tilde{\gamma} \geq \gamma^{\prime}}}\left\{\prod_{\mu=1}^{r} \frac{1}{\lambda \cdot \alpha^{\mu}-f_{u}(0,0)}\right\} \frac{1}{\lambda \cdot \widetilde{\alpha}-f_{u}(0,0)} \\
& \quad \times \frac{\widetilde{\alpha} !}{\left(\widetilde{\alpha}-\alpha^{\prime}\right) !} \frac{\widetilde{\beta} !}{\left(\widetilde{\beta}-\beta^{\prime}\right) !} \frac{\widetilde{\gamma} !}{\left(\widetilde{\gamma}-\gamma^{\prime}\right) !}\left\{\prod_{\mu=1}^{r} U_{\alpha^{\mu} \beta^{\mu} \gamma^{\mu}}^{\mu}\right\} \\
& \quad \times V_{\widetilde{\alpha} \widetilde{\beta} \widetilde{\gamma}} x^{\alpha+\bar{\alpha}+\widetilde{\alpha}-\alpha^{\prime}} y^{\beta+\bar{\beta}+\widetilde{\beta}-\beta^{\prime}} z^{\gamma+\bar{\gamma}+\widetilde{\gamma}-\gamma^{\prime}},
\end{aligned}
$$

where $\bar{\alpha}=\alpha^{1}+\cdots+\alpha^{r}, \bar{\beta}=\beta^{1}+\cdots+\beta^{r}, \bar{\gamma}=\gamma^{1}+\cdots+\gamma^{r}$, which implies that

$$
\begin{aligned}
& \|\left.|| x^{\alpha} y^{\beta} z^{\gamma}\left(\Lambda^{-1} U_{1}\right) \cdots\left(\Lambda^{-1} U_{r}\right) D_{x}^{\alpha^{\prime}} D_{y}^{\beta^{\prime}} D_{z}^{\gamma^{\prime}} \Lambda^{-1} V||\right|_{k, X,(Y, Z)} ^{\{\rho,(\sigma, \tau)\}} \\
& =\sum_{\substack{\left|\alpha^{1}\right|+\left|\beta^{1}\right|+\left|\gamma^{1}\right| \geq 1 \\
\vdots \\
\left|\alpha^{r}\right|+\left|\beta^{r}\right|+\left|\gamma^{r}\right| \geq 1 \\
\widetilde{\alpha} \geq \alpha^{\prime}, \widetilde{\beta} \geq \beta^{\prime}, \widetilde{\gamma} \geq \gamma^{\prime}}}\left\{\prod_{\mu=1}^{r} \frac{1}{\left|\lambda \cdot \alpha^{\mu}-f_{u}(0,0)\right|}\right\} \frac{1}{\left|\lambda \cdot \widetilde{\alpha}-f_{u}(0,0)\right|} \\
& \quad \times \frac{\widetilde{\alpha} !}{\left(\widetilde{\alpha}-\alpha^{\prime}\right) !} \frac{\widetilde{\beta} !}{\left(\widetilde{\beta}-\beta^{\prime}\right) !} \frac{\widetilde{\gamma} !}{\left(\widetilde{\gamma}-\gamma^{\prime}\right) !} \\
& \quad \times \frac{\left|\alpha+\bar{\alpha}+\widetilde{\alpha}-\alpha^{\prime}\right| !\left(\left|\beta+\bar{\beta}+\widetilde{\beta}-\beta^{\prime}\right|+\left|\gamma+\bar{\gamma}+\widetilde{\gamma}-\gamma^{\prime}\right|\right) !}{\left\{\rho \cdot\left(\alpha+\bar{\alpha}+\widetilde{\alpha}-\alpha^{\prime}\right)+\sigma \cdot\left(\beta+\bar{\beta}+\widetilde{\beta}-\beta^{\prime}\right)+\tau \cdot\left(\gamma+\bar{\gamma}+\widetilde{\gamma}-\gamma^{\prime}\right)-k\right\} !}
\end{aligned}
$$




$$
\times\left\{\prod_{\mu=1}^{r}\left|U_{\alpha^{\mu} \beta^{\mu} \gamma^{\mu}}^{\mu}\right|\right\}\left|V_{\widetilde{\alpha} \widetilde{\gamma}}\right| X^{\alpha+\bar{\alpha}+\widetilde{\alpha}-\alpha^{\prime}} Y^{\beta+\bar{\beta}+\widetilde{\beta}-\beta^{\prime}} Z^{\gamma+\bar{\gamma}+\widetilde{\gamma}-\gamma^{\prime}}
$$

and

$$
\begin{aligned}
& \left\|x^{\alpha} y^{\beta} z^{\gamma}\left(\Lambda^{-1} U_{1}\right) \cdots\left(\Lambda^{-1} U_{r}\right) D_{x}{ }^{\alpha^{\prime}} D_{y}^{\beta^{\prime}} D_{z}{ }^{\gamma^{\prime}} \Lambda^{-1} V\right\|_{k, X, Y, Z}^{\{\rho, \sigma, \tau\}} \\
& =\sum_{\left|\alpha^{1}\right|+\left|\beta^{1}\right|+\left|\gamma^{1}\right| \geq 1}\left\{\prod_{\mu=1}^{r} \frac{1}{\left|\lambda \cdot \alpha^{\mu}-f_{u}(0,0)\right|}\right\} \frac{1}{\left|\lambda \cdot \widetilde{\alpha}-f_{u}(0,0)\right|} \\
& \left|\alpha^{r}\right|+\left|\beta^{r} \dot{r}\right|+\left|\gamma^{r}\right| \geq 1 \\
& \times \frac{\widetilde{\alpha} !}{\left(\widetilde{\alpha}-\alpha^{\prime}\right) !} \frac{\widetilde{\beta} !}{\left(\widetilde{\beta}-\beta^{\prime}\right) !} \frac{\widetilde{\gamma} !}{\left(\widetilde{\gamma}-\gamma^{\prime}\right) !} \\
& \times \frac{\left(\left|\alpha+\bar{\alpha}+\widetilde{\alpha}-\alpha^{\prime}\right|+\left|\beta+\bar{\beta}+\widetilde{\beta}-\beta^{\prime}\right|+\left|\gamma+\bar{\gamma}+\widetilde{\gamma}-\gamma^{\prime}\right|\right) !}{\left\{\rho \cdot\left(\alpha+\bar{\alpha}+\widetilde{\alpha}-\alpha^{\prime}\right)+\sigma \cdot\left(\beta+\bar{\beta}+\widetilde{\beta}-\beta^{\prime}\right)+\tau \cdot\left(\gamma+\bar{\gamma}+\widetilde{\gamma}-\gamma^{\prime}\right)-k\right\} !} \\
& \times\left\{\prod_{\mu=1}^{r}\left|U_{\alpha^{\mu} \beta^{\mu} \gamma^{\mu}}^{\mu}\right|\right\}\left|V_{\widetilde{\alpha} \widetilde{\beta}}\right| X^{\alpha+\bar{\alpha}+\widetilde{\alpha}-\alpha^{\prime}} Y^{\beta+\bar{\beta}+\widetilde{\beta}-\beta^{\prime}} Z^{\gamma+\bar{\gamma}+\widetilde{\gamma}-\gamma^{\prime}} .
\end{aligned}
$$

(1): The case $U_{1}, \ldots, U_{r}, V \in G_{0, k}^{\{\rho, \sigma, \tau\}}(X, Y, Z)$ : By using the first inequality of (6.4) we can prove the lemma similarly to the proof of Lemma 5.1.

The case $U_{1}, \ldots, U_{r}, V \in \widetilde{G}_{0, k}^{\{\rho,(\sigma, \tau)\}}(X,(Y, Z))$ : From the first inequality of (6.4) it is sufficient to prove the following inequality:

$$
\begin{aligned}
& \frac{\widetilde{\alpha} !}{\left(\widetilde{\alpha}-\alpha^{\prime}\right) !} \frac{\widetilde{\beta} !}{\left(\widetilde{\beta}-\beta^{\prime}\right) !} \frac{\widetilde{\gamma} !}{\left(\widetilde{\gamma}-\gamma^{\prime}\right) !} \\
& \times \frac{\left\{\prod_{\mu=1}^{r}\left(\rho \cdot \alpha^{\mu}+\sigma \cdot \beta^{\mu}+\tau \cdot \gamma^{\mu}-k\right) !\right\}(\rho \cdot \widetilde{\alpha}+\sigma \cdot \widetilde{\beta}+\tau \cdot \widetilde{\gamma}-k) !}{\left\{\rho \cdot\left(\alpha+\bar{\alpha}+\widetilde{\alpha}-\alpha^{\prime}\right)+\sigma \cdot\left(\beta+\bar{\beta}+\widetilde{\beta}-\beta^{\prime}\right)+\tau \cdot\left(\gamma+\bar{\gamma}+\widetilde{\gamma}-\gamma^{\prime}\right)-k\right\} !} \\
& \leq \mathcal{C}^{r-|\alpha|-|\beta|-|\gamma|} \frac{\left\{\prod_{\mu=1}^{r}\left|\alpha^{\mu}\right| !\left(\left|\beta^{\mu}\right|+\left|\gamma^{\mu}\right|\right) !\right\}|\widetilde{\alpha}| !(|\widetilde{\beta}|+|\widetilde{\gamma}|) !}{\left|\alpha+\bar{\alpha}+\widetilde{\alpha}-\alpha^{\prime}\right| !\left(\left|\beta+\bar{\beta}+\widetilde{\beta}-\beta^{\prime}\right|+\left|\gamma+\bar{\gamma}+\widetilde{\gamma}-\gamma^{\prime}\right|\right) !} \\
& \quad\left(\left|\alpha^{1}\right|+\left|\beta^{1}\right|+\left|\gamma^{1}\right| \geq 1, \ldots,\left|\alpha^{r}\right|+\left|\beta^{r}\right|+\left|\gamma^{r}\right| \geq 1,\right. \\
& \left.\widetilde{\alpha} \geq \alpha^{\prime}, \widetilde{\beta} \geq \beta^{\prime}, \widetilde{\gamma} \geq \gamma^{\prime}\right) .
\end{aligned}
$$

If we admit the following inequality (6.16), the proof of (6.15) is similar to 
the proof of (5.6).

$$
\begin{aligned}
& \frac{\left\{\prod_{\mu=1}^{r}\left(\left|\alpha^{\mu}\right|+\left|\beta^{\mu}\right|+\left|\gamma^{\mu}\right|\right) !\right\}(|\widetilde{\alpha}|+|\widetilde{\beta}|+|\widetilde{\gamma}|) !}{(|\bar{\alpha}+\widetilde{\alpha}|+|\bar{\beta}+\widetilde{\beta}|+|\bar{\gamma}+\widetilde{\gamma}|) !} \\
& \quad \leq \frac{\left\{\prod_{\mu=1}^{r}\left|\alpha^{\mu}\right| !\left(\left|\beta^{\mu}\right|+\left|\gamma^{\mu}\right|\right) !\right\}|\widetilde{\alpha}| !(|\widetilde{\beta}|+|\widetilde{\gamma}|) !}{|\bar{\alpha}+\widetilde{\alpha}| !(|\bar{\beta}+\widetilde{\beta}|+|\bar{\gamma}+\widetilde{\gamma}|) !} .
\end{aligned}
$$

The proof of (6.16) is reduced to that of the following inequality: For $a_{1}, \ldots, a_{r}$, $b_{1}, \ldots, b_{r} \geq 0$,

$$
\frac{\left(a_{1}+b_{1}\right) ! \cdots\left(a_{r}+b_{r}\right) !}{\left(a_{1}+\cdots+a_{r}+b_{1}+\cdots+b_{r}\right) !} \leq \frac{a_{1} ! b_{1} ! \cdots a_{r} ! b_{r} !}{\left(a_{1}+\cdots+a_{r}\right) !\left(b_{1}+\cdots+b_{r}\right) !} .
$$

(6.17) is proved as follows: Let us consider the equality

$$
(\xi+\eta)^{a_{1}+b_{1}} \cdots(\xi+\eta)^{a_{r}+b_{r}}=(\xi+\eta)^{a_{1}+\cdots+a_{r}+b_{1}+\cdots+b_{r}},
$$

and let us calculate the coefficients of $\xi^{a_{1}+\cdots+a_{r}} \eta^{b_{1}+\cdots+b_{r}}$ in both sides. Then we have

$$
\begin{aligned}
\sum_{\substack{1 \leq c_{1} \leq a_{1}+b_{1}, \ldots, 1 \leq c_{r} \leq a_{r}+b_{r} \\
c_{1}+\cdots+c_{r}=a_{1}+\cdots+a_{r}}}\left(\begin{array}{c}
a_{1}+b_{1} \\
c_{1}
\end{array}\right) & \cdots\left(\begin{array}{c}
a_{r}+b_{r} \\
c_{r}
\end{array}\right) \\
= & \left(\begin{array}{c}
a_{1}+\cdots+a_{r}+b_{1}+\cdots+b_{r} \\
a_{1}+\cdots+a_{r}
\end{array}\right),
\end{aligned}
$$

which implies

$$
\begin{aligned}
& \frac{\left(a_{1}+b_{1}\right) !}{a_{1} ! b_{1} !} \cdots \frac{\left(a_{r}+b_{r}\right) !}{a_{r} ! b_{r} !} \\
& \quad=\left(\begin{array}{c}
a_{1}+b_{1} \\
a_{1}
\end{array}\right) \cdots\left(\begin{array}{c}
a_{r}+b_{r} \\
a_{r}
\end{array}\right) \leq\left(\begin{array}{c}
a_{1}+\cdots+a_{r}+b_{1}+\cdots+b_{r} \\
a_{1}+\cdots+a_{r}
\end{array}\right) \\
& \quad=\frac{\left(a_{1}+\cdots+a_{r}+b_{1}+\cdots+b_{r}\right) !}{\left(a_{1}+\cdots+a_{r}\right) !\left(b_{1}+\cdots+b_{r}\right) !} .
\end{aligned}
$$

Therefore (6.17) is proved.

(2): Let us estimate as $\left|1 /\left(\lambda \cdot \alpha^{\mu}-f_{u}(0,0)\right)\right| \leq C(\mu=1, \ldots, r),|\widetilde{\alpha}| /(\lambda$. $\left.\widetilde{\alpha}-f_{u}(0,0)\right) \mid \leq C$, and note that

$$
\frac{\widetilde{\alpha} !}{\left(\widetilde{\alpha}-\alpha^{\prime}\right) !} \frac{\widetilde{\beta} !}{\left(\widetilde{\beta}-\beta^{\prime}\right) !} \frac{\widetilde{\gamma} !}{\left(\widetilde{\gamma}-\gamma^{\prime}\right) !} \leq|\widetilde{\alpha}| \frac{(|\widetilde{\alpha}|+|\widetilde{\beta}|+|\widetilde{\gamma}|-1) !}{\left(|\widetilde{\alpha}|+|\widetilde{\beta}|+|\widetilde{\gamma}|-\left|\alpha^{\prime}\right|-\left|\beta^{\prime}\right|-\left|\gamma^{\prime}\right|\right) !}
$$

Then it is sufficient to prove the following inequality:

$$
\frac{(|\widetilde{\alpha}|+|\widetilde{\beta}|+|\widetilde{\gamma}|-1) !}{\left(|\widetilde{\alpha}|+|\widetilde{\beta}|+|\widetilde{\gamma}|-\left|\alpha^{\prime}\right|-\left|\beta^{\prime}\right|-\left|\gamma^{\prime}\right|\right) !}
$$




$$
\begin{aligned}
& \times \frac{\left\{\prod_{\mu=1}^{r}\left(\rho \cdot \alpha^{\mu}+\sigma \cdot \beta^{\mu}+\tau \cdot \gamma^{\mu}-k\right) !\right\}(\rho \cdot \widetilde{\alpha}+\sigma \cdot \widetilde{\beta}+\tau \cdot \widetilde{\gamma}-k) !}{\left\{\rho \cdot\left(\alpha+\bar{\alpha}+\widetilde{\alpha}-\alpha^{\prime}\right)+\sigma \cdot\left(\beta+\bar{\beta}+\widetilde{\beta}-\beta^{\prime}\right)+\tau \cdot\left(\gamma+\bar{\gamma}+\widetilde{\gamma}-\gamma^{\prime}\right)-k\right\} !} \\
\leq & \mathcal{C}^{r-|\alpha|-|\beta|-|\gamma|+1} \frac{\left\{\prod_{\mu=1}^{r}\left(\left|\alpha^{\mu}\right|+\left|\beta^{\mu}\right|+\left|\gamma^{\mu}\right|\right) !\right\}(|\widetilde{\alpha}|+|\widetilde{\beta}|+|\widetilde{\gamma}|) !}{\left(\left|\alpha+\bar{\alpha}+\widetilde{\alpha}-\alpha^{\prime}\right|+\left|\beta+\bar{\beta}+\widetilde{\beta}-\beta^{\prime}\right|+\left|\gamma+\bar{\gamma}+\widetilde{\gamma}-\gamma^{\prime}\right|\right) !} \\
& \left(\left|\alpha^{1}\right|+\left|\beta^{1}\right|+\left|\gamma^{1}\right| \geq 1, \ldots,\left|\alpha^{r}\right|+\left|\beta^{r}\right|+\left|\gamma^{r}\right| \geq 1, \widetilde{\alpha} \geq \alpha^{\prime}, \widetilde{\beta} \geq \beta^{\prime}, \widetilde{\gamma} \geq \gamma^{\prime}\right) .
\end{aligned}
$$

We remark that $|\widetilde{\alpha}|+|\widetilde{\beta}|+|\widetilde{\gamma}|-1 \geq 0$ since $|\widetilde{\alpha}| \geq\left|\alpha^{\prime}\right| \geq 1$.

Let us prove (6.18). $r k \geq(1-\rho) \cdot \alpha+(1-\sigma) \cdot \beta+(1-\tau) \cdot \gamma+\rho \cdot \alpha^{\prime}+\sigma \cdot \beta^{\prime}+\tau \cdot \gamma^{\prime}-1$ implies

$$
\begin{aligned}
& \frac{\left\{\prod_{\mu=1}^{r}\left(\rho \cdot \alpha^{\mu}+\sigma \cdot \beta^{\mu}+\tau \cdot \gamma^{\mu}-k\right) !\right\}(\rho \cdot \widetilde{\alpha}+\sigma \cdot \widetilde{\beta}+\tau \cdot \widetilde{\gamma}-k) !}{\left\{\rho \cdot\left(\alpha+\bar{\alpha}+\widetilde{\alpha}-\alpha^{\prime}\right)+\sigma \cdot\left(\beta+\bar{\beta}+\widetilde{\beta}-\beta^{\prime}\right)+\tau \cdot\left(\gamma+\bar{\gamma}+\widetilde{\gamma}-\gamma^{\prime}\right)-k\right\} !} \\
& =\frac{\left\{\prod_{\mu=1}^{r} \Gamma\left(\rho \cdot \alpha^{\mu}+\sigma \cdot \beta^{\mu}+\tau \cdot \gamma^{\mu}-k+1\right)\right\} \Gamma(\rho \cdot \widetilde{\alpha}+\sigma \cdot \widetilde{\beta}+\tau \cdot \widetilde{\gamma}-k+1)}{\Gamma\left(\rho \cdot\left(\alpha+\bar{\alpha}+\widetilde{\alpha}-\alpha^{\prime}\right)+\sigma \cdot\left(\beta+\bar{\beta}+\widetilde{\beta}-\beta^{\prime}\right)+\tau \cdot\left(\gamma+\bar{\gamma}+\widetilde{\gamma}-\gamma^{\prime}\right)-k+1\right)} \\
& \leq \frac{\left\{\prod_{\mu=1}^{r} \Gamma\left(\rho \cdot \alpha^{\mu}+\sigma \cdot \beta^{\mu}+\tau \cdot \gamma^{\mu}-k+1\right)\right\} \Gamma(\rho \cdot \widetilde{\alpha}+\sigma \cdot \widetilde{\beta}+\tau \cdot \widetilde{\gamma}-k+1)}{\Gamma(K+|\alpha|+|\beta|+|\gamma|)} \\
& \equiv \boldsymbol{\Gamma},
\end{aligned}
$$

where

$$
K=\rho \cdot(\bar{\alpha}+\widetilde{\alpha})+\sigma \cdot(\bar{\beta}+\widetilde{\beta})+\tau \cdot(\bar{\gamma}+\widetilde{\gamma})-(r+1) k .
$$

The case $|\alpha|+|\beta|+|\gamma| \geq r+2$ : In this case it holds that

$$
\Gamma(K+|\alpha|+|\beta|+|\gamma|)=\Gamma(K+r+1) \prod_{l=r+1}^{|\alpha|+|\beta|+|\gamma|-1}(K+l),
$$

which implies

$$
\begin{aligned}
\boldsymbol{\Gamma}= & \frac{\left\{\prod_{\mu=1}^{r} \Gamma\left(\rho \cdot \alpha^{\mu}+\sigma \cdot \beta^{\mu}+\tau \cdot \gamma^{\mu}-k+1\right)\right\} \Gamma(\rho \cdot \widetilde{\alpha}+\sigma \cdot \widetilde{\beta}+\tau \cdot \widetilde{\gamma}-k+1)}{\Gamma(K+r+1)} \\
& \times \frac{1}{\prod_{l=r+1}^{|\alpha|+|\beta|+|\gamma|-1}(K+l)} \\
\leq & \frac{\left\{\prod_{\mu=1}^{r}\left(\left|\alpha^{\mu}\right|+\left|\beta^{\mu}\right|+\left|\gamma^{\mu}\right|\right) !\right\}(|\widetilde{\alpha}|+|\widetilde{\beta}|+|\widetilde{\gamma}|) !}{(\widetilde{K}+r) !} \frac{1}{\prod_{l=r+1}^{|\alpha|+|\beta|+|\gamma|-1}(K+l)} \\
= & \frac{\left\{\prod_{\mu=1}^{r}\left(\left|\alpha^{\mu}\right|+\left|\beta^{\mu}\right|+\left|\gamma^{\mu}\right|\right) !\right\}(|\widetilde{\alpha}|+|\widetilde{\beta}|+|\widetilde{\gamma}|) !}{\widetilde{K} !}
\end{aligned}
$$




$$
\times \frac{1}{\prod_{l=1}^{r}(\widetilde{K}+l)} \frac{1}{\prod_{l=r+1}^{|\alpha|+|\beta|+|\gamma|-1}(K+l)},
$$

where

$$
\widetilde{K}=|\bar{\alpha}+\widetilde{\alpha}|+|\bar{\beta}+\widetilde{\beta}|+|\bar{\gamma}+\widetilde{\gamma}| .
$$

Moreover it follows from $K \geq \widetilde{K}$ that

$$
\begin{aligned}
& \frac{(|\widetilde{\alpha}|+|\widetilde{\beta}|+|\widetilde{\gamma}|-1) !}{\left(|\widetilde{\alpha}|+|\widetilde{\beta}|+|\widetilde{\gamma}|-\left|\alpha^{\prime}\right|-\left|\beta^{\prime}\right|-\left|\gamma^{\prime}\right|\right) !} \frac{\left(\widetilde{K}+\left|\alpha-\alpha^{\prime}\right|+\left|\beta-\beta^{\prime}\right|+\left|\gamma-\gamma^{\prime}\right|\right) !}{\widetilde{K} !} \\
& \quad \times \frac{1}{\prod_{l=1}^{r}(\widetilde{K}+l)} \frac{1}{\prod_{l=r+1}^{|\alpha|+|\beta|+|\gamma|-1}(K+l)} \\
& \leq \frac{(|\widetilde{\alpha}|+|\widetilde{\beta}|+|\widetilde{\gamma}|-1) !}{\left(|\widetilde{\alpha}|+|\widetilde{\beta}|+|\widetilde{\gamma}|-\left|\alpha^{\prime}\right|-\left|\beta^{\prime}\right|-\left|\gamma^{\prime}\right|\right) !} \frac{\left(\widetilde{K}-\left|\alpha^{\prime}\right|-\left|\beta^{\prime}\right|-\left|\gamma^{\prime}\right|+1\right) !}{\widetilde{K} !} \\
& \quad \times \prod_{l=1}^{|\alpha|+|\beta|+|\gamma|-1} \frac{\widetilde{K}-\left|\alpha^{\prime}\right|-\left|\beta^{\prime}\right|-\left|\gamma^{\prime}\right|+1+l}{\widetilde{K}+l} \\
& \leq 1 .
\end{aligned}
$$

By the above inequalities we obtain (6.18). In the case $|\alpha|+|\beta|+|\gamma| \leq r+1$ also, we can prove (6.18) similarly.

(3): Let us estimate as

$$
\frac{\left|\alpha+\bar{\alpha}+\widetilde{\alpha}-\alpha^{\prime}\right|}{\left\{\prod_{\mu=1}^{r}\left|\lambda \cdot \alpha^{\mu}-f_{u}(0,0)\right|\right\}\left|\lambda \cdot \widetilde{\alpha}-f_{u}(0,0)\right|} \leq C_{r \alpha \alpha^{\prime}} .
$$

Then it is sufficient to prove the following inequality:

$$
\begin{aligned}
& \frac{\widetilde{\alpha} !}{\left(\widetilde{\alpha}-\alpha^{\prime}\right) !} \frac{\widetilde{\beta} !}{\left(\widetilde{\beta}-\beta^{\prime}\right) !} \frac{\widetilde{\gamma} !}{\left(\widetilde{\gamma}-\gamma^{\prime}\right) !} \\
& \quad \times \frac{\left\{\prod_{\mu=1}^{r}\left(\rho \cdot \alpha^{\mu}+\sigma \cdot \beta^{\mu}+\tau \cdot \gamma^{\mu}-k\right) !\right\}(\rho \cdot \widetilde{\alpha}+\sigma \cdot \widetilde{\beta}+\tau \cdot \widetilde{\gamma}-k) !}{\left\{\rho \cdot\left(\alpha+\bar{\alpha}+\widetilde{\alpha}-\alpha^{\prime}\right)+\sigma \cdot\left(\beta+\bar{\beta}+\widetilde{\beta}-\beta^{\prime}\right)+\tau \cdot\left(\gamma+\bar{\gamma}+\widetilde{\gamma}-\gamma^{\prime}\right)-k\right\} !} \\
& \leq \mathcal{C}^{r-|\alpha|-|\beta|-|\gamma|+1} \frac{\left\{\prod_{\mu=1}^{r}\left|\alpha^{\mu}\right| !\left(\left|\beta^{\mu}\right|+\left|\gamma^{\mu}\right|\right) !\right\}|\widetilde{\alpha}| !(|\widetilde{\beta}|+|\widetilde{\gamma}|) !}{\left(\left|\alpha+\bar{\alpha}+\widetilde{\alpha}-\alpha^{\prime}\right|-1\right) !\left(\left|\beta+\bar{\beta}+\widetilde{\beta}-\beta^{\prime}\right|+\left|\gamma+\bar{\gamma}+\widetilde{\gamma}-\gamma^{\prime}\right|\right) !} \\
& \quad\left(\left|\alpha^{1}\right|+\left|\beta^{1}\right|+\left|\gamma^{1}\right| \geq 1, \ldots,\left|\alpha^{r}\right|+\left|\beta^{r}\right|+\left|\gamma^{r}\right| \geq 1, \widetilde{\alpha} \geq \alpha^{\prime}, \widetilde{\beta} \geq \beta^{\prime}, \widetilde{\gamma} \geq \gamma^{\prime}\right) .
\end{aligned}
$$

We remark that $\left|\alpha+\bar{\alpha}+\widetilde{\alpha}-\alpha^{\prime}\right|-1 \geq 0$ since $|\alpha| \geq 1$.

Let us prove (6.20). The case $|\alpha|+|\beta|+|\gamma| \geq r+2$ : From (6.16) and the 
proof of (2) we see that

$$
\begin{aligned}
\boldsymbol{\Gamma} & \leq \frac{\left\{\prod_{\mu=1}^{r}\left(\left|\alpha^{\mu}\right|+\left|\beta^{\mu}\right|+\left|\gamma^{\mu}\right| !\right)\right\}(|\widetilde{\alpha}|+|\widetilde{\beta}|+|\widetilde{\gamma}|) !}{\widetilde{K} !} \frac{1}{\prod_{l=1}^{|\alpha|+|\beta|+|\gamma|-1}(\widetilde{K}+l)} \\
& \leq \frac{\left\{\prod_{\mu=1}^{r}\left|\alpha^{\mu}\right| !\left(\left|\beta^{\mu}\right|+\left|\gamma^{\mu}\right|\right) !\right\}|\widetilde{\alpha} !|(|\widetilde{\beta}|+|\widetilde{\gamma}|) !}{|\bar{\alpha}+\widetilde{\alpha}| !(|\bar{\beta}+\widetilde{\beta}|+|\bar{\gamma}+\widetilde{\gamma}|) !} \frac{1}{\prod_{l=1}^{|\alpha|+|\beta|+|\gamma|-1}(\widetilde{K}+l)} .
\end{aligned}
$$

Moreover it holds that

$$
\begin{aligned}
& \frac{\widetilde{\alpha} !}{\left(\widetilde{\alpha}-\alpha^{\prime}\right) !} \frac{\widetilde{\beta} !}{\left(\widetilde{\beta}-\beta^{\prime}\right) !} \frac{\widetilde{\gamma} !}{\left(\widetilde{\gamma}-\gamma^{\prime}\right) !} \\
& \times \frac{\left(\left|\alpha+\bar{\alpha}+\widetilde{\alpha}-\alpha^{\prime}\right|-1\right) !\left(\left|\beta+\bar{\beta}+\widetilde{\beta}-\beta^{\prime}\right|+\left|\gamma+\bar{\gamma}+\widetilde{\gamma}-\gamma^{\prime}\right|\right) !}{|\bar{\alpha}+\widetilde{\alpha}| !(|\bar{\beta}+\widetilde{\beta}|+|\bar{\gamma}+\widetilde{\gamma}|) !} \\
& \times \frac{1}{\prod_{l=1}^{|\alpha|+|\beta|+|\gamma|-1}(\widetilde{K}+l)} \\
= & \frac{\widetilde{\alpha} !}{\left(\widetilde{\alpha}-\alpha^{\prime}\right) !} \frac{\widetilde{\beta} !}{\left(\widetilde{\beta}-\beta^{\prime}\right) !} \frac{\widetilde{\gamma} !}{\left(\widetilde{\gamma}-\gamma^{\prime}\right) !} \frac{\left|\bar{\alpha}+\widetilde{\alpha}-\alpha^{\prime}\right| !\left(\left|\bar{\beta}+\widetilde{\beta}-\beta^{\prime}\right|+\left|\bar{\gamma}+\widetilde{\gamma}-\gamma^{\prime}\right|\right) !}{|\bar{\alpha}+\widetilde{\alpha}| !(|\bar{\beta}+\widetilde{\beta}|+|\bar{\gamma}+\widetilde{\gamma}|) !} \\
& \times\left\{\prod_{l=1}^{|\alpha|-1} \frac{\left|\bar{\alpha}+\widetilde{\alpha}-\alpha^{\prime}\right|+l}{\widetilde{K}+l}\right\} \cdot\left\{\prod_{l=1}^{|\beta|+|\gamma|} \frac{\left|\bar{\beta}+\widetilde{\beta}-\beta^{\prime}\right|+\left|\bar{\gamma}+\widetilde{\gamma}-\gamma^{\prime}\right|+l}{\widetilde{K}+|\alpha|-1+l}\right\}
\end{aligned}
$$

$\leq 1$

By (6.19), (6.21) and (6.22) we obtain (6.20). In the case $|\alpha|+|\beta|+|\gamma| \leq r+1$ also, we can prove (6.20) similarly.

The proof is completed.

From Lemma 6.1 , similarly to the proof of Lemma 4.3 we can prove the following lemma.

Lemma 6.2. Let $\mathcal{F}(x, y, z, u)$ be holomorphic at $(x, y, z, u)=(0,0,0,0)$. Let us put $\mathcal{F}_{r \alpha \beta \gamma \alpha^{\prime} \beta^{\prime} \gamma^{\prime}}(x, y, z, u)=\mathcal{F}(x, y, z, u) x^{\alpha} y^{\beta} z^{\gamma} u^{r} D_{x}{ }^{\alpha^{\prime}} D_{y}{ }^{\beta^{\prime}} D_{z}{ }^{\gamma^{\prime}} u$.

(1) Let $|\alpha|+|\beta|+|\gamma|+r \geq 1,\left|\alpha^{\prime}\right|+\left|\beta^{\prime}\right|+\left|\gamma^{\prime}\right| \geq 1$, and let us assume that $(\rho, \sigma, \tau) \in[1,+\infty)^{d}$ and $k$ satisfy (6.6) and (6.7), respectively. If $U, V \in$ 
$\widetilde{G}_{0, k}^{\{\rho,(\sigma, \tau)\}}(X,(Y, Z))\left(\right.$ resp. $\left.G_{0, k}^{\{\rho, \sigma, \tau\}}(X, Y, Z)\right)$, then it holds that

$$
\left.\begin{array}{c}
\left\|\mid \mathcal{F}_{r \alpha \beta \gamma \alpha^{\prime} \beta^{\prime} \gamma^{\prime}}\left(x, y, z, \Lambda^{-1} U\right)-\mathcal{F}_{r \alpha \beta \gamma \alpha^{\prime} \beta^{\prime} \gamma^{\prime}}\left(x, y, z, \Lambda^{-1} V\right)\right\|_{k, X,(Y, Z)}^{\{\rho,(\sigma, \tau)\}} \\
\leq C^{r+1} \mathcal{C}^{r-|\alpha|-|\beta|-|\gamma|} \frac{X^{\alpha}}{X^{\alpha^{\prime}}} \frac{Y^{\beta}}{Y^{\beta^{\prime}}} \frac{Z^{\gamma}}{Z^{\gamma^{\prime}}}\|U-V \mid\|_{k, X,(Y, Z)}^{\{\rho,(\sigma, \tau)\}} \\
\quad \times \mathfrak{F}\left(X, Y, Z,\left|\left\|U\left|\left\|_{k, X,(Y, Z)}^{\{\rho,(\sigma, \tau)\}},\right\|\right||V|\right\|_{k, X,(Y, Z)}^{\{\rho,(\sigma, \tau)\}} ; r\right)\right. \\
\left(\left\|\mathcal{F}_{r \alpha \beta \gamma \alpha^{\prime} \beta^{\prime} \gamma^{\prime}}\left(x, y, z, \Lambda^{-1} U\right)-\mathcal{F}_{r \alpha \beta \gamma \alpha^{\prime} \beta^{\prime} \gamma^{\prime}}\left(x, y, z, \Lambda^{-1} V\right)\right\|_{k, X, Y, Z}^{\{\rho, \sigma, \tau\}}\right. \\
\quad \leq \quad C^{r+1} \mathcal{C}^{r-|\alpha|-|\beta|-|\gamma|} \frac{X^{\alpha}}{X^{\alpha^{\prime}}} \frac{Y^{\beta}}{Y^{\beta^{\prime}}} \frac{Z^{\gamma}}{Z^{\gamma^{\prime}}}\|U-V\|_{k, X, Y, Z}^{\{\rho, \sigma, \tau\}} \\
\quad \times \mathfrak{F}\left(X, Y, Z,\|U\|_{k, X, Y, Z}^{\{\rho, \sigma, \tau\}},\|V\|_{k, X, Y, Z}^{\{\rho, \sigma, \tau\}} ; r\right)
\end{array}\right),
$$

where $C$ and $\mathcal{C}$ are same as in (6.4) and (6.9), respectively, and

$$
\begin{aligned}
\mathfrak{F}(X, Y, Z, U, V ; r)= & C \widetilde{\mathrm{S}}\left|\mathcal{F}_{u}\right|(X, Y, Z, \widetilde{\mathrm{S}} C(U+V)) \cdot U^{r+1} \\
& +|\mathcal{F}|(X, Y, Z, \widetilde{\mathrm{S}} C V) \cdot \sum_{\nu=0}^{r} U^{r-\nu} V^{\nu}
\end{aligned}
$$

$\left(\widetilde{\mathrm{S}}=\max \left\{\rho_{1}, \ldots, \rho_{m}, \sigma_{1}, \ldots, \sigma_{n}, \tau_{1}, \ldots, \tau_{p}\right\}\right)$.

(2) Let $|\alpha|+|\beta|+|\gamma|+r \geq 1$ and $\left|\alpha^{\prime}\right| \geq 1$, and let us assume that $(\rho, \sigma, \tau) \in[1,+\infty)^{d}$ and $k$ satisfy (6.10) and (6.11), respectively. If $U, V \in$ $G_{0, k}^{\{\rho, \sigma, \tau\}}(X, Y, Z)$, then it holds that

$$
\begin{aligned}
& \left\|\mathcal{F}_{r \alpha \beta \gamma \alpha^{\prime} \beta^{\prime} \gamma^{\prime}}\left(x, y, z, \Lambda^{-1} U\right)-\mathcal{F}_{r \alpha \beta \gamma \alpha^{\prime} \beta^{\prime} \gamma^{\prime}}\left(x, y, z, \Lambda^{-1} V\right)\right\|_{k, X, Y, Z}^{\{\rho, \sigma, \tau\}} \\
& \leq C^{r+1} \widetilde{\mathcal{C}}^{r-|\alpha|-|\beta|-|\gamma|+1} \frac{X^{\alpha}}{X^{\alpha^{\prime}}} \frac{Y^{\beta}}{Y^{\beta^{\prime}}} \frac{Z^{\gamma}}{Z^{\gamma^{\prime}}}\|U-V\|_{k, X, Y, Z}^{\{\rho, \sigma, \tau\}} \\
& \quad \times \mathfrak{F}\left(X, Y, Z,\|U\|_{k, X, Y, Z}^{\{\rho, \sigma\}},\|V\|_{k, X, Y, Z}^{\{\rho, \sigma, \tau\}} ; r\right),
\end{aligned}
$$

where $\widetilde{\mathcal{C}}$ is same as in (6.13).

(3) Let $|\alpha| \geq 1$ and $\left|\alpha^{\prime}\right|+\left|\beta^{\prime}\right|+\left|\gamma^{\prime}\right| \geq 1$. Let us assume that $(\rho, \sigma, \tau) \in$ $[1,+\infty)^{d}$ and $k$ satisfy (6.10) and (6.11), respectively. If $U, V \in \widetilde{G}_{0, k}^{\{\rho,(\sigma, \tau)\}}(X$, $(Y, Z))$, it holds that

$$
\begin{aligned}
& \left\|\mathcal{F}_{r \alpha \beta \gamma \alpha^{\prime} \beta^{\prime} \gamma^{\prime}}\left(x, y, z, \Lambda^{-1} U\right)-\mathcal{F}_{r \alpha \beta \gamma \alpha^{\prime} \beta^{\prime} \gamma^{\prime}}\left(x, y, z, \Lambda^{-1} V\right)\right\|_{k, X,(Y, Z)}^{\{\rho,(\sigma, \tau)\}} \\
& \leq C_{r \alpha \alpha^{\prime}} \widetilde{\mathcal{C}}^{r-|\alpha|-|\beta|-|\gamma|+1} \frac{X^{\alpha}}{X^{\alpha^{\prime}}} \frac{Y^{\beta}}{Y^{\beta^{\prime}}} \frac{Z^{\gamma}}{Z^{\gamma^{\prime}}}|| U-V \mid \|_{k, X,(Y, Z)}^{\{\rho,(\sigma, \tau)\}} \\
& \times \mathfrak{F}\left(X, Y, Z,|||U|\left\|_{k, X,(Y, Z)}^{\{\rho,(\sigma, \tau)\}},\right\||| V \mid \|_{k, X,(Y, Z)}^{\{\rho,(\sigma, \tau)\}} ; r\right) .
\end{aligned}
$$


Proof of Case (i) of Theorem 2.1 (continued). Put $k_{0}=\min \left\{\rho_{1}-1, \ldots\right.$, $\left.\rho_{m}-1, \sigma_{1}-1, \ldots, \sigma_{n}-1, \tau_{1}-1, \ldots, \tau_{p}-1\right\}$.

Case $(\mathrm{I}) ; \quad P^{\prime \prime \prime \prime \prime}=0$ : Let $U(x, y, z) \in \widetilde{G}_{0, k_{0}}^{\{\rho,(\sigma, \tau)\}}(X,(Y, Z))$. Then it follows from the assumption $(\rho, \sigma, \tau) \in \widetilde{S}_{1} \cap S_{1} \cap \widetilde{S}_{1}^{\prime}$ and Lemmas 3.2, (2)', 3.4, (2), 3.5, (2), 6.1, (1), (3) that $T U(x, y, z) \in \widetilde{G}_{0, k_{0}}^{\{\rho,(\sigma)\}}(X,(Y, Z))$ and that

$$
\begin{aligned}
\||| T U \mid\| \leq & A_{1}\left(X, Y, Z,\||U \||)+A_{2}\left(X, Y, Z,\left\||\|U \mid\|)+A_{3}(X, Y, Z,\||U \||)\right.\right.\right. \\
& +B(X, Y, Z,\||U \||)
\end{aligned}
$$

where

$$
\begin{aligned}
& A_{1}(X, Y, Z, U)=C \sum_{i=1}^{m-1} \frac{X_{i+1}}{X_{i}} U \\
& +\sum_{i=1}^{m} \frac{1}{X_{i}}\left(\sum_{\substack{|\alpha|+|\beta|+|\gamma|+r \geq 2 \\
|\alpha| \geq 1}}^{\text {finite }}\left|c_{i \alpha \beta \gamma r}\right|(X, Y, Z, \widetilde{\mathrm{S}} C U)\right. \\
& \left.\times C_{r \alpha \mathrm{e}_{i}^{(m)}} \widetilde{\mathcal{C}}^{r-|\alpha|-|\beta|-|\gamma|+1} X^{\alpha} Y^{\beta} Z^{\gamma} U^{r+1}\right), \\
& A_{2}(X, Y, Z, U) \\
& =\sum_{j=1}^{n} \frac{1}{Y_{j}}\left(\sum_{\substack{|\alpha|+|\beta|+|\gamma|+r \geq 2 \\
|\alpha| \geq 1}}^{\text {finite }}\left|d_{j \alpha \beta \gamma r}\right|(X, Y, Z, \widetilde{\mathrm{S}} C U)\right. \\
& \left.\times C_{r \alpha 0} \widetilde{\mathcal{C}}^{r-|\alpha|-|\beta|-|\gamma|+1} X^{\alpha} Y^{\beta} Z^{\gamma} U^{r+1}\right) \\
& +\sum_{q=1}^{p} \frac{1}{Z_{q}}\left(\sum_{\substack{|\alpha|+|\beta|+|\gamma|+r \geq 2 \\
|\alpha| \geq 1}}^{\text {finite }}\left|e_{q \alpha \beta \gamma r}\right|(X, Y, Z, \widetilde{\mathrm{S}} C U)\right. \\
& \left.\times C_{r \alpha 0} \widetilde{\mathcal{C}}^{r-|\alpha|-|\beta|-|\gamma|+1} X^{\alpha} Y^{\beta} Z^{\gamma} U^{r+1}\right), \\
& A_{3}(X, Y, Z, U)=C \sum_{j=1}^{n-1} \frac{Y_{j+1}}{Y_{j}} U \\
& +\sum_{j=1}^{n} \frac{1}{Y_{j}}\left(\sum_{|\beta|+|\gamma|+r \geq 2}^{\text {finite }}\left|d_{j \beta \gamma r}\right|(X, Y, Z, \widetilde{\mathrm{S}} C U) \mathcal{C}^{r-|\beta|-|\gamma|} Y^{\beta} Z^{\gamma}(C U)^{r+1}\right)
\end{aligned}
$$




$$
\begin{aligned}
& +\sum_{q=1}^{p} \frac{1}{Z_{q}}\left(\sum_{|\beta|+|\gamma|+r \geq 2}^{\text {finite }}\left|e_{q \beta \gamma r}\right|(X, Y, Z, \widetilde{\mathrm{S}} C U) \mathcal{C}^{r-|\beta|-|\gamma|} Y^{\beta} Z^{\gamma}(C U)^{r+1}\right) \\
& B(X, Y, Z, U)=\left|g_{0}\right|(X, Y, Z)+\frac{1}{\widetilde{\mathrm{S}}}|g|(X, Y, Z, \widetilde{\mathrm{S}} C U) .
\end{aligned}
$$

For simplicity, we write $\||\cdot|\|_{k_{0}, X,(Y, Z)}^{\{\rho,(\sigma, \tau)\}}$ as $\|\cdot|\||$. We can take $X, Y, Z$ and $\varepsilon$ such that $A_{1}(X, Y, Z, \varepsilon)+A_{2}(X, Y, Z, \varepsilon)+A_{3}(X, Y, Z, \varepsilon)+B(X, Y, Z, \varepsilon) \leq \varepsilon$. This shows that the operator $T: \widetilde{G}_{0, k_{0}}^{\{\rho,(\sigma, \tau)\}}(X,(Y, Z) ; \varepsilon) \rightarrow \widetilde{G}_{0, k_{0}}^{\{\rho,(\sigma, \tau)\}}(X,(Y, Z) ; \varepsilon)$ is well-defined.

Moreover it follows from Lemmas 3.6, (2), 6.2, (1), (3) that

$$
\begin{aligned}
& \left\||| T U-T V|\| \leq||| U-V|| \mid \times\left\{\widetilde{A}_{1}(X, Y, Z, \||U|||,|| V|| \mid)\right.\right. \\
& +\widetilde{A}_{2}(X, Y, Z,\|U\||\|,\| V \|| \mid) \\
& \left.+\widetilde{A}_{3}(X, Y, Z, \||| U|||,||| V|| \mid)+\widetilde{B}(X, Y, Z,|||U| \|,|||V|||)\right\},
\end{aligned}
$$

where

$$
\begin{aligned}
& \widetilde{A}_{1}(X, Y, Z, U, V)=C \sum_{i=1}^{m-1} \frac{X_{i+1}}{X_{i}} \\
& +\widetilde{\mathrm{S}} C \sum_{i=1}^{m} \frac{1}{X_{i}}\left(\sum_{|\alpha|+|\beta|+|\gamma|+r \geq 2,|\alpha| \geq 1}^{\text {finite }}\left|c_{i \alpha \beta \gamma r, u}\right|(X, Y, Z, \widetilde{\mathrm{S}} C(U+V))\right. \\
& \left.\times C_{r \alpha \mathrm{e}_{i}^{(m)}} \widetilde{\mathcal{C}}^{r-|\alpha|-|\beta|-|\gamma|+1} X^{\alpha} Y^{\beta} Z^{\gamma} U^{r+1}\right) \\
& +\sum_{i=1}^{m} \frac{1}{X_{i}}\left(\sum_{|\alpha|+|\beta|+|\gamma|+r \geq 2,|\alpha| \geq 1}^{\text {finite }}\left|c_{i \alpha \beta \gamma r}\right|(X, Y, Z, \widetilde{\mathrm{S}} C V)\right. \\
& \left.\times C_{r \alpha \mathrm{e}_{i}^{(m)}} \widetilde{\mathcal{C}}^{r-|\alpha|-|\beta|-|\gamma|+1} X^{\alpha} Y^{\beta} Z^{\gamma} \sum_{\nu=0}^{r} U^{r-\nu} V^{\nu}\right), \\
& \widetilde{A}_{2}(X, Y, Z, U, V) \\
& =\widetilde{\mathrm{S}} C \sum_{j=1}^{n} \frac{1}{Y_{j}}\left(\sum_{|\alpha|+|\beta|+|\gamma|+r \geq 2,|\alpha| \geq 1}^{\text {finite }}\left|d_{j \alpha \beta \gamma r, u}\right|(X, Y, Z, \widetilde{\mathrm{S}} C(U+V))\right. \\
& \left.\times C_{r \alpha 0} \widetilde{\mathcal{C}}^{r-|\alpha|-|\beta|-|\gamma|+1} X^{\alpha} Y^{\beta} Z^{\gamma} U^{r+1}\right) \\
& +\sum_{j=1}^{n} \frac{1}{Y_{j}}\left(\sum_{|\alpha|+|\beta|+|\gamma|+r \geq 2,|\alpha| \geq 1}^{\text {finite }}\left|d_{j \alpha \beta \gamma}\right|(X, Y, Z, \widetilde{\mathrm{S}} C V)\right.
\end{aligned}
$$




$$
\begin{aligned}
& \left.\times C_{r \alpha 0} \widetilde{\mathcal{C}}^{r-|\alpha|-|\beta|-|\gamma|+1} X^{\alpha} Y^{\beta} Z^{\gamma} \sum_{\nu=0}^{r} U^{r-\nu} V^{\nu}\right) \\
& +\widetilde{\mathrm{S}} C \sum_{q=1}^{p} \frac{1}{Z_{q}}\left(\sum_{|\alpha|+|\beta|+|\gamma|+r \geq 2,|\alpha| \geq 1}^{\text {finite }}\left|e_{q \alpha \beta \gamma r, u}\right|(X, Y, Z, \widetilde{\mathrm{S}} C(U+V))\right. \\
& \left.\times C_{r \alpha 0} \widetilde{\mathcal{C}}^{r-|\alpha|-|\beta|-|\gamma|+1} X^{\alpha} Y^{\beta} Z^{\gamma} U^{r+1}\right) \\
& +\sum_{q=1}^{p} \frac{1}{Z_{q}}\left(\sum_{|\alpha|+|\beta|+|\gamma|+r \geq 2,|\alpha| \geq 1}^{\text {finite }}\left|e_{q \alpha \beta \gamma r}\right|(X, Y, Z, \widetilde{\mathrm{S}} C V)\right. \\
& \left.\times C_{r \alpha 0} \widetilde{C}^{r-|\alpha|-|\beta|-|\gamma|+1} X^{\alpha} Y^{\beta} Z^{\gamma} \sum_{\nu=0}^{r} U^{r-\nu} V^{\nu}\right), \\
& \widetilde{A}_{3}(X, Y, Z, U, V)=C \sum_{j=1}^{n-1} \frac{Y_{j+1}}{Y_{j}} \\
& +\widetilde{\mathrm{S}} C \sum_{j=1}^{n} \frac{1}{Y_{j}}\left(\sum_{|\beta|+|\gamma|+r \geq 2}^{\text {finite }}\left|d_{j \beta \gamma r, u}\right|(X, Y, Z, \widetilde{\mathrm{S}} C(U+V))\right. \\
& \left.\times \mathcal{C}^{r-|\beta|-|\gamma|} Y^{\beta} Z^{\gamma}(C U)^{r+1}\right) \\
& +\sum_{j=1}^{m} \frac{1}{Y_{j}}\left(\sum_{|\beta|+|\gamma|+r \geq 2}^{\text {finite }}\left|d_{j \beta \gamma r}\right|(X, Y, Z, \widetilde{\mathrm{S}} C V)\right. \\
& \left.\times \mathcal{C}^{r-|\beta-| \gamma \mid} Y^{\beta} Z^{\gamma} C^{r+1} \sum_{\nu=0}^{r} U^{r-\nu} V^{\nu}\right) \\
& +\widetilde{\mathrm{S}} C \sum_{q=1}^{p} \frac{1}{Z_{q}}\left(\sum_{|\beta|+|\gamma|+r \geq 2}^{\text {finite }}\left|e_{q \beta \gamma r, u}\right|(X, Y, Z, \widetilde{\mathrm{S}} C(U+V))\right. \\
& \left.\times \mathcal{C}^{r-|\beta|-|\gamma|} Y^{\beta} Z^{\gamma}(C U)^{r+1}\right) \\
& +\sum_{q=1}^{p} \frac{1}{Z_{q}}\left(\sum_{|\beta|+|\gamma|+r \geq 2}^{\text {finite }}\left|e_{q \beta \gamma r}\right|(X, Y, Z, \widetilde{\mathrm{S}} C V)\right. \\
& \left.\times \mathcal{C}^{r-|\beta|-|\gamma|} Y^{\beta} Z^{\gamma} C^{r+1} \sum_{\nu=0}^{r} U^{r-\nu} V^{\nu}\right)
\end{aligned}
$$




$$
\widetilde{B}(X, Y, Z, U, V)=C\left|g_{u}\right|(X, Y, Z, \widetilde{\mathrm{S}} C(U+V)) .
$$

We can take $X, Y, Z$ and $\varepsilon$ such that $\widetilde{A}_{1}(X, Y, Z, \varepsilon, \varepsilon)+\widetilde{A}_{2}(X, Y, Z, \varepsilon, \varepsilon)+$ $\widetilde{A}_{3}(X, Y, Z, \varepsilon, \varepsilon)+\widetilde{B}(X, Y, Z, \varepsilon, \varepsilon)<1$. This shows that $T: \widetilde{G}_{0, k_{0}}^{\{\rho,(\sigma)\}}(X,(Y$, $Z) ; \varepsilon) \rightarrow \widetilde{G}_{0, k_{0}}^{\{\rho,(\sigma)\}}(X,(Y, Z) ; \varepsilon)$ is a contraction mapping.

Case (II); $\quad P^{\prime \prime}=0$ : Let $U(x, y, z) \in G_{0, k_{0}}^{\{\rho, \tau\}}(X, Y, Z)$. Then it follows from the assumption $(\rho, \sigma, \tau) \in \widetilde{S}_{1} \cap S_{1} \cap \widetilde{S}_{1}^{\prime \prime}$ and Lemmas 3.2, (1)', 3.4, (1), $3.5,(1), 6.1,(1),(2)$ that $T U(x, y, z) \in G_{0, k_{0}}^{\{\rho, \sigma, \tau\}}(X, Y, Z)$ and that

$$
\begin{aligned}
\|T U\| \leq & A_{4}(X, Y, Z,\|U\|)+A_{3}(X, Y, Z,\|U\|) \\
& +A_{5}(X, Y, Z,\|U\|)+B(X, Y, Z,\|U\|),
\end{aligned}
$$

where

$$
\begin{aligned}
& A_{4}(X, Y, Z, U)=C \sum_{i=1}^{m-1} \frac{X_{i+1}}{X_{i}} U \\
& +\sum_{i=1}^{m} \frac{1}{X_{i}}\left(\sum_{\substack{|\alpha|+|\beta|+|\gamma|+r \geq 2 \\
|\alpha| \geq 1}}^{\text {finite }}\left|c_{i \alpha \beta \gamma r}\right|(X, Y, Z, \widetilde{\mathrm{S}} C U)\right. \\
& \left.\times \widetilde{\mathcal{C}}^{r-|\alpha|-|\beta|-|\gamma|+1} X^{\alpha} Y^{\beta} Z^{\gamma}(C U)^{r+1}\right), \\
& \left.A_{5}(X, Y, Z, U) \quad c_{i \beta \gamma r} \mid(X, Y, Z, \widetilde{\mathrm{S}} C U) \widetilde{\mathcal{C}}^{r-|\beta|-|\gamma|+1} Y^{\beta} Z^{\gamma}(C U)^{r+1}\right) .
\end{aligned}
$$

For simplicity, we write $\|\cdot\|_{k_{0}, X, Y, Z}^{\{\rho, \sigma, \tau\}}$ as $\|\cdot\|$. We can take $X, Y, Z$ and $\varepsilon$ such that $A_{4}(X, Y, Z, \varepsilon)+A_{3}(X, Y, Z, \varepsilon)+A_{5}(X, Y, Z, \varepsilon)+B(X, Y, Z, \varepsilon) \leq \varepsilon$. This shows that the operator $T: G_{0, k_{0}}^{\{\rho, \sigma, \tau\}}(X, Y, Z ; \varepsilon) \rightarrow G_{0, k_{0}}^{\{\rho, \tau\}}(X, Y, Z ; \varepsilon)$ is well-defined.

Moreover it follows from Lemmas 3.6, (1), 6.2, (1), (2) that

$$
\begin{aligned}
\|T U-T V\| \leq & \|U-V\| \times\left\{\widetilde{A}_{4}(X, Y, Z,\|U\|,\|V\|)+\widetilde{A}_{3}(X, Y, Z,\|U\|,\|V\|)\right. \\
& \left.+\widetilde{A}_{5}(X, Y, Z,\|U\|,\|V\|)+\widetilde{B}(X, Y, Z,\|U\|,\|V\|)\right\}
\end{aligned}
$$

where

$$
\widetilde{A}_{4}(X, Y, Z, U, V)=C \sum_{i=1}^{m-1} \frac{X_{i+1}}{X_{i}}
$$




$$
\begin{aligned}
& +\widetilde{\mathrm{S}} C \sum_{i=1}^{m} \frac{1}{X_{i}}\left(\sum_{|\alpha|+|\beta|+|\gamma|+r \geq 2,|\alpha| \geq 1}^{\text {finite }}\left|c_{i \alpha \beta \gamma r, u}\right|(X, Y, Z, \widetilde{\mathrm{S}} C(U+V))\right. \\
& \left.\times \widetilde{\mathcal{C}}^{r-|\alpha|-|\beta|-|\gamma|+1} X^{\alpha} Y^{\beta} Z^{\gamma}(C U)^{r+1}\right) \\
& +\sum_{i=1}^{m} \frac{1}{X_{i}}\left(\sum_{|\alpha|+|\beta|+|\gamma|+r \geq 2,|\alpha| \geq 1}^{\text {finite }}\left|c_{i \alpha \beta \gamma r}\right|(X, Y, Z, \widetilde{\mathrm{S}} C V)\right. \\
& \left.\times \widetilde{\mathcal{C}}^{r-|\alpha|-|\beta|-|\gamma|+1} X^{\alpha} Y^{\beta} Z^{\gamma} C^{r+1} \sum_{\nu=0}^{r} U^{r-\nu} V^{\nu}\right), \\
& \widetilde{A}_{5}(X, Y, Z, U, V) \\
& =\widetilde{\mathrm{S}} C \sum_{i=1}^{m} \frac{1}{X_{i}}\left(\sum_{|\beta|+|\gamma|+r \geq 2}^{\text {finite }}\left|e_{q \beta \gamma r, u}\right|(X, Y, Z, \widetilde{\mathrm{S}} C(U+V))\right. \\
& \left.\times \widetilde{\mathcal{C}}^{r-|\beta|-|\gamma|+1} Y^{\beta} Z^{\gamma}(C U)^{r+1}\right) \\
& +\sum_{i=1}^{m} \frac{1}{X_{i}}\left(\sum_{|\beta|+|\gamma|+r \geq 2}^{\text {finite }}\left|e_{q \beta \gamma r}\right|(X, Y, Z, \widetilde{\mathrm{S}} C V)\right. \\
& \left.\times \widetilde{\mathcal{C}}^{r-|\beta|-|\gamma|+1} Y^{\beta} Z^{\gamma} C^{r+1} \sum_{\nu=0}^{r} U^{r-\nu} V^{\nu}\right) .
\end{aligned}
$$

We can take $X, Y, Z$ and $\varepsilon$ such that $\widetilde{A}_{4}(X, Y, Z, \varepsilon, \varepsilon)+\widetilde{A}_{3}(X, Y, Z, \varepsilon, \varepsilon)+$ $\widetilde{A}_{5}(X, Y, Z, \varepsilon, \varepsilon)+\widetilde{B}(X, Y, Z, \varepsilon, \varepsilon)<1$. This shows that $T: G_{0, k_{0}}^{\{\rho, \sigma\}}(X, Y, Z ; \varepsilon)$ $\rightarrow G_{0, k_{0}}^{\{\rho, \tau\}}(X, Y, Z ; \varepsilon)$ is a contraction mapping.

Case (III), (i); $\quad P^{\prime \prime} \neq 0, P^{\prime \prime \prime \prime} \neq 0$ and $(\rho, \sigma, \tau) \in \widetilde{S}_{1} \cap S_{1} \cap \widetilde{S}_{1}^{\prime} \cap S_{1}^{\prime \prime}:$ Let $U \in \widetilde{G}_{0, k_{0}}^{\{\rho,(\sigma, \tau)\}}(X,(Y, Z))$. Then it follows from the assumption $(\rho, \sigma, \tau) \in$ $\widetilde{S}_{1} \cap S_{1} \cap \widetilde{S}_{1}^{\prime} \cap S_{1}^{\prime \prime}$ and Lemmas 3.2, (2)', 3.4, (2), 3.5, (2), 6.1, (1), (3) that $T U(x, y, z) \in \widetilde{G}_{0, k_{0}}^{\{\rho,(\sigma)\}}(X,(Y, Z))$ and that

$$
\begin{aligned}
\||T U|\| \leq & A_{1}(X, Y, Z,\||U|\|)+A_{2}\left(X, Y, Z,\left\||\|U \mid\|)+A_{3}(X, Y, Z,\||U \||)\right.\right. \\
& +A_{6}(X, Y, Z,\||\|U \mid\|)+B(X, Y, Z,\|U\| \mid)
\end{aligned}
$$

where

$$
\begin{aligned}
& A_{6}(X, Y, Z, U) \\
& \quad=\sum_{i=1}^{m} \frac{1}{X_{i}}\left(\sum_{|\beta|+|\gamma|+r \geq 2}^{\text {finite }}\left|c_{i \beta \gamma r}\right|(X, Y, Z, \widetilde{\mathrm{S}} C U) \mathcal{C}^{r-|\beta|-|\gamma|} Y^{\beta} Z^{\gamma}(C U)^{r+1}\right) .
\end{aligned}
$$


Moreover it follows from Lemmas 3.6, (2), 6.2, (1), (3) that

$$
\begin{aligned}
& || T U-T V\left\||| \leq|| U-V|\|| \times\left\{\widetilde{A}_{1}(X, Y, Z,|||U|\|,|||V|\|)\right.\right. \\
& +\widetilde{A}_{2}(X, Y, Z,|||U|||,|||V|||)+\widetilde{A}_{3}(X, Y, Z,\||U|\|,|||V|||) \\
& \left.+\widetilde{A}_{6}(X, Y, Z, \||| U|||,||| V|| \mid)+\widetilde{B}(X, Y, Z,|||U| \|,|||V|||)\right\},
\end{aligned}
$$

where

$$
\begin{gathered}
\widetilde{A}_{6}(X, Y, Z, U, V) \\
=\widetilde{\mathrm{S}} C \sum_{i=1}^{m} \frac{1}{X_{i}}\left(\sum_{|\beta|+|\gamma|+r \geq 2}^{\text {finite }}\left|c_{i \beta \gamma r, u}\right|(X, Y, Z, \widetilde{\mathrm{S}} C(U+V))\right. \\
\left.\times \mathcal{C}^{r-|\beta|-|\gamma|} Y^{\beta} Z^{\gamma}(C U)^{r+1}\right) \\
+\sum_{i=1}^{m} \frac{1}{X_{i}}\left(\sum_{|\beta|+|\gamma|+r \geq 2}^{\text {finite }}\left|c_{i \beta \gamma r}\right|(X, Y, Z, \widetilde{\mathrm{S}} C V)\right. \\
\left.\times \mathcal{C}^{r-|\beta|-|\gamma|} Y^{\beta} Z^{\gamma} C^{r+1} \sum_{\nu=0}^{r} U^{r-\nu} V^{\nu}\right) .
\end{gathered}
$$

By the above expressions, similarly to the previous cases we can take $X$, $Y, Z$ and $\varepsilon$ such that $T: \widetilde{G}_{0, k_{0}}^{\{\rho,(\sigma, \tau)\}}(X,(Y, Z) ; \varepsilon) \rightarrow \widetilde{G}_{0, k_{0}}^{\{\rho,(\sigma, \tau)\}}(X,(Y, Z) ; \varepsilon)$ is well-defined and $T$ is a contraction mapping there.

Case (III), (ii); $\quad P^{\prime \prime} \neq 0, P^{\prime \prime \prime \prime \prime} \neq 0$ and $(\rho, \sigma, \tau) \in \widetilde{S}_{1} \cap S_{1} \cap S_{1}^{\prime} \cap \widetilde{S}_{1}^{\prime \prime}$ : Let $U \in G_{0, k_{0}}^{\{\rho, \sigma\}}(X, Y, Z)$. Then it follows from the assumption $(\rho, \sigma, \tau) \in$ $\widetilde{S}_{1} \cap S_{1} \cap S_{1}^{\prime} \cap \widetilde{S}_{1}^{\prime \prime}$ and Lemmas 3.2, (1)', 3.4, (1), 3.5, (1), 6.1, (1), (2) that $T U(x, y, z) \in G_{0, k_{0}}^{\{\rho, \sigma\}}(X, Y, Z)$ and that

$$
\begin{aligned}
\|T U\| \leq & A_{4}(X, Y, Z,\|U\|)+A_{7}(X, Y, Z,\|U\|)+A_{3}(X, Y, Z,\|U\|) \\
& +A_{5}(X, Y, Z,\|U\|)+B(X, Y, Z,\|U\|),
\end{aligned}
$$

where

$$
\begin{aligned}
& A_{7}(X, Y, Z, U) \\
& =\sum_{j=1}^{n} \frac{1}{Y_{j}}\left(\sum_{\substack{|\alpha|+|\beta|+|\gamma|+r \geq 2 \\
|\alpha| \geq 1}}^{\text {finite }}\left|d_{j \alpha \beta \gamma r}\right|(X, Y, Z, \widetilde{\mathrm{S}} C U)\right. \\
& \left.\times \mathcal{C}^{r-|\alpha|-|\beta|-|\gamma|} X^{\alpha} Y^{\beta} Z^{\gamma}(C U)^{r+1}\right)
\end{aligned}
$$




$$
\begin{aligned}
+\sum_{q=1}^{p} \frac{1}{Z_{q}}\left(\sum_{\substack{|\alpha|+|\beta|+|\gamma|+r \geq 1 \\
|\alpha| \geq 1}}^{\text {finite }} \mid\right. & e_{q \alpha \beta \gamma r} \mid(X, Y, Z, \widetilde{\mathrm{S}} C U) \\
& \left.\times \mathcal{C}^{r-|\alpha|-|\beta|-|\gamma|} X^{\alpha} Y^{\beta} Z^{\gamma}(C U)^{r+1}\right) .
\end{aligned}
$$

Moreover it follows from Lemmas 3.6, (1), 6.2, (1), (2) that

$$
\begin{aligned}
\|T U-T V\| \leq\|U-V\| \times\{ & \widetilde{A}_{4}(X, Y, Z,\|U\|,\|V\|)+\widetilde{A}_{7}(X, Y, Z,\|U\|,\|V\|) \\
& +\widetilde{A}_{3}(X, Y, Z,\|U\|,\|V\|)+\widetilde{A}_{5}(X, Y, Z,\|U\|,\|V\|) \\
& +\widetilde{B}(X, Y, Z,\|U\|,\|V\|)\}
\end{aligned}
$$

where

$$
\begin{aligned}
& \widetilde{A}_{7}(X, Y, Z, U, V) \\
& =\widetilde{\mathrm{S}} C \sum_{i=1}^{m} \frac{1}{Y_{j}}\left(\sum_{|\alpha|+|\beta|+|\gamma|+r \geq 2,|\alpha| \geq 1}^{\text {finite }}\left|d_{j \alpha \beta \gamma r, u}\right|(X, Y, Z, \widetilde{\mathrm{S}} C(U+V))\right. \\
& \left.\times \mathcal{C}^{r-|\alpha|-|\beta|-|\gamma|} X^{\alpha} Y^{\beta} Z^{\gamma}(C U)^{r+1}\right) \\
& +\sum_{i=1}^{m} \frac{1}{Y_{j}}\left(\sum_{|\alpha|+|\beta|+|\gamma|+r \geq 2,|\alpha| \geq 1}^{\text {finite }}\left|d_{j \alpha \beta \gamma r}\right|(X, Y, Z, \widetilde{\mathrm{S}} C V)\right. \\
& \left.\times \mathcal{C}^{r-|\alpha|-|\beta|-|\gamma|} X^{\alpha} Y^{\beta} Z^{\gamma} C^{r+1} \sum_{\nu=0}^{r} U^{r-\nu} V^{\nu}\right) \\
& +\widetilde{\mathrm{S}} C \sum_{q=1}^{p} \frac{1}{Z_{q}}\left(\sum_{|\alpha|+|\beta|+|\gamma|+r \geq 2,|\alpha| \geq 1}^{\text {finite }}\left|e_{q \alpha \beta \gamma r, u}\right|(X, Y, Z, \widetilde{\mathrm{S}} C(U+V))\right. \\
& \left.\times \mathcal{C}^{r-|\alpha|-|\beta|-|\gamma|} X^{\alpha} Y^{\beta} Z^{\gamma}(C U)^{r+1}\right) \\
& +\sum_{q=1}^{p} \frac{1}{Z_{q}}\left(\sum_{|\alpha|+|\beta|+|\gamma|+r \geq 2,|\alpha| \geq 1}^{\text {finite }}\left|e_{q \alpha \beta \gamma r}\right|(X, Y, Z, \widetilde{S} C V)\right. \\
& \left.\times \mathcal{C}^{r-|\alpha|-|\beta|-|\gamma|} X^{\alpha} Y^{\beta} Z^{\gamma} C^{r+1} \sum_{\nu=0}^{r} U^{r-\nu} V^{\nu}\right) .
\end{aligned}
$$


Therefore similarly to the previous cases we can take $X, Y, Z$ and $\varepsilon$ such that $T: G_{0, k_{0}}^{\{\rho, \sigma, \tau\}}(X, Y, Z ; \varepsilon) \rightarrow G_{0, k_{0}}^{\{\rho, \sigma\}}(X, Y, Z ; \varepsilon)$ is well-defined and $T$ is a contraction mapping there.

The proof is completed.

\section{References}

[1] Gérard, R. and Tahara, H., Singular nonlinear partial differential equations, Aspects of Mathematics, Friedr. Vieweg \& Sohn, Braunschweig, 1996.

[2] Hibino, M., Divergence property of formal solutions for singular first order linear partial differential equations, Publ. RIMS, Kyoto Univ., 35 (1999), 893-919.

[3] _ Formal Gevrey theory for singular first order semi-linear partial differential equations, Osaka J. Math., 41 (2004), 159-191.

[4] Kashiwara, M., Kawai, T. and Sjöstrand, J., On a class of linear partial differential equations whose formal solutions always converge, Ark. Mat., 17 (1979), 83-91.

[5] Miyake, M., Newton polygons and formal Gevrey indices in the Cauchy-Goursat-Fuchs type equations, J. Math. Soc. Japan, 43 (1991), 305-330.

[6] Miyake, M. and Hashimoto, Y., Newton polygons and Gevrey indices for linear partial differential operators, Nagoya Math. J., 128 (1992), 15-47.

[7] Miyake, M. and Shirai, A., Convergence of formal solutions of first order singular nonlinear partial differential equations in the complex domain, Dedicated to the memory of Bogdan Zieman, Ann. Polon. Math., 74 (2000), 215-228.

[8] Miyake, M. and Yoshino, M., Fredholm property of partial differential operators of irregular singular type, Ark. Mat., 33 (1995), 323-341.

[9] Ōshima, T., On the theorem of Cauchy-Kowalevsky for first order linear differential equations with degenerate principal symbols, Proc. Japan Acad., 49 (1973), 83-87.

[10] Ōuchi, S., Genuine solutions and formal solutions with Gevrey type estimates of nonlinear partial differential equations, J. Math. Sci. Univ. Tokyo, 2 (1995), 375-417.

[11] Sibuya, Y., Formal power series solutions in a parameter, J. Differential Equations, 190 (2003), 559-578.

[12] Yamazawa, H., Formal Gevrey class of formal power series solution for singular first order linear partial differential operators, Tokyo J. Math., 23 (2002), 537-561.

[13] L Newton polyhedrons and a formal Gevrey space of double indices for linear partial differential operators, Funkcial. Ekvac., 41 (1998), 337-345. 